\title{
Subcritical Water Extraction of Natural Products
}

\author{
Yan Cheng ${ }^{1,2,3}$, Fumin Xue ${ }^{1,2}$, Shuai Yu ${ }^{1,2}$, Shichao Du ${ }^{1,2}$ and Yu Yang ${ }^{3, *}$ \\ 1 School of Pharmaceutical Sciences, Qilu University of Technology (Former Shandong Academy of Sciences), \\ Jinan 250353, China; yancheng2020@qlu.edu.cn (Y.C.); xuefumin@qlu.edu.cn (F.X.); yushuai@qlu.edu.cn (S.Y.); \\ dushichao@tju.edu.cn (S.D.) \\ 2 Shandong Analysis and Test Centre, Qilu University of Technology (Former Shandong Academy of Sciences), \\ Jinan 250353, China \\ 3 Department of Chemistry, East Carolina University, Greenville, NC 27858, USA \\ * Correspondence: yangy@ecu.edu
}

Citation: Cheng, Y.; Xue, F.; Yu, S.; Du, S.; Yang, Y. Subcritical Water Extraction of Natural Products. Molecules 2021, 26, 4004. https:// doi.org/10.3390/molecules26134004

Academic Editors: Miguel Palma Lovillo, Ceferino A. Carrera Fernández and Ana Ruiz Rodríguez

Received: 10 May 2021

Accepted: 20 June 2021

Published: 30 June 2021

Publisher's Note: MDPI stays neutral with regard to jurisdictional claims in published maps and institutional affiliations.

Copyright: (c) 2021 by the authors. Licensee MDPI, Basel, Switzerland. This article is an open access article distributed under the terms and conditions of the Creative Commons Attribution (CC BY) license (https:// creativecommons.org/licenses/by/ $4.0 /)$.

\begin{abstract}
Subcritical water refers to high-temperature and high-pressure water. A unique and useful characteristic of subcritical water is that its polarity can be dramatically decreased with increasing temperature. Therefore, subcritical water can behave similar to methanol or ethanol. This makes subcritical water a green extraction fluid used for a variety of organic species. This review focuses on the subcritical water extraction (SBWE) of natural products. The extracted materials include medicinal and seasoning herbs, vegetables, fruits, food by-products, algae, shrubs, tea leaves, grains, and seeds. A wide range of natural products such as alkaloids, carbohydrates, essential oil, flavonoids, glycosides, lignans, organic acids, polyphenolics, quinones, steroids, and terpenes have been extracted using subcritical water. Various SBWE systems and their advantages and drawbacks have also been discussed in this review. In addition, we have reviewed co-solvents including ethanol, methanol, salts, and ionic liquids used to assist SBWE. Other extraction techniques such as microwave and sonication combined with SBWE are also covered in this review. It is very clear that temperature has the most significant effect on SBWE efficiency, and thus, it can be optimized. The optimal temperature ranges from 130 to $240{ }^{\circ} \mathrm{C}$ for extracting the natural products mentioned above. This review can help readers learn more about the SBWE technology, especially for readers with an interest in the field of green extraction of natural products. The major advantage of SBWE of natural products is that water is nontoxic, and therefore, it is more suitable for the extraction of herbs, vegetables, and fruits. Another advantage is that no liquid waste disposal is required after SBWE. Co Mpared with organic solvents, subcritical water not only has advantages in ecology, economy, and safety, but also its density, ion product, and dielectric constant can be adjusted by temperature. These tunable properties allow subcritical water to carry out class selective extractions such as extracting polar compounds at lower temperatures and less polar ingredients at higher temperatures. SBWE can mimic the traditional herbal decoction for preparing herbal medication and with higher extraction efficiency. Since SBWE employs high-temperature and high-pressure, great caution is needed for safe operation. Another challenge for application of SBWE is potential organic degradation under high temperature conditions. We highly recommend conducting analyte stability checks when carrying out SBWE. For analytes with poor SBWE efficiency, a small number of organic modifiers such as ethanol, surfactants, or ionic liquids may be added.
\end{abstract}

Keywords: natural products; subcritical water extraction; alkaloids; glycosides; flavonoids; essential oils; quinones; terpenes; lignans; organic acids; polyphenolics; steroids; carbohydrates

\section{Introduction}

For thousands of years, herbal medicine has played a vital role in treating diseases, especially in East Asia. The bioactive components in herbs and plants are the basis to prevention and treatment of many diseases [1]. Due to its relatively low side effects against chemically synthesized drugs, much attention is given to the extraction and separation 
of a wide range of bioactive compounds from herbs and plants. The 1000-year-old extraction process of the active pHarmaceutical ingredients (APIs) from medicinal herbs is the traditional herbal decoction (THD) method. However, there are some defects in THD, such as a long extraction time and decomposition of active pHarmaceutical ingredients. Methanol, ethanol, $n$-hexane, petroleum ether, diethyl ether, chloroform, ethyl acetate, and glycerol are often used as extraction solvents to increase the extraction efficiency and reduce extraction time. Obviously, these organic solvents are volatile, flammable, mostly toxic, and expensive. Thus, they are not safe extraction fluids for herbs, plants, fruits, vegetables, and the like.

Among the various new green extraction and separation technologies developed recently, SBWE is the most promising one. Subcritical water refers to the liquid water at temperature and pressure below its critical point $\left(\mathrm{Tc}=374.15^{\circ} \mathrm{C}, \mathrm{Pc}=22.1 \mathrm{Mpa}\right)$. The pressure of the subcritical water must be higher than the vapor pressure at a given temperature to keep water in the liquid state. With the increase of temperature, the pHysical-chemical properties of subcritical water change drastically. Its dielectric constant, viscosity, and surface tension all decrease steadily, and its diffusion coefficient is improved with increasing water temperature [2-5].

As shown in Figure 1 , at $27^{\circ} \mathrm{C}$ and $100 \mathrm{Mpa}$ water is a polar solvent, and its dielectric constant is 81.2 . Fortunately, when the temperature is raised to $350{ }^{\circ} \mathrm{C}$ at $100 \mathrm{Mpa}$, the dielectric constant of water decreases to around 20. In another words, water's polarity changes from strong polar to much less polar [2]. For example, the dielectric constant of water at $200{ }^{\circ} \mathrm{C}, 250{ }^{\circ} \mathrm{C}$, and $300{ }^{\circ} \mathrm{C}$ is equivalent to that of acetonitrile, methanol, and ethanol, respectively (Figure 1). Thus, even much less polar compounds can be extracted by subcritical water at high temperatures. Theoretically, based on its tunable polarity, subcritical water can extract many substances by adjusting extraction temperature and pressure conditions.

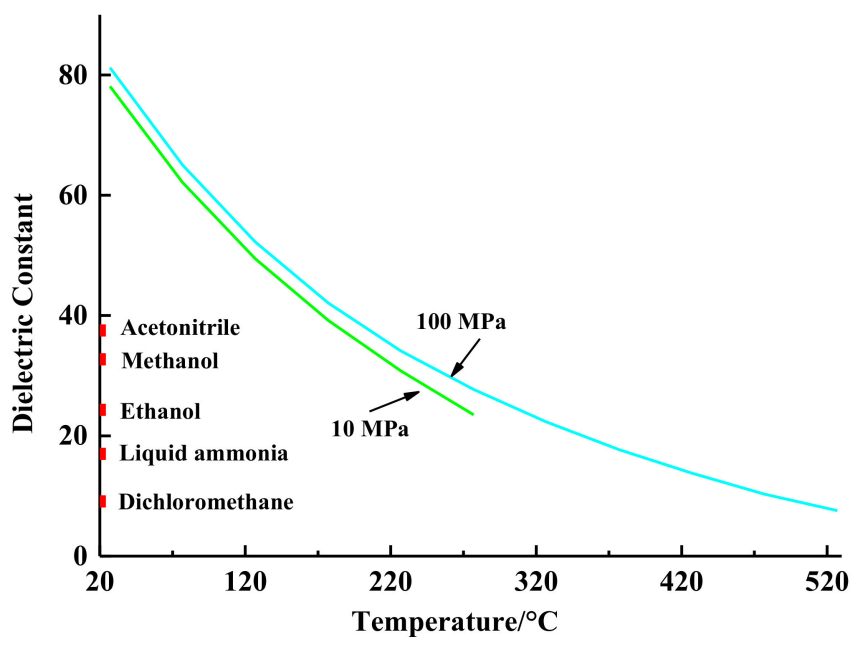

Figure 1. Dielectric constant of water at 27 to $527^{\circ} \mathrm{C}$ and 10 to $100 \mathrm{Mpa}$, acetonitrile, methanol, ethanol, liquid ammonia, and dichloromethane at $20^{\circ} \mathrm{C}$ and $0.1 \mathrm{Mpa}$.

In the actual extraction processes, since pressure has minimal influence on the dielectric constant of water, water's temperature is adjusted to control the dielectric constant of water in order to mimic various organic solvents. This unique characteristic of subcritical water allows water as the sole extraction fluid without any co-solvents such as acids, alkalis, catalysts, or organic solvents; this meets the principles of green chemical extraction since water is nontoxic.

Subcritical water applications can be found in the following areas: (1) reversed-phase liquid chromatography using subcritical water as the sole mobile pHase-subcritical water chromatography [3]; (2) extraction of environmental samples, such as the determination 
of organic pollutants in solid wastes, soils, sediments, and atmospheric particles [5,6]; (3) hydrolysis, degradation, polymerization, and synthesis reactions using subcritical water as both a solvent or a reactant [7]; (4) environmental remediation such as cleaning contaminated sewages and soils, decomposing pollutants (pesticides, polycyclic aromatic hydrocarbons, and polychlorinated biphenyls) and explosives [8]; (5) extraction of active ingredients from medicinal and seasoning herbs, vegetables, fruits, and other plant related matrices $[4,9]$.

This review focuses on SBWE of natural products in recent years. Principles, mechanisms, static and dynamic extraction modes, and factors affecting SBWE efficiency have been described by Gbashi and coworkers [10]. Since various research has focused on the extraction of flavonoids, carbohydrates, glycosides, organic acids, polyphenolics, alkaloids, essential oils, quinones, terpenes, lignans, and steroids [11,12], we provide a systematic and comprehensive overview on SBWE conditions, the function and activities of the active ingredients and the subcritical extracts, analysis methods, and co Mparison with other extraction methods for the above-mentioned natural products. Subcritical water can simultaneously extract several active ingredients from natural products. Separation, identification, and quantification of each natural product compound in the subcritical water extracts are achieved by liquid chromatography, gas chromatography, infrared spectrum, and/or mass spectrometry.

\section{Sample Matrices Extracted by Subcritical Water}

In general, the sample matrices extracted by subcritical water include the following four groups: Plants, food by-products, fungi, and marine algae. Based on the analysis and statistics of the references cited in this review, we generated Figure 2 to show the percentages of each type of sample matrices extracted by subcritical water. As shown in Figure 2, 62.3\% sample matrices extracted by subcritical water come from plants, followed by food by-products with $29.0 \%$, marine algae with $4.9 \%$, and fungi with $3.8 \%$.

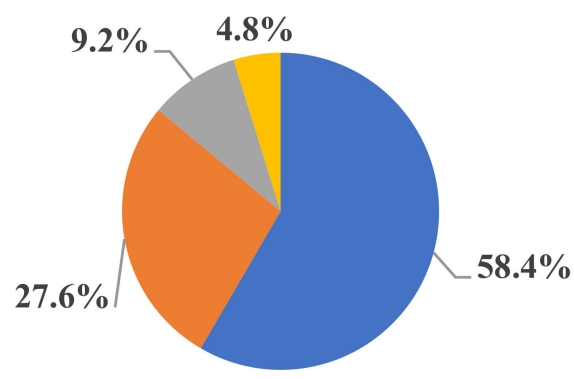

\section{$\backsim$ Plants $\backsim$ Food by-products $\square$ Marine algae $\square$ Fungi}

Figure 2. Types of sample matrices extracted by subcritical water.

For some medicinal herbs, their whole plants have pHarmaceutical values and are prescribed in traditional herbal medicine, while only part or parts of other medicinal herbs are used in herbal medicine. The parts of medicinal herbs include root, stems, leaves, flowers, seeds, and fruits. For example, the whole plants of Hedyotis diffusa Willd. [13] and Centella asiatica L. [14] have medicinal values and the leaves, nodes, petioles, and roots of these two herbs were grounded before SBWE. Stems and leaves of ginseng [15], root of Sophora flavescens Ait. [16] and Rheum tanguticum [17] and flowers of chamomile ligulate [18] were extracted using subcritical water. We carefully sorted out the medicinal herbs, and the percentage of each medicinal part is illustrated in Figure 3. One can see from Figure 3 the leaves of medicinal herbs that are most widely investigated in SBWE. Although the peels, hulls, brans, barks, shells, epicarps, pericarps, sorghums of fruits and seeds are the byproducts, they also contain many APIs. For example, onion, one of the most frequently consumed vegetables, is known to have many health benefits because of 
its flavonoid contents. Some results demonstrated that the onion peel extracts produced by SBWE technique have great potential as a source for useful antioxidants $[19,20]$. Grains, seeds, corn, and fruits have also been investigated using SBWE [21]. Among all papers reviewed, only one article reported that the wood of Aquilaria malaccensis has useful medicinal applications, and is used in traditional medicine to treat pain, fever, rheumatism, and asthma [22].

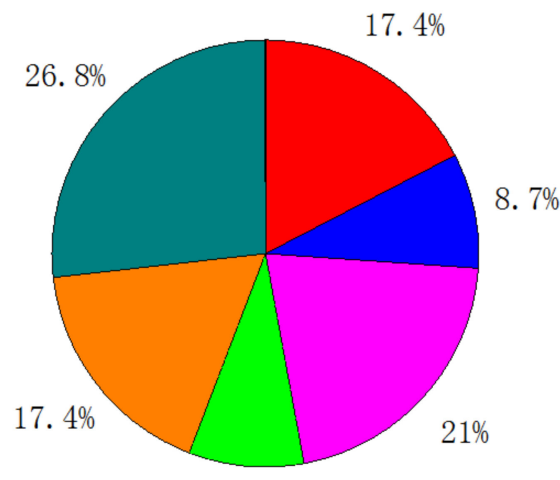

8. $7 \%$

Grain/Fruit/Seed $\square$ Stem $\square$ Leave $\square$ Flower/Stigmas

Root $\square$ Peel/Shell/Hull/Pomace/Bark/Dreg/Bran/Residue

Figure 3. Parts of medicinal herbs extracted by subcritical water.

\subsection{Plants}

Plants extracted by subcritical water mainly include herbs [15,23-29], vegetables with medicinal values [30,31], fruits [32-36], oilseed crops [37-40], shrubs, grains, tea leaves and beans [41-46]. These plants have been used in traditional medicine for thousands of years in some countries and have been proved to possess plentiful pHarmacologically active components. For example, ginseng is a valuable Chinese medicine, which has been used in China, Korea, Japan, and Brazil. Ginseng has been reported to contain a variety of bioactive chemical compounds including terpenes, polyacetylenes, alkaloids, vitamins, minerals, pHenolics, flavonoids, and triterpenes [15]. These active components possess antioxidant, anti-inflammatory, antidiabetic, antineoplastic, cardiovascular, immunoregulatory, and neuroregulatory activities [26]. Oregano is an herbaceous plant native to the Mediterranean regions $[47,48]$. Some healthy properties have been attributed to this plant, such as its powerful anti-bacteria and anti-fungal effects related to carvacrol and thymol compounds and some antioxidant activity.

\subsection{Food By-Products}

In essence, by-products are the wastes produced by fruit peels, tea filters, seed residues, vegetable peels, chestnut barks, cocoa shells, grain bran, lotus seed epicarp, and stems [49]. Many kinds of fruits have peels, such as bananas, oranges, and apples. Although we often throw them away, fruit peels may contain many APIs. Therefore, research on the by-products is worth doing. For example, orange peel is the main waste by-product of the juice extraction industry [50]. Nonetheless, orange peel is an attractive source of bioactive compounds, which include plenty of pHenolic and flavonoid compounds.

\subsection{Marine Algae}

Marine algae studied in SBWE include microalgae, seaweeds, and Haematococcus pluvialis [51-53]. Algae are rich in saturated fatty acid, monounsaturated fatty acid, and polyunsaturated fatty acid, which is healthy for the cardiovascular system. Omega-3, which plays a significant role in the body's inflammatory pathways and cell health, is 
especially used for cancer prevention and therapy. Using SBWE technology, algae could step up as one of the potential sources for future generation of omega-3.

\subsection{Fungi}

Some fungi are also responsible for some diseases in plants and animals, which also can be as vegetables, such as the edible-medicinal fungi, which include mushrooms (Lentinus edodes [54-57]; Grifola frondosa [58], Sagittaria sagittifolia L. [50-61]), and Cordyceps militaris (C. militaris) [62]. C. militaris is a type of precious edible-medicinal fungi widely distributed around the world. Extensive research demonstrated that extracts of $C$. militaris have multiple pHarmacological actions, such as anti-inflammation, improvement of insulin resistance, and antioxidant activity.

\section{SBWE Systems and Extraction Mechanism}

At the beginning, supercritical water $\left(\mathrm{T}>374{ }^{\circ} \mathrm{C}, \mathrm{P}>22.064 \mathrm{Mpa}\right)$ was widely involved in extraction of coal, oil sand bitumen [63,64], and oxidation [65]. Supercritical water requires harsh experimental conditions such as extremely high temperature and high pressure, which often bring severe corrosion on experimental equipment and connection tubing. Since 1990s, subcritical water has been gradually replacing supercritical water in the field of extraction because subcritical water requires relatively mild temperature and pressure. Thus, subcritical water has been extensively employed in the extraction of organic pollutants [66,67], and researchers have attempted to couple SBWE with liquid chromatography to reduce the analytical steps in the extraction and chromatographic analysis process [68-70]. To improve the extraction efficiency, ultrasonic-assisted SBWE [71] and microwave-assisted SBWE [72] have also been reported.

\subsection{Modes of SBWE}

There are mainly two SBWE modes, namely static extraction and dynamic extraction. Static extraction refers to an extraction method in which subcritical water and the sample to be extracted are added to an extraction vessel, as shown in Figure 4, and then heated to the desired temperature under a moderate pressure to keep water in the liquid state. After the set extraction time is reached, the extractant is collected for chromatographic analysis. This extraction process is similar to accelerated solvent extraction. The static extraction efficiency is normally lower than that of dynamic extraction.
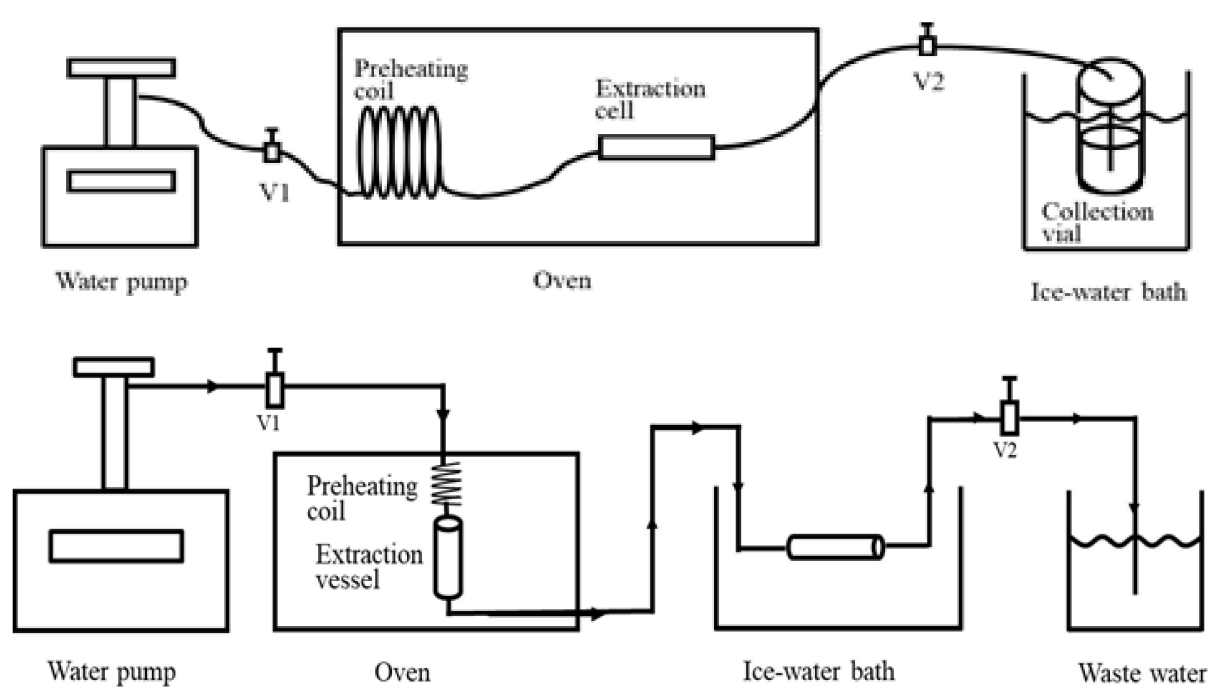

Figure 4. Subcritical water extraction system without solid trapping (top) and with solid trapping (bottom). 
Dynamic SBWE is a continuous extraction process, which means that after the samples are added to the extraction vessel, water is continuously fed into the extractor with a pump, and the extraction is carried out at a fixed temperature or in gradient temperature conditions. The pump can be set either under constant pressure mode or constant flow mode. Dynamic SBWE not only accelerates the mass transfer efficiency and shortens the extraction time, but also achieves selectively continuous extraction. However, dynamic SBWE may cause blockage to the SBWE system.

In many cases, both static and dynamic SBWE modes are used. SBWE operators normally conduct a period of static extraction, then followed by a dynamic extraction. Although a majority of the SBWE work is conducted without solid trapping of the extracted analytes (as shown in Figure 4, top), SBWE with a solid trap (Figure 4, bottom), which collects the extracted analytes can be easily coupled with liquid chromatography for analysis, as discussed further below.

\subsection{Online and Offline Coupling of SBWE with Liquid Chromatography}

SBWE system was coupled with high-performance liquid chromatography via solidphase trapping [69]. The offline coupling of SBWE with HPLC was reported [70]. The solid trap was pHysically removed from the SBWE system after each extraction and connected to an HPLC system for analysis. However, this step is eliminated in the online SBWE-HPLC approach. After SBWE, the analyte trap stays in place while the HPLC analysis is carried out by simply switching valves to connect the analyte trap with the HPLC unit.

Offline coupling of SBWE with subcritical water chromatography (SBWC) has also been reported [70]. SBWC refers to reversed-phase liquid chromatography using subcritical water as the sole mobile pHase component. As shown in Figure 5 (top), extracted analytes are collected in the sorbent trap during the SBWE step. After SBWE extraction, the sample trap is connected to a subcritical water chromatography system (Figure 5, bottom). The analytes collected in the sorbent trap during SBWE is thermally desorbed by heating the sample trap and then they are delivered into the SBWC system for analysis. The obvious advantage of SBWE-SBWC coupling is that organic solvents are eliminated in both extraction and chromatographic analysis steps. The challenge is, however, that the thermal desorption normally requires high temperature, which may potentially cause analyte degradation.

\subsection{Mechanisms of Subcritical Water Extraction}

The main SBWE extraction mechanism follows the "like dissolves like" rule. As mentioned in the Introduction section, the polarity of water can be tuned by temperature [2,3]. As shown in Figure 1, the dielectric constant of water is dramatically lowered (becoming less polar) with increasing temperature. This means that water's polarity is tunable by changing temperature. Because of this, polar compounds can be efficiently extracted by water at "lower" temperatures while less polar analytes require higher temperatures to achieve a reasonable extraction efficiency. Therefore, the less polar the analyte, the higher the temperature required. In addition, larger and more complex molecules require higher temperatures. Pressure does not have a significant effect on SBWE efficiency as long as the pressure is high enough to keep water in the liquid state. 

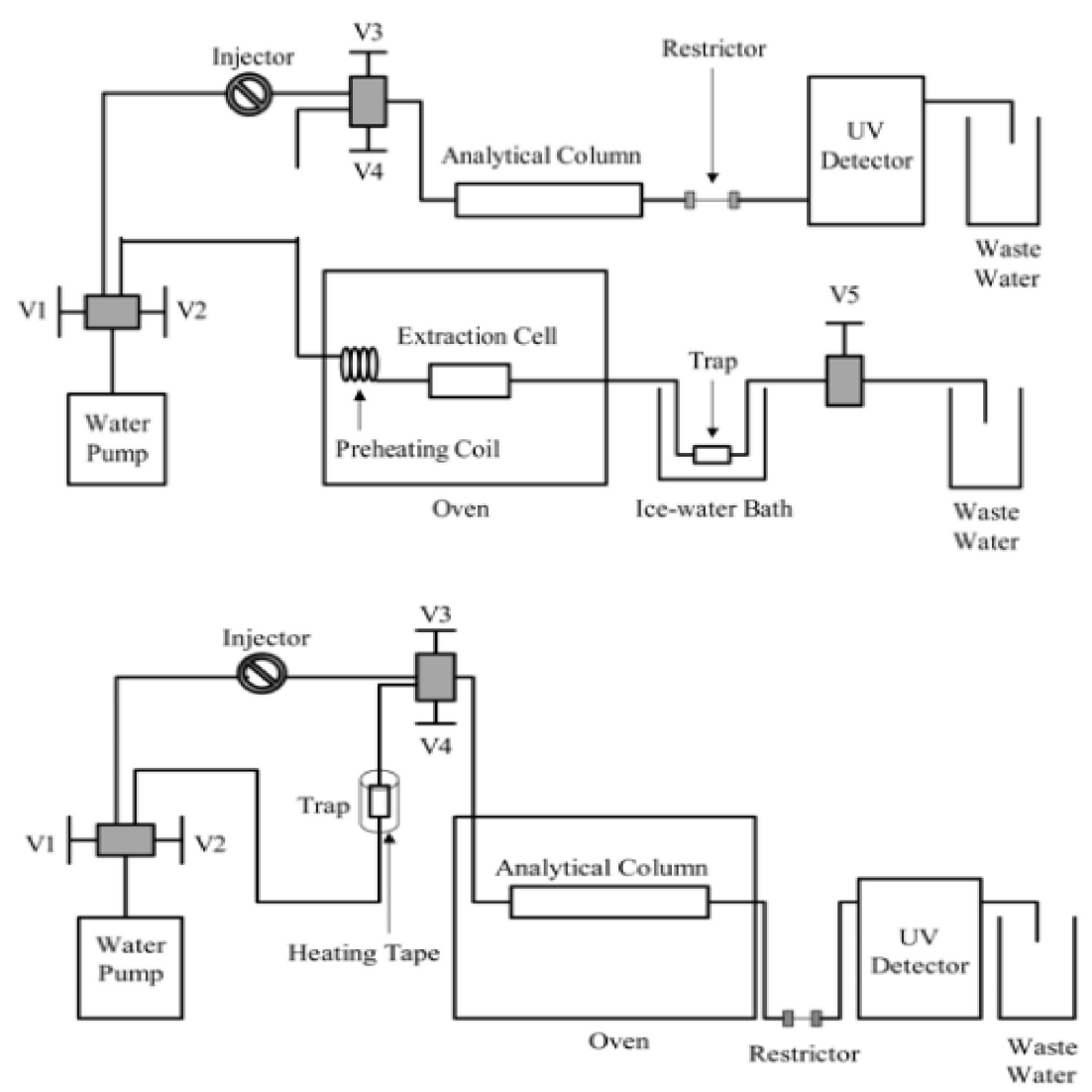

Figure 5. Offline coupling of SBWE with SBWC. Adapted with permission from [70] (Lamm, L.; Yang, Y. Off-line coupling of SBWE with subcritical water chromatography via a sorbent trap and thermal desorption. Anal. Chem. 2003, 75, 2237-2242.). Copyright 2003 American Chemical Society.

\section{Compounds Extracted by Subcritical Water}

\subsection{Flavonoids}

Flavonoids, also known as bioflavonoids, are widely found in plants and berries. They are important natural compounds in human diets. They have been used to prevent and treat cardiovascular diseases. In addition, they have strong antioxidant activities and antibacterial activities. When high-flavonoid apples were fed to healthy mice, decreases in some inflammation markers were reported [73].

Generally speaking, flavonoids belong to $\mathrm{pHenols.} \mathrm{Since} \mathrm{they} \mathrm{are} \mathrm{widely} \mathrm{investigated,}$ they can be presented separately from pHenols. Flavonoids have the general structure of a 15-carbon skeleton by connecting two benzene rings with a heterocyclic ring. The basic nucleus is 2-phenylchromone. Flavonoid compounds are usually poorly soluble in ambient water and most organic solvents. Table 1 summarizes SBWE of flavonoids from plant materials. 
Table 1. SBWE of flavonoids.

\begin{tabular}{|c|c|c|c|c|c|c|c|}
\hline Samples & $\begin{array}{l}\text { Medicinal } \\
\text { Parts }\end{array}$ & $\begin{array}{l}\text { Compounds } \\
\text { Extracted }\end{array}$ & $\begin{array}{l}\text { Extracts } \\
\text { Activity }\end{array}$ & $\begin{array}{l}\text { Extraction } \\
\text { Conditions }\end{array}$ & $\begin{array}{l}\text { Analytical } \\
\text { Methods }\end{array}$ & $\begin{array}{l}\text { Other Extraction } \\
\text { Methods (Solvent, } \\
\text { Ratios of Yields) }\end{array}$ & Ref. \\
\hline $\begin{array}{l}\text { Panax ginseng } \\
\text { C.A. Meyer }\end{array}$ & $\begin{array}{l}\text { stem } \\
\text { leave }\end{array}$ & $\begin{array}{l}\mathrm{TP} \text { and } \\
\text { flavonoids }\end{array}$ & antibacterial & $\begin{array}{l}110 \text { and } 165^{\circ} \mathrm{C}, \\
15 \mathrm{~min} \\
190^{\circ} \mathrm{C}, 10 \mathrm{~min}\end{array}$ & TEM, UV & $\begin{array}{l}\text { heating (water 95.4\%; } \\
\text { ethanol 91.3\%) }\end{array}$ & [15] \\
\hline $\begin{array}{l}\text { Chamomilla } \\
\text { matricaria L. }\end{array}$ & flowers & $\begin{array}{l}\mathrm{TP}, \mathrm{TF}, 18 \\
\text { polyphenolic } \\
\text { compounds, } \\
\text { apigenin }\end{array}$ & $\begin{array}{l}\text { antioxidant, } \\
\text { enzyme } \\
\text { inhibitory } \\
\text { activity }\end{array}$ & $\begin{array}{c}65-210^{\circ} \mathrm{C} \\
5-60 \mathrm{~min} \\
1: 30- \\
1: 100 \mathrm{~g} / \mathrm{mL}\end{array}$ & $\begin{array}{l}\text { TLC, UV, } \\
\text { HPLC-MS }\end{array}$ & & [18] \\
\hline Allium сера & onion wastes & $\begin{array}{l}\text { quercetin-4'- } \\
\text { glycoside, } \\
\text { quercetin, etc. }\end{array}$ & & $\begin{array}{c}40-160{ }^{\circ} \mathrm{C}, \\
5 \mathrm{~min}, 5 \mathrm{Mpa}, \\
1-10 \mathrm{~mm}, \\
\text { pH 3.0-7.0 }\end{array}$ & $\begin{array}{l}\text { LC-MS/MS } \\
\text { HPLC-UV }\end{array}$ & $\begin{array}{c}\text { convention (methanol } \\
\text { and hydrochloric acid } \\
94.3 \% \text { ) }\end{array}$ & [19] \\
\hline $\begin{array}{l}\text { Crocus sativus } \\
\text { L. }\end{array}$ & stigmas & $\begin{array}{l}\text { TP, dodecane, } \\
\gamma \text {-terpinene, } \\
\text { tetradecane, } \\
\text { etc. }\end{array}$ & $\begin{array}{c}\text { antioxidant } \\
\text { (DPPH, FRAP), } \\
\text { antibacterial }\end{array}$ & $\begin{array}{l}100-180^{\circ} \mathrm{C}, \\
10-30 \mathrm{~min}, \\
1: 10 \mathrm{~g} / \mathrm{mL}\end{array}$ & $\begin{array}{l}\text { GC/MS, } \\
\text { UV-vis }\end{array}$ & & [21] \\
\hline $\begin{array}{l}\text { Saururus } \\
\text { chinensis, etc. }\end{array}$ & $\begin{array}{l}\text { skin, leave, } \\
\text { peel, etc. }\end{array}$ & $\begin{array}{l}\text { quercetin, } \\
\text { isorhamnetin, } \\
\text { kaempferol, } \\
\text { isoquercitrin, } \\
\text { etc. }\end{array}$ & & $\begin{array}{c}10 \mathrm{Mpa}, \\
110-200{ }^{\circ} \mathrm{C}, \\
5-15 \mathrm{~min}\end{array}$ & HPLC & & [30] \\
\hline $\begin{array}{l}\text { Camellia } \\
\text { sinensis }\end{array}$ & leaves & $\begin{array}{l}\text { epigallocatechin } \\
\text { gallate }\end{array}$ & & $\begin{array}{c}80-120{ }^{\circ} \mathrm{C} \\
3-7 \mathrm{~min} \\
40-60 \mathrm{~mL} / \mathrm{g}\end{array}$ & HPLC & convention (water 87.6\%) & [44] \\
\hline $\begin{array}{l}\text { Origanum } \\
\text { vulgare L. }\end{array}$ & leaves & $\begin{array}{c}\text { TP, flavanone, } \\
\text { flavone, } \\
\text { flavanol }\end{array}$ & $\begin{array}{c}\text { antioxidant } \\
\text { (DPPH, TEAC, } \\
\text { ABTS) }\end{array}$ & $\begin{array}{c}10.34 \mathrm{Mpa}, 30 \\
\text { or } 15 \mathrm{~min} \\
25-200^{\circ} \mathrm{C} \\
\end{array}$ & $\begin{array}{l}\text { HPLC- } \\
\text { DAD, UV }\end{array}$ & & [47] \\
\hline orange & peels & $\begin{array}{l}\text { reducing sugar, } \\
\text { TP, pectin, } \\
\text { hesperidin, } \\
\text { narirutin }\end{array}$ & $\begin{array}{l}\text { antioxidant } \\
\text { (DPPH, FRAP) }\end{array}$ & $\begin{array}{c}110-150^{\circ} \mathrm{C} \\
10-30 \mathrm{~mL} / \mathrm{min} \\
10 \mathrm{Mpa}\end{array}$ & $\begin{array}{l}\text { HPLC, } \\
\text { UV-vis }\end{array}$ & $\begin{array}{l}\text { Soxhlet (ethanol 79.2\%), } \\
\text { shaker (ethanol 250\%), } \\
\text { UAE (ethanol 114\%) }\end{array}$ & [50] \\
\hline orange & peels & $\begin{array}{c}\text { flavones, } 7- \\
\text { hydroxyflavone }\end{array}$ & & $\begin{array}{l}100-150{ }^{\circ} \mathrm{C}, \\
0.5 \mathrm{~mL} / \mathrm{min}\end{array}$ & GC-FID & $\begin{array}{l}\text { UAE (methylene } \\
\text { chloride) }\end{array}$ & [70] \\
\hline $\begin{array}{l}\text { Citrus unshiu } \\
\text { Markovich }\end{array}$ & peels & $\begin{array}{l}\text { rutin, naringin, } \\
\text { hesperidin, } \\
\text { naringenin }\end{array}$ & & $\begin{array}{l}0.5-14 \mathrm{Mpa}, \\
5-15 \mathrm{~min}, \\
100-190^{\circ} \mathrm{C}\end{array}$ & HPLC & & [74] \\
\hline Allium cepa L. & peels & $\begin{array}{c}\mathrm{TP}, \mathrm{TF}, \\
\text { quercetin }\end{array}$ & $\begin{array}{c}\text { antioxidant } \\
\text { (DPPH, TBA, } \\
\text { FTC) }\end{array}$ & $\begin{array}{c}110 \text { and } 165^{\circ} \mathrm{C} \\
15 \mathrm{~min}, \mathrm{p}< \\
3.4 \mathrm{Mpa}\end{array}$ & HPLC, UV & $\begin{array}{l}\text { heating (ethanol } 153 \% \text {; } \\
\text { water } 45.6 \% \text { ) }\end{array}$ & [75] \\
\hline $\begin{array}{l}\text { Hippophae } \\
\text { rhamnoides }\end{array}$ & leaves & $\begin{array}{c}\mathrm{TP}, \mathrm{TF}, \\
\text { isorhamnetin, } \\
\text { kaempferol, } \\
\text { quercetin }\end{array}$ & $\begin{array}{l}\text { antioxidant, } \\
\text { cytotoxicity }\end{array}$ & $\begin{array}{l}25-200^{\circ} \mathrm{C}, \\
15 \mathrm{~min}, \\
10.34 \mathrm{Mpa}\end{array}$ & HPLC, UV, FM & $\begin{array}{c}\text { maceration (water } 21.3 \% \text { ), } \\
\text { Soxhlet (ethanol } 64.6 \% \text { ) }\end{array}$ & [76] \\
\hline Allium cepa L. & peels & $\begin{array}{c}\text { TP, TF, } \\
\text { kaempferol, } \\
\text { quercetin }\end{array}$ & $\begin{array}{l}\text { antioxidant } \\
\text { (DPPH) }\end{array}$ & $\begin{array}{c}170-230^{\circ} \mathrm{C} \\
3 \mathrm{Mpa}, 30 \mathrm{~min} \\
\text { pH } 2-10\end{array}$ & HPLC, UV-vis & heating (ethanol 26.7\%) & [77] \\
\hline $\begin{array}{c}\text { Achillea } \\
\text { millefolium } \mathrm{L} \text {. }\end{array}$ & herbal dust & $\begin{array}{l}\mathrm{TP}, \mathrm{TF}, \mathrm{HMF} \\
\text { chlorogenic } \\
\text { acid }\end{array}$ & $\begin{array}{c}\text { antioxidant } \\
\text { (DPPH, TEAC, } \\
\text { ABTS) }\end{array}$ & $\begin{array}{c}120-200{ }^{\circ} \mathrm{C}, \\
10-30 \mathrm{~min} \\
0-1.5 \% \mathrm{HCl} \\
3 \mathrm{Mpa}\end{array}$ & HPLC, UV-vis & & [78] \\
\hline $\begin{array}{l}\text { Curculigo } \\
\text { latifolia }\end{array}$ & root & $\begin{array}{c}\mathrm{TP}, \mathrm{TF}, \\
\text { pomiferin, etc. }\end{array}$ & $\begin{array}{c}\text { antioxidant } \\
\text { (DPPH, ABTS, } \\
\text { TEAC) }\end{array}$ & $\begin{array}{l}100-200{ }^{\circ} \mathrm{C} \\
10 \mathrm{Mpa} \\
30-120 \mathrm{~min}, \\
0.5 \mathrm{~mL} / \mathrm{min}\end{array}$ & LC-MS, UV & & [79] \\
\hline
\end{tabular}


Table 1. Cont.

\begin{tabular}{|c|c|c|c|c|c|c|c|}
\hline Samples & $\begin{array}{l}\text { Medicinal } \\
\text { Parts }\end{array}$ & $\begin{array}{l}\text { Compounds } \\
\text { Extracted }\end{array}$ & $\begin{array}{l}\text { Extracts } \\
\text { Activity }\end{array}$ & $\begin{array}{l}\text { Extraction } \\
\text { Conditions }\end{array}$ & $\begin{array}{l}\text { Analytical } \\
\text { Methods }\end{array}$ & $\begin{array}{l}\text { Other Extraction } \\
\text { Methods (Solvent, } \\
\text { Ratios of Yields) }\end{array}$ & Ref. \\
\hline Citrus unshiu & peels & $\begin{array}{l}\text { hesperidin and } \\
\text { narirutin }\end{array}$ & & $\begin{array}{c}110-190^{\circ} \mathrm{C} \\
3-15 \mathrm{~min}\end{array}$ & HPLC & & [80] \\
\hline Glycine max & okara & $\begin{array}{l}\text { genistin, } \\
\text { daidzin, } \\
\text { genistein, } \\
\text { daidzein }\end{array}$ & & $\begin{array}{c}100-200{ }^{\circ} \mathrm{C} \\
5 \mathrm{~min}, \\
2-5 \mathrm{Mpa}, \\
10-30 \mathrm{~g} / \mathrm{mL}\end{array}$ & HPLC & Soxhlet (methanol, 108\%) & [81] \\
\hline onion & skins & $\begin{array}{l}\text { quercetin, } \\
\text { quercetin- } 4^{\prime}- \\
\text { glucoside }\end{array}$ & & $\begin{array}{l}100-190{ }^{\circ} \mathrm{C} \\
\text { 5-30 min, } \\
9-13 \mathrm{Mpa}\end{array}$ & HPLC & $\begin{array}{c}\text { convention (methanol, } \\
92.8 \%)\end{array}$ & [82] \\
\hline Puerariae lobata & root & $\begin{array}{c}\text { puerarin, } \\
\text { daidzin, } \\
\text { daidzein } \\
\text { 3- } \\
\text { methoxypuerarin }\end{array}$ & & $\begin{array}{c}100-200{ }^{\circ} \mathrm{C} \\
15-75 \mathrm{~min} \\
1: 10-1: 25 \mathrm{~g} / \mathrm{mL}\end{array}$ & HPLC & $\begin{array}{l}\text { reflux (ethanol 91.6\%), } \\
\text { UAE (water 95.9\%) }\end{array}$ & [83] \\
\hline $\begin{array}{l}\text { Coriandrum } \\
\text { satioum }\end{array}$ & seeds & $\mathrm{TP}, \mathrm{TF}$ & $\begin{array}{l}\text { antioxidant } \\
\text { (DPPH) }\end{array}$ & $\begin{array}{l}100-200{ }^{\circ} \mathrm{C} \\
10-30 \mathrm{~min} \\
\text { 3-9 Mpa }\end{array}$ & UV & & [84] \\
\hline Citrus unshiu & peels & $\begin{array}{l}\text { flavanones, } \\
\text { polymethoxy- } \\
\text { Flavones, } \\
\text { etc. }\end{array}$ & $\begin{array}{l}\text { anticancer, car- } \\
\text { dioprotectives }\end{array}$ & $\begin{array}{c}120-180{ }^{\circ} \mathrm{C}, \\
1.0- \\
2.0 \mathrm{~mL} / \mathrm{min} \\
5.0 \pm 0.1 \mathrm{Mpa}\end{array}$ & GC, HPLC & $\begin{array}{l}\text { convention (methanol } \\
75.0 \% \text {; ethanol } 41.6 \% \text {; } \\
\text { acetone } 17.2 \% \text { ) }\end{array}$ & [85] \\
\hline $\begin{array}{l}\text { Phlomis } \\
\text { umbrosa }\end{array}$ & whole part & $\begin{array}{l}\text { TP, TF, iridoids } \\
\text { glycosides }\end{array}$ & $\begin{array}{l}\text { antioxidant } \\
\text { (DPPH, ABTS) }\end{array}$ & $\begin{array}{l}110-200{ }^{\circ} \mathrm{C} \\
10 \mathrm{Mpa} \\
1-25 \mathrm{~min}\end{array}$ & $\begin{array}{l}\text { HPLC, } \\
\text { ESI-MS }\end{array}$ & $\begin{array}{l}\text { convention (ethanol; } \\
\text { methanol; water) }\end{array}$ & [86] \\
\hline $\begin{array}{l}\text { Actinidia } \\
\text { deliciosa }\end{array}$ & peels & $\mathrm{TP}, \mathrm{TF}$ & $\begin{array}{l}\text { antioxidant } \\
\text { (DPPH, ABTS, } \\
\text { FRAP) }\end{array}$ & $\begin{array}{c}120-160{ }^{\circ} \mathrm{C}, \\
0-30 \mathrm{~min}, \\
3 \mathrm{Mpa}, \\
\text { pH 2-5.5 }\end{array}$ & UV-vis, $\mathrm{pH}$ & $\begin{array}{c}\text { convention (ethanol } \\
81.9 \%)\end{array}$ & [87] \\
\hline $\begin{array}{l}\text { Scutellaria } \\
\text { baicalensis }\end{array}$ & root & $\begin{array}{c}\text { baicalin, } \\
\text { baicalein, } \\
\text { wogonin, } \\
\text { wogonoside }\end{array}$ & & $\begin{array}{l}110-160{ }^{\circ} \mathrm{C}, \\
10-90 \mathrm{~min}, \\
20-100 \mathrm{mesh}\end{array}$ & HPLC & HRE (ethanol 93.0\%) & [88] \\
\hline Citrus unshiu & pomaces & $\begin{array}{c}\text { TP, poly- } \\
\text { methoxylated } \\
\text { flavones, } \\
\text { sinensetin, etc. }\end{array}$ & $\begin{array}{l}\text { antioxidant } \\
(\mathrm{DPPH}, \mathrm{TP})\end{array}$ & $\begin{array}{l}25-250{ }^{\circ} \mathrm{C} \\
10-60 \mathrm{~min} \\
0.1-5.0 \mathrm{Mpa}\end{array}$ & HPLC, UV & & [89] \\
\hline citrus unshiu & peels & $\begin{array}{l}\text { hesperidin, } \\
\text { narirutin, } \\
\text { prunin, } \\
\text { naringenin, } \\
\text { sinensetin, etc. }\end{array}$ & $\begin{array}{l}\text { antioxidants } \\
\text { (DPPH, FRAP), } \\
\text { enzyme }\end{array}$ & $\begin{array}{l}145-175^{\circ} \mathrm{C}, \\
15 \mathrm{~min} \\
5 \mathrm{Mpa}, 0.75- \\
2.2 \mathrm{~mL} / \mathrm{min}\end{array}$ & HPLC & $\begin{array}{c}\text { 2M HCl extraction } 42.9 \% \\
2 \mathrm{M} \mathrm{NaOH} \text { extraction } \\
38.9 \%\end{array}$ & [90] \\
\hline citrus unshiu & peels & $\begin{array}{l}\text { hesperidin and } \\
\text { narirutin }\end{array}$ & & $\begin{array}{l}110-200{ }^{\circ} \mathrm{C} \\
5-20 \mathrm{~min} \\
10 \pm 1 \mathrm{Mpa}\end{array}$ & HPLC, MS/MS & $\begin{array}{l}\text { convention (ethanol } \\
56.4 \% \text {; methanol } 35.8 \% \text {; } \\
\text { water } 6.2 \% \text { ) }\end{array}$ & [91] \\
\hline $\begin{array}{l}\text { palatiferum } \\
\text { Radlk. }\end{array}$ & leaves & $\begin{array}{l}\mathrm{TP}, \mathrm{TF} \text {, protein, } \\
\text { saponin, sugar, } \\
\text { apigenin, } \\
\text { kaempferol }\end{array}$ & $\begin{array}{c}\text { antioxidants } \\
\text { (DPPH, FRAP, } \\
\text { ABTS) }\end{array}$ & $\begin{array}{c}110-270{ }^{\circ} \mathrm{C} \\
15 \mathrm{~min}, 8 \mathrm{Mpa} \\
1: 70 \mathrm{~g} / \mathrm{mL}\end{array}$ & HPLC, UV & $\begin{array}{l}\text { convention (water } 77.7 \% \text {; } \\
\text { methanol } 32.8 \% \text { ), Soxhlet } \\
\quad(\text { ethanol } 43.7 \%)\end{array}$ & [92] \\
\hline $\begin{array}{l}\text { Glycyrrhiza } \\
\text { uralensis Fisch. }\end{array}$ & root & $\begin{array}{l}\text { TP, TF, } \\
\text { liquiritin, } \\
\text { flavanone, } \\
\text { isoflavone }\end{array}$ & $\begin{array}{l}\text { antioxidants } \\
\text { (DPPH, ABTS) }\end{array}$ & $\begin{array}{c}80-320^{\circ} \mathrm{C}, \\
2-100 \mathrm{~min}, \\
7.0 \mathrm{Mpa}, \\
1: 30 \mathrm{~g} / \mathrm{mL}, \\
\mathrm{pH} 3-11\end{array}$ & $\begin{array}{c}\text { HPLC, } \\
\text { MS/MS, UPLC }\end{array}$ & $\begin{array}{c}\text { UAE (water 20.6\%; } \\
\text { ethanol } 44.9 \% \text { ), MAE } \\
\text { (water } 25.6 \% \text {; ethanol } \\
63.8 \% \text { ) }\end{array}$ & [93] \\
\hline Tagetes erecta L. & $\begin{array}{l}\text { flower } \\
\text { residues }\end{array}$ & $\begin{array}{l}\mathrm{TP}, \mathrm{TF}, 5-\mathrm{HMF}, \\
\text { reducing sugar, } \\
\text { free amino } \\
\text { acids }\end{array}$ & $\begin{array}{l}\text { antioxidants } \\
\text { (DPPH, ABTS) }\end{array}$ & $\begin{array}{c}80-260{ }^{\circ} \mathrm{C} \\
15-90 \mathrm{~min} \\
1: 20- \\
1: 60 \mathrm{~g} / \mathrm{mL}, 120 \mathrm{rpm}\end{array}$ & $\begin{array}{l}\text { HPLC-DAD, } \\
\text { UV }\end{array}$ & $\begin{array}{c}\text { leaching (water } 9.4 \% \text {; } \\
\text { methanol } 69.9 \% \text {; ethanol } \\
68.8 \% \text {; acetone } 94.0 \% \text { ), } \\
\text { UAE (water } 9.9 \% \text {; } \\
\text { methanol } 69.8 \% \text {; ethanol } \\
64.3 \% \text {; acetone } 87.6 \% \text { ) }\end{array}$ & [94] \\
\hline
\end{tabular}


Table 1. Cont.

\begin{tabular}{|c|c|c|c|c|c|c|c|}
\hline Samples & $\begin{array}{l}\text { Medicinal } \\
\text { Parts }\end{array}$ & $\begin{array}{l}\text { Compounds } \\
\text { Extracted }\end{array}$ & $\begin{array}{l}\text { Extracts } \\
\text { Activity }\end{array}$ & $\begin{array}{l}\text { Extraction } \\
\text { Conditions }\end{array}$ & $\begin{array}{l}\text { Analytical } \\
\text { Methods }\end{array}$ & $\begin{array}{l}\text { Other Extraction } \\
\text { Methods (Solvent, } \\
\text { Ratios of Yields) }\end{array}$ & Ref. \\
\hline Daucus carota & leaves & $\begin{array}{l}\text { polyphenols, } \\
\text { luteolin }\end{array}$ & & $\begin{array}{l}110-230{ }^{\circ} \mathrm{C}, \\
0-114 \mathrm{~min}, \\
4 \mathrm{Mpa}\end{array}$ & UV, PLC & & [95] \\
\hline $\begin{array}{c}\text { Matricaria } \\
\text { chamomilla L. }\end{array}$ & flowers & $\begin{array}{l}\mathrm{TP}, \mathrm{TF}, \\
\text { apigenin-7-O- } \\
\text { glucoside, } \\
\text { etc. }\end{array}$ & $\begin{array}{l}\text { antimicrobial, } \\
\text { cytotoxic } \\
\text { activity }\end{array}$ & $\begin{array}{c}200{ }^{\circ} \mathrm{C}, 40 \mathrm{~min}, \\
1: 50 \mathrm{~g} / \mathrm{mL}\end{array}$ & $\begin{array}{l}\text { UHPLC, HESI- } \\
\text { MS/MS, UV }\end{array}$ & $\begin{array}{l}\text { Soxhlet (ethanol 129\%), } \\
\text { MAE (ethanol 117\%), } \\
\text { UAE (ethanol 104\%) }\end{array}$ & [96] \\
\hline $\begin{array}{c}\text { Silybum } \\
\text { murianum L. }\end{array}$ & seeds & $\begin{array}{c}\text { taxifolin, } \\
\text { silychristin, } \\
\text { silydianin, and } \\
\text { silybin }\end{array}$ & & $\begin{array}{l}75-250^{\circ} \mathrm{C}, \\
40-60 \mathrm{~min}, \\
12.5 \mathrm{Mpa}, \\
0.1-0.5 \mathrm{~mm}\end{array}$ & HPLC & $\begin{array}{l}\text { convention (ethanol } \\
101 \% \text {; water } 43.6 \% \text { ) }\end{array}$ & [97] \\
\hline $\begin{array}{c}\text { Echinacea } \\
\text { purpurea L. }\end{array}$ & flowers & $\mathrm{TP}, \mathrm{TF}$ & $\begin{array}{c}\text { antioxidant } \\
\text { (TEAC, ABTS) }\end{array}$ & $\begin{array}{c}103.4- \\
216.56{ }^{\circ} \mathrm{C} \\
3 \mathrm{Mpa}, 5.86- \\
34.414 \mathrm{~min}\end{array}$ & UV-vis & & [98] \\
\hline $\begin{array}{l}\text { Humulus } \\
\text { lupulus }\end{array}$ & pellets & $\begin{array}{l}\text { TP, desmetylx- } \\
\text { anthohumol, } \\
\text { prenylflavonoids, } \\
\text { etc. }\end{array}$ & $\begin{array}{c}\text { anti- } \\
\text { inflammatory }\end{array}$ & $\begin{array}{c}50-200^{\circ} \mathrm{C} \\
30 \mathrm{~min}, 10 \mathrm{Mpa}\end{array}$ & $\begin{array}{l}\text { HPLC, } \\
\text { MS/MS }\end{array}$ & $\begin{array}{l}\text { convention (hexane } \\
17.2 \% \text {; ethanol } 105 \% \text { ) }\end{array}$ & [99] \\
\hline Kunzea ericoides & leaves & $\begin{array}{l}\text { TP, TF, 5-HMF, } \\
\text { quercetin, } \\
\text { catechin, } \\
\text { syringic acid, } \\
\text { etc. }\end{array}$ & $\begin{array}{c}\text { antioxidant } \\
\text { (DPPH, FRAP) }\end{array}$ & $\begin{array}{c}150-210{ }^{\circ} \mathrm{C}, \\
0-40 \mathrm{~min} \\
15-35 \mathrm{~g} / \mathrm{mL}, \\
4 \mathrm{Mpa}\end{array}$ & HPLC, UV & $\begin{array}{c}\text { convention (ethanol } \\
37.5 \%)\end{array}$ & [100] \\
\hline $\begin{array}{c}\text { Pistacia } \\
\text { atlantica subsp. } \\
\text { mutica }\end{array}$ & hull & $\begin{array}{l}\text { TP, kaffesaure, } \\
\text { ethyl vanillin, } \\
\text { flavanomarein, } \\
\text { etc. }\end{array}$ & $\begin{array}{l}\text { antioxidant } \\
(\mathrm{DPPH}), \\
\text { reducing } \\
\text { power }\end{array}$ & $\begin{array}{l}110-200{ }^{\circ} \mathrm{C}, \\
30-60 \mathrm{~min}, \\
10-50 \mathrm{~g} / \mathrm{mL}\end{array}$ & $\begin{array}{l}\text { HPLC-DAD, } \\
\text { UV }\end{array}$ & HWE $\left(85^{\circ} \mathrm{C} 42.8 \%\right)$ & [101] \\
\hline $\begin{array}{c}\text { Satureja } \\
\text { hortensis L. }\end{array}$ & whole part & $\begin{array}{c}\mathrm{TP}, \mathrm{TF}, \\
\text { rosmarinic } \\
\text { acid, rutin, } \\
\text { quercetin, etc. }\end{array}$ & $\begin{array}{c}\text { cytotoxic, } \\
\text { antibacterial }\end{array}$ & $\begin{array}{c}140{ }^{\circ} \mathrm{C}, 30 \mathrm{~min} \\
4 \mathrm{Mpa}, \\
1: 20 \mathrm{~g} / \mathrm{mL}\end{array}$ & $\begin{array}{l}\text { HPLC-PDA, } \\
\text { UV }\end{array}$ & $\begin{array}{c}\text { maceration (ethanol } \\
57.2 \% \text { ), Soxhlet (ethanol } \\
18.4 \% \text { ), UAE (ethanol } \\
69.2 \% \text { ), MAE (ethanol } \\
81.3 \% \text { ) }\end{array}$ & [102] \\
\hline Urtica dioica $\mathrm{L}$. & leaves & $\begin{array}{c}\mathrm{TP}, \mathrm{TF} \\
\text { twenty-seven } \\
\text { compounds }\end{array}$ & $\begin{array}{c}\text { cytotoxic, } \\
\text { antifungal, } \\
\text { antimicrobial }\end{array}$ & $\begin{array}{c}125^{\circ} \mathrm{C}, 30 \mathrm{~min}, \\
3.5 \mathrm{Mpa}, \\
1: 30 \mathrm{~g} / \mathrm{mL}\end{array}$ & $\begin{array}{l}\text { UHPLC-HESI- } \\
\text { MS/MS }\end{array}$ & $\begin{array}{l}\text { UAE (water } 48.5 \% \text { ), MAE } \\
\text { (water } 100 \% \text { ) }\end{array}$ & [103] \\
\hline $\begin{array}{l}\text { Chamomilla } \\
\text { recutita } \mathrm{R} .\end{array}$ & flowers & $\begin{array}{l}2 \text { flavonoids, } 4 \\
\text { esters, } 1 \text { amino } \\
\text { acid, } \\
11 \text { pHenols, } \\
\text { etc. }\end{array}$ & & $\begin{array}{c}150 \text { or } 200{ }^{\circ} \mathrm{C} \\
5.0 \pm 0.1 \mathrm{Mpa} \\
1.7 \mathrm{~mL} / \mathrm{min} \\
40 \mathrm{~min}\end{array}$ & $\begin{array}{l}\text { UV, HPLC, } \\
\text { GC-MS }\end{array}$ & & [104] \\
\hline Glycine max & okara & $\begin{array}{l}\text { TP, gallic acid, } \\
\text { syringic acid, } \\
\text { ferruric acid, } \\
\text { etc. }\end{array}$ & $\begin{array}{c}\text { antioxidant } \\
\text { (ABTS, DPPH, } \\
\text { FRAP) }\end{array}$ & $\begin{array}{c}150{ }^{\circ} \mathrm{C}, 4 \mathrm{Mpa}, \\
5-275 \mathrm{~min} \\
20 \mathrm{mg} / \mathrm{mL}\end{array}$ & UV, HPLC, & & [105] \\
\hline $\begin{array}{l}\text { Carménère } \\
\text { grape }\end{array}$ & pomace & $\begin{array}{c}\text { flavanols, } \\
\text { stilbenes, } \\
\text { and pHenolic } \\
\text { acids }\end{array}$ & & $\begin{array}{c}90-150{ }^{\circ} \mathrm{C}, \\
5 \mathrm{~min}, 10 \mathrm{Mpa}, \\
15-50 \% \\
\text { glycerol }\end{array}$ & UPLC-MS & & [106] \\
\hline $\begin{array}{l}\text { Zingiber } \\
\text { officinale }\end{array}$ & root & $\begin{array}{l}\mathrm{TP}, \mathrm{TF} \text {, four } \\
\text { macro- and } \\
\text { five } \\
\text { microelements }\end{array}$ & $\begin{array}{c}\text { antioxidant } \\
\text { (OH·, ABTS, } \\
\text { TRP, etc.) }\end{array}$ & $\begin{array}{c}80-180{ }^{\circ} \mathrm{C}, 1 \mathrm{~h}, \\
5 \mathrm{Mpa} \\
1: 10 \mathrm{~g} / \mathrm{mL}\end{array}$ & $\begin{array}{l}\text { UV-vis, } \\
\text { ICP-MS }\end{array}$ & convention (water, $62.5 \%$ ) & [107] \\
\hline $\begin{array}{l}\text { Momordica } \\
\text { foetida }\end{array}$ & leaves & $\begin{array}{l}\text { quercetin, } \\
\text { kaempferol, } \\
\text { isorhamnetin }\end{array}$ & & $\begin{array}{c}100-300{ }^{\circ} \mathrm{C} \\
5 \mathrm{~mL} / \mathrm{s} \\
6.9 \pm 1.4 \mathrm{Mpa} \\
\mathrm{psi}\end{array}$ & $\begin{array}{l}\text { UHPLC-q- } \\
\text { TOF-MS }\end{array}$ & & [108] \\
\hline
\end{tabular}


Ko and coworkers have investigated the relationship between flavonoid structure and SBWE. They found that flavonoids with an $\mathrm{OH}$ side chain were optimally extracted at lower temperatures than $\mathrm{O}-\mathrm{CH}_{3}$ and $\mathrm{H}$ side chains. The optimal temperatures of the glycoside forms are lower than that of the less polar aglycones [30]. Turner et al. found that different glycosidic compounds may be converted by their respective aglycones in less than 10 min reaction time in water from onion waste [20]. Similar results were obtained by Nkurunziza et al. [81] and Zhang et al. [83] who investigated the extraction of four flavonoids from okara and from Puerariae lobata, respectively.

Various works have been published on the extraction efficiency and extracting activities by co Mparing SBWE with hot water extraction (HWE) [15], maceration [76], Soxhlet [50,81], ultrasonic assisted extraction (UAE) [93,103], microwave assisted extraction (MAE) [93,103], and reflux extraction [83]. All of them found that SBWE was more effective or at least equivalent to other extraction methods if only using one solvent. Đurović and coworkers [103] have selected water as an appropriate solvent for further extraction based on the extraction efficiency of six various solvents (water, acetone, 96\% ethanol, methylene chloride, $n$-hexane, and ethyl acetate). SBWE has the highest total polyphenolics (TP) and flavonoids contents, and antioxidant activity. Cytotoxic activity and antimicrobial activities against Staphylococcus aureus were confirmed. Moreover, Marín et al. [99] has co Mpared a possible better fit of pressurized liquid extraction for prenylflavonoids and three solvents (hexane, ethanol, and water) were used. They concluded that pressurized liquid extraction using ethanol as a solvent after using hexane is better than only using water for prenylflavonoids. When a sequential extraction was used, where solvents were used in an order of increasing polarity (first hexane, later ethanol, and finally water), yields did not improve. Different extraction methods have notable influences on the selectivity of these processes.

As shown in Table 1, the effect of extraction temperature and time have greater i Mpacts on extraction efficiency than pressure and sample-extraction liquid ratio. The optimal extraction temperature and time are summarized in Figure 6. The optimal extraction temperature of flavonoids is between 150 and $200{ }^{\circ} \mathrm{C}$ and the range of extraction time is between 10 and $50 \mathrm{~min}$.

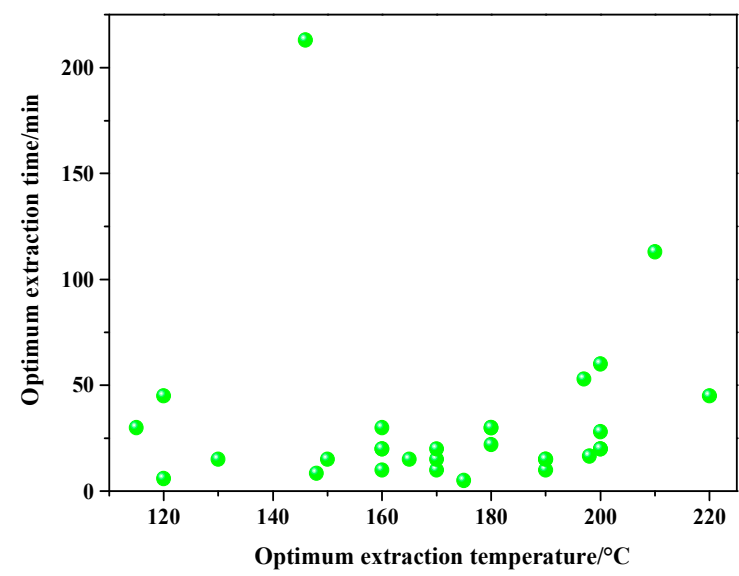

Figure 6. Optimum extraction conditions flavonoids.

\subsection{Polyphenols}

Polyphenols, also known as polyhydroxyphenols, are a structural class that is mainly natural, by the presence of more than one pHenolic unit and being deprived of nitrogenbased functions. Many fruits, vegetables, herbs, tea leaves, nuts, and algae contain high levels of naturally occurring pHenols. It has been reported that polyphenols can resist oxidation [31]. As shown in Table 2, extractions of polyphenols can be carried out either using a sole solvent such as water, methanol, ethanol or a mixture of solvents such as ethanol-water and methanol-water-formic acid. 
Table 2. Subcritical water extraction of polyphenols.

\begin{tabular}{|c|c|c|c|c|c|c|c|}
\hline Samples & $\begin{array}{l}\text { Medicinal } \\
\text { Parts }\end{array}$ & $\begin{array}{l}\text { Compounds } \\
\text { Extracted }\end{array}$ & $\begin{array}{l}\text { Extracts } \\
\text { Activity }\end{array}$ & $\begin{array}{l}\text { Extraction } \\
\text { Conditions }\end{array}$ & $\begin{array}{l}\text { Analytical } \\
\text { Methods }\end{array}$ & $\begin{array}{l}\text { Other Extraction } \\
\text { Methods (Solvent, } \\
\text { Ratios of Yields) }\end{array}$ & Ref. \\
\hline Allium ursinum L. & leaves & $\begin{array}{c}\text { TP, TF, 5-HMF, } \\
\text { catechin, } \\
\text { p-cumaric, } \\
\text { ferulic acids, etc. }\end{array}$ & $\begin{array}{c}\text { antioxidant } \\
\text { (DPPH, ABTS), } \\
\text { Millard } \\
\text { products }\end{array}$ & $\begin{array}{c}120-200{ }^{\circ} \mathrm{C}, \\
10-30 \mathrm{~min}, \\
0-1.5 \% \mathrm{HCl}, \\
1: 10 \mathrm{~g} / \mathrm{mL}\end{array}$ & HPLC-DAD & & [31] \\
\hline Terminalia chebula & fruits & $\begin{array}{l}\mathrm{TP}, \text { allic acid, } \\
\text { corilagin ellagic } \\
\text { acid }\end{array}$ & $\begin{array}{l}\text { antioxidant } \\
\text { (ABTS) }\end{array}$ & $\begin{array}{c}120-220^{\circ} \mathrm{C}, \\
2-4 \mathrm{~mL} / \mathrm{min} \\
4 \mathrm{Mpa}\end{array}$ & $\begin{array}{l}\text { TLC, UV, MS, } \\
\text { NMR, HPLC }\end{array}$ & $\begin{array}{l}\text { Soxhlet (water } 74.5 \% \text {; } \\
\text { ethanol } 46.3 \% \text { ), HWE } \\
\text { (water } 46.3 \% \text { ) }\end{array}$ & {$[34]$} \\
\hline $\begin{array}{l}\text { Lycium } \\
\text { ruthenicum Murr. }\end{array}$ & fruits & $\begin{array}{c}\text { total } \\
\text { anthocyanin, } \\
\text { seven } \\
\text { anthocyanins }\end{array}$ & $\begin{array}{c}\text { antioxidant } \\
\text { (ABTS, DPPH) }\end{array}$ & $\begin{array}{l}110-170{ }^{\circ} \mathrm{C} \\
30-90 \mathrm{~min} \\
1-3 \mathrm{~min} / \mathrm{L}\end{array}$ & $\begin{array}{c}\text { HPLC, } \\
\text { UPLC-MS }\end{array}$ & $\begin{array}{c}\text { UAE (water 59.8\%; } \\
\text { methanol } 81.1 \% \text { ) }\end{array}$ & [35] \\
\hline $\begin{array}{c}\text { Punica granatum } \\
\text { L. }\end{array}$ & peels & $\begin{array}{l}\mathrm{TP}, \mathrm{TF} \text {, punicalin, } \\
\text { etc. }\end{array}$ & & $\begin{array}{l}100-220{ }^{\circ} \mathrm{C} \\
5-30 \mathrm{~min} \\
3.0 \mathrm{Mpa}\end{array}$ & UV-vis, HPLC & $\begin{array}{l}\text { MAE (water } 121 \% \text {; } \\
\text { ethanol } 146 \% \text { ) }\end{array}$ & [109] \\
\hline Castanea sativa & shells & $\begin{array}{c}\text { tannins, pHeno- } \\
\text { lic acids, } \\
\text { flavonoids, } \\
\text { anthocyanins }\end{array}$ & $\begin{array}{c}\text { antioxidant } \\
\text { (DPPH, FRAP, } \\
\text { ABTS) }\end{array}$ & $\begin{array}{l}51-249{ }^{\circ} \mathrm{C} \\
6-30 \mathrm{~min}\end{array}$ & $\begin{array}{l}\text { UV-vis, } \\
\text { LC/ESI-MS }\end{array}$ & & [110] \\
\hline Salvia officinalis L. & by-products & $\mathrm{TP}, \mathrm{TF}$ & $\begin{array}{l}\text { antioxidant } \\
\text { (DPPH, TEAC, } \\
\text { reducing } \\
\text { power) }\end{array}$ & $\begin{array}{c}120-220{ }^{\circ} \mathrm{C}, \\
10-30 \mathrm{~min}, \\
3 \mathrm{Mpa}, 0-1.5 \% \\
\mathrm{HCl}\end{array}$ & UV & $\begin{array}{c}\text { maceration (water } \\
59.9 \% \text { ) }\end{array}$ & [111] \\
\hline Pistacia vera L. & hulls & $\begin{array}{l}\text { gallotannin, } \\
\text { anacardic acid, } \\
\text { etc. }\end{array}$ & $\begin{array}{c}\text { antioxidant } \\
\text { (ABTS, FRAP) }\end{array}$ & $\begin{array}{c}110-190^{\circ} \mathrm{C}, \\
6.9 \mathrm{Mpa}, \\
4 \mathrm{~mL} / \mathrm{min}\end{array}$ & 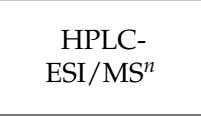 & $\begin{array}{l}\text { UAE (methanol } \\
83.9 \%) \text { ) }\end{array}$ & [112] \\
\hline Zingiber officinale & pulp and peel & $\begin{array}{l}\text { 6-gingerol, } \\
\text { 6-shogaol }\end{array}$ & $\begin{array}{l}\text { antioxidant } \\
\text { (FRAP) }\end{array}$ & $\begin{array}{c}10 \mathrm{Mpa}, \\
110-190{ }^{\circ} \mathrm{C}, \\
5-40 \mathrm{~min}\end{array}$ & HPLC & $\begin{array}{c}\text { convention } \\
\text { (methanol 114\%; } \\
\text { water } 77.1 \% \text { ) }\end{array}$ & [113] \\
\hline Sorfhum bicolor L. & bran & $\begin{array}{l}\mathrm{TP} \text {, oligomeric } \\
\text { procyanidins, } \\
\text { taxifolin, } \\
\text { taxifolin } \\
\text { hexoside }\end{array}$ & $\begin{array}{c}\text { antioxidant } \\
\text { (DPPH, ABTS), } \\
\text { antiprolifera- } \\
\text { tive }\end{array}$ & $\begin{array}{c}110-190{ }^{\circ} \mathrm{C}, \\
5-40 \mathrm{~min}, \\
1: 10-1: 50 \mathrm{~g} / \mathrm{mL}\end{array}$ & $\begin{array}{c}\text { HPLC, } \\
\text { ESI-MS/MS }\end{array}$ & heating (water $74.9 \%$ ) & [114] \\
\hline Nelumbo nucifera & seed epicarp & $\begin{array}{l}\text { TP, proantho- } \\
\text { cyanidin dimers, } \\
\text { trimer, cyanidin, } \\
\text { etc. }\end{array}$ & $\begin{array}{l}\text { antiproliferation } \\
\text { effect (MTT) }\end{array}$ & $\begin{array}{c}100-180{ }^{\circ} \mathrm{C}, \\
5-25 \mathrm{~min}, 1: 20- \\
1: 60 \mathrm{~g} / \mathrm{mL}, \\
1-5 \% \mathrm{NaHSO}_{3}\end{array}$ & $\begin{array}{c}\text { HPLC-ESI-MS, } \\
\text { UV }\end{array}$ & HWE (water 33.9\%) & [115] \\
\hline $\begin{array}{c}\text { German } \\
\text { chamomile }\end{array}$ & flowers & $\begin{array}{l}9 \text { pHenolic acids } \\
\text { and derivatives }\end{array}$ & $\begin{array}{l}\text { antioxidant, } \\
\text { cytotoxic, } \\
\text { enzyme }\end{array}$ & $\begin{array}{c}100{ }^{\circ} \mathrm{C} \\
1-9 \mathrm{Mpa}, \\
30 \mathrm{~min}\end{array}$ & $\begin{array}{l}\text { UHPLC-DAD, } \\
\text { MS/MS }\end{array}$ & & [116] \\
\hline $\begin{array}{l}\text { Fagopyrum } \\
\text { tataricum }\end{array}$ & grains & $\begin{array}{c}\text { pHenols, } \\
13 \text { pHenolics, } 4 \\
\text { flavonoids, } 3 \\
\text { anthocyanins }\end{array}$ & $\begin{array}{l}\text { antioxidant } \\
\text { (TEAC, CAA } \\
\text { and FRAP), } \\
\text { cytotoxicity }\end{array}$ & $\begin{array}{c}220{ }^{\circ} \mathrm{C}, 60 \mathrm{~min}, \\
5 \mathrm{Mpa}, \\
1: 60 \mathrm{~g} / \mathrm{mL}\end{array}$ & HPLC-MS, UV & UAE (water $83.5 \%$ ) & [117] \\
\hline A. uva-ursi & herbal dust & $\mathrm{TP}, \mathrm{TF}$ & $\begin{array}{l}\text { antioxidant } \\
\text { (DPPH, } \\
\text { reducing } \\
\text { power) }\end{array}$ & $\begin{array}{c}120- \\
220{ }^{\circ} \mathrm{C}, 3 \mathrm{Mpa}, \\
10-30 \mathrm{~min}, \\
0-1.5 \% \mathrm{HCl}\end{array}$ & UV & $\begin{array}{c}\text { maceration (water } \\
38.5 \% \text {; ethanol } 69.5 \% \text { ) }\end{array}$ & [118] \\
\hline $\begin{array}{l}\text { Hippophä̈ } \\
\text { rhamnoides L. }\end{array}$ & seed residue & $\begin{array}{l}\mathrm{TP}, \mathrm{TF}, \text { proantho- } \\
\text { cyanidins }\end{array}$ & $\begin{array}{l}\text { antioxidant } \\
\text { (DPPH) }\end{array}$ & $\begin{array}{c}80-180{ }^{\circ} \mathrm{C}, \\
15-90 \mathrm{~min}, \\
1: 10- \\
1: 50 \mathrm{~g} / \mathrm{mL}, \\
6 \mathrm{Mpa}\end{array}$ & UV & $\begin{array}{c}\text { convention (water } \\
19.6 \% \text {; methanol } \\
104 \% \text {; ethanol } 80.0 \% \text { ) }\end{array}$ & [119] \\
\hline grape (Croatina) & pomace & $\mathrm{TP}, \mathrm{TF}$ & $\begin{array}{l}\text { antioxidant } \\
(\mathrm{DPPH})\end{array}$ & $\begin{array}{c}100-140{ }^{\circ} \mathrm{C}, \\
8-15 \mathrm{Mpa}, \\
1-2 \mathrm{~mL} / \mathrm{min}\end{array}$ & UV & $\begin{array}{c}\text { convention (water } \\
5.3 \% \text {; ethanol } 7.87 \% \text { ) }\end{array}$ & [120] \\
\hline
\end{tabular}


Table 2. Cont.

\begin{tabular}{|c|c|c|c|c|c|c|c|}
\hline Samples & $\begin{array}{l}\text { Medicinal } \\
\text { Parts }\end{array}$ & $\begin{array}{l}\text { Compounds } \\
\text { Extracted }\end{array}$ & $\begin{array}{l}\text { Extracts } \\
\text { Activity }\end{array}$ & $\begin{array}{l}\text { Extraction } \\
\text { Conditions }\end{array}$ & $\begin{array}{l}\text { Analytical } \\
\text { Methods }\end{array}$ & $\begin{array}{l}\text { Other Extraction } \\
\text { Methods (Solvent, } \\
\text { Ratios of Yields) }\end{array}$ & Ref. \\
\hline $\begin{array}{c}\text { Matricaria } \\
\text { chamomilla L. }\end{array}$ & flowers & $\begin{array}{l}\text { polyphenolic } \\
\text { compounds, etc. }\end{array}$ & $\begin{array}{l}\text { antioxidant, } \\
\text { cytotoxic, } \\
\text { enzyme } \\
\text { inhibitory }\end{array}$ & $\begin{array}{l}65-210^{\circ} \mathrm{C}, \\
30 \mathrm{~min} \\
4.5 \mathrm{Mpa}\end{array}$ & $\begin{array}{l}\text { UHPLC-ESI- } \\
\text { MS/MS, } \\
\text { UV }\end{array}$ & & [121] \\
\hline Nelumbo nucifera & seedpods & $\begin{array}{l}\mathrm{TP}, \mathrm{TF} \text {, proantho- } \\
\text { cyanidin dimer, } \\
\text { isoquercetin, etc. }\end{array}$ & $\begin{array}{l}\text { antioxidant, an- } \\
\text { tiproliferative } \\
\text { (HepG2) }\end{array}$ & $\begin{array}{c}100-180{ }^{\circ} \mathrm{C}, \\
30-70 \mathrm{~mL} / \mathrm{g}, \\
5-25 \mathrm{~min}, \\
1-6 \% \mathrm{NaHSO}_{3}\end{array}$ & $\begin{array}{l}\text { UV-Vis, HPLC, } \\
\text { ESI-MS }^{n}\end{array}$ & HWE (water 91.4\%) & [122] \\
\hline Vitis vinifera L. & grape pomace & $\begin{array}{l}\text { catechins, } \\
\text { flavonols, } \\
\text { tannins, proan- } \\
\text { thocyanidins, } \\
\text { etc. }\end{array}$ & $\begin{array}{c}\text { antioxidant } \\
\text { (DPPH, ABTS) }\end{array}$ & $\begin{array}{c}40-120^{\circ} \mathrm{C}, \\
10 \mathrm{~min}, \\
10.34 \mathrm{Mpa}, \\
10-40 \% \\
\text { NADES }\end{array}$ & $\begin{array}{c}\text { UV, } \\
\text { HPLC-ESI-MS }\end{array}$ & & [123] \\
\hline sweet chestnut & bark & $\begin{array}{l}\text { TP, tannins, } \\
\text { ellagic and gallic } \\
\text { acids, } \\
\text { ellagitannins, etc. }\end{array}$ & $\begin{array}{l}\text { antioxidant } \\
\text { (DPPH) }\end{array}$ & $\begin{array}{c}150-250{ }^{\circ} \mathrm{C}, \\
10-60 \mathrm{~min}, \\
10-30 \mathrm{~mL} / \mathrm{g} \\
4.5 \mathrm{Mpa}\end{array}$ & UV-Vis, HPLC & & [124] \\
\hline $\begin{array}{l}\text { Symphytum } \\
\text { officinale }\end{array}$ & root & $\mathrm{TP}, \mathrm{TF}$ & $\begin{array}{l}\text { antioxidant } \\
\text { (DPPH), } \\
\text { enzyme } \\
\text { inhibitory }\end{array}$ & $\begin{array}{c}120-200{ }^{\circ} \mathrm{C}, 10- \\
30 \mathrm{~min}, 0-1.5 \% \\
\mathrm{HCl}\end{array}$ & UV, ELISA & $\begin{array}{c}\text { UAE (methanol 2.5\%; } \\
\text { ethanol } 17.4 \% \text { ); } \\
\text { maceration } \\
\text { (methanol } 4.4 \% ; \\
\text { ethanol } 29.8 \% \text { ) }\end{array}$ & [125] \\
\hline Pinot Nero & grape skins & $\mathrm{TP}$ & & $\begin{array}{c}80-120{ }^{\circ} \mathrm{C} \\
2 \mathrm{~h}, 10 \mathrm{Mpa}, \\
2-5 \mathrm{~mL} / \mathrm{min}\end{array}$ & UV-Vis & & [126] \\
\hline Coffea arabica L. & $\begin{array}{l}\text { spent coffee } \\
\text { grounds }\end{array}$ & $\begin{array}{c}\text { TP, } \\
\text { caffeoylquinic } \\
\text { acid, } \\
\text { feruloylquinic } \\
\text { acid, etc. }\end{array}$ & $\begin{array}{c}\text { antioxidant } \\
\text { (DPPH, ABTS) }\end{array}$ & $\begin{array}{c}160-180{ }^{\circ} \mathrm{C}, \\
35-55 \mathrm{~min}, \\
14.1-26.3 \mathrm{~g} / \mathrm{L}\end{array}$ & $\begin{array}{l}\text { HPLC-ABTS }^{+}, \\
\text {MS, UV }\end{array}$ & & [127] \\
\hline Curcuma longa $\mathrm{L}$. & rhizomes & $\begin{array}{l}\text { curcumin, } \\
\text { demethoxycur- } \\
\text { cumin }\end{array}$ & & $\begin{array}{l}120-160{ }^{\circ} \mathrm{C}, \\
6-22 \mathrm{~min} \\
1-2.5 \mathrm{Mpa}\end{array}$ & $\begin{array}{l}\text { HPLC-UV, } \\
\text { SEM }\end{array}$ & & [128] \\
\hline Curcuma longa L. & rhizomes & $\begin{array}{c}\alpha \text {-phellandrene, } \\
\text { curcumin, } \\
\beta \text {-caryophyllene, } \\
\text { trans- } \beta \text { - } \\
\text { farnesene, } \\
\beta \text {-bisabolene, } \\
\gamma \text {-curcumin, etc. }\end{array}$ & & $\begin{array}{c}90-150{ }^{\circ} \mathrm{C} \\
1-4 \mathrm{~mL} / \mathrm{min} \\
2 \mathrm{Mpa} \\
0.5-1.5 \mathrm{~mm}\end{array}$ & $\begin{array}{l}\text { GC/GC-MS, } \\
\text { GC -FID }\end{array}$ & $\begin{array}{l}\text { HD }(80.7 \%), \text { Soxhlet } \\
\text { (n-hexane } 1.2 \text {-fold })\end{array}$ & [129] \\
\hline Curcuma longa L. & rhizomes & $\begin{array}{l}\text { curcumin, } \\
\text { demethoxycur- } \\
\text { cumin, } \\
\text { bisdemethoxy- } \\
\text { curcumin }\end{array}$ & & $\begin{array}{l}110-150{ }^{\circ} \mathrm{C}, \\
1-10 \mathrm{~min}, \\
0.5-10 \mathrm{Mpa}\end{array}$ & HPLC & $\begin{array}{c}\text { convention (ethanol, } \\
1.13 \text {-fold) }\end{array}$ & [130] \\
\hline Curcuma longa L. & rhizomes & $\begin{array}{l}\text { curcumin, } \\
\text { demethoxycur- } \\
\text { cumin, } \\
\text { bisdemethoxy- } \\
\text { curcumin }\end{array}$ & & $\begin{array}{c}90-250{ }^{\circ} \mathrm{C}, \\
\mathrm{pH} 1.0-5.5 \\
5.0 \mathrm{Mpa}, \\
0.5 \mathrm{~mL} / \mathrm{min}\end{array}$ & $\begin{array}{c}\text { HPLC, UPLC, } \\
\text { LC-MS }\end{array}$ & $\begin{array}{c}\text { Soxhlet (acetone, } \\
\text { 1.17-fold) }\end{array}$ & [131] \\
\hline
\end{tabular}

Most experiments have proven that SBWE is superior to maceration, Soxhlet, and MAE [109,112,125], whereas Vladić et al. concluded that the lower temperatures of SBWE are more convenient for the valorization of pomegranate peel and MAE is more efficient than SBWE for the extraction of pHenolics from pomegranate peel while obtaining a 5-hydroxymethylfurfural-free extract [109].

Procyanidins are a class of polyphenols found in fruits, vegetables, and grains with potent chemo preventive activities. Gao et al. [119] successfully extracted procyanidins with 
subcritical water from sorghum bran, lotus seed epicarp, lotus seed pod and sea buckthorn seed, respectively. The results showed that extracts by SBWE had better reducing power, and antioxidant ability and antiproliferative ability on human hepatoma G2 cells. Moreover, the antioxidant and antiproliferative activity were found to be positively correlated with polyphenol concentration. In addition, Gao et al. have found a similar result, as the antioxidant of the sea buckthorn seed residue extracts was highly correlated with the content of the polyphenols. They demonstrated that extraction time and the water to solid ratio were the major factors that affected the extraction yield of polyphenols and the 2,2'-azino-bis-3-ethylbenzthiazoline-6-sulphonic acid (ABTS) radical scavenging activity of the extracts, while temperature was expressed as a crucial factor.

Curcumin belongs to polyphenols and is unstable and poorly bioavailable. Kiamahalleh et al. [128] studied some parameters on SBWE extraction efficiency of curcumin. Optimum extraction conditions are a temperature of $140{ }^{\circ} \mathrm{C}$ and pressure of $10 \mathrm{Mpa}$. Other works on SBWE of curcumin can be found in literature [29,129-131].

Pinto and his co-workers [110] have evaluated the optimal SBWE conditions of antioxidants and polyphenols from chestnut shells using response surface methodology (RSM). The optimal extraction conditions were determined by RSM as $220^{\circ} \mathrm{C} / 30 \mathrm{~min}$. At $150^{\circ} \mathrm{C}$, TP and antioxidant activity was decreased, which may be caused by the degradation of the tannin and the formation of other reaction products. In addition, a concentration of under $0.1 \mu \mathrm{g} / \mathrm{mL}$ extracts was safe. The reuse of this chestnut shells by-product is beneficial to the profitability of agro-industry, and to the environmental and economic sustainability. The optimal extraction temperature and time for SBWE of polyphenols are depicted in Figure 7. One can see that the optimal extraction temperature is between 130 and $200{ }^{\circ} \mathrm{C}$, and the time is between 15 and $35 \mathrm{~min}$.

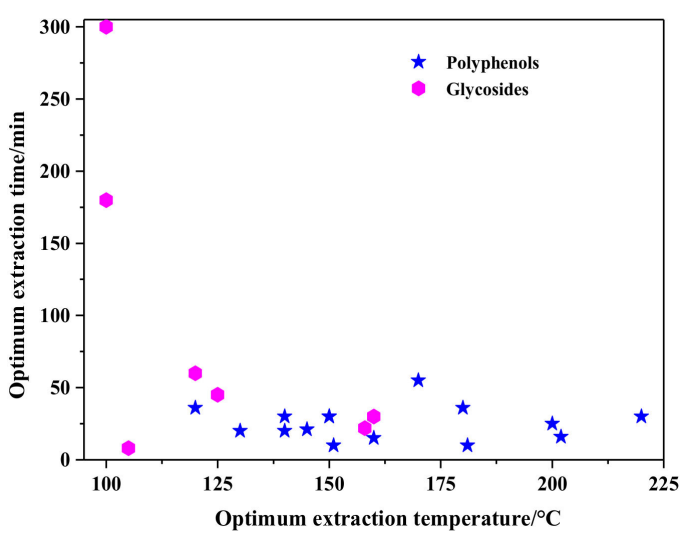

Figure 7. Optimum extraction conditions for polyphenols and glycosides.

\subsection{Organic Acids}

In general, organic acids in natural products are widely distributed in the leaves, roots, and fruits of the plants. The synthetic organic acids through chemical synthesis, enzymatic catalysis, and microbial fermentation are not discussed in this review. Organic acids are mostly soluble in water or ethanol and exhibit acidic properties, but they are difficult to dissolve in other organic solvents. It is generally believed that aliphatic organic acids have no special biological activity, but some natural organic acids such as citric acid, malic acid, tartaric acid, ascorbic acid, etc. have antibacterial, anti-inflammatory, hypoglycemic, antioxidant, and immune regulation effects. Depending on the organic acid in free state or in salt form, the extraction solvents could be water, dilute alkaline solution, diethyl ether, petroleum ether and cyclohexane, and other lipophilic organic solvents. A summary of recent studies on SBWE of organic acids are shown in Table 3. 
Table 3. Subcritical water extraction of organic acids.

\begin{tabular}{|c|c|c|c|c|c|c|c|}
\hline Samples & $\begin{array}{l}\text { Medicinal } \\
\text { Parts }\end{array}$ & $\begin{array}{l}\text { Compounds } \\
\text { Extracted }\end{array}$ & $\begin{array}{l}\text { Extracts } \\
\text { Activity }\end{array}$ & $\begin{array}{l}\text { Extraction } \\
\text { Conditions }\end{array}$ & $\begin{array}{l}\text { Analytical } \\
\text { Methods }\end{array}$ & $\begin{array}{l}\text { Other Extraction } \\
\text { Methods (Solvent, } \\
\text { Ratios of Yields) }\end{array}$ & Ref. \\
\hline $\begin{array}{c}\text { Panax ginseng } \\
\text { Meyer }\end{array}$ & root & $\begin{array}{l}\text { TP, chlorogenic } \\
\text { acid, caffeic acid, } \\
\text { gallic acid, etc. }\end{array}$ & $\begin{array}{l}\text { antioxidant } \\
\text { (DPPH, ABTS, } \\
\text { FRAP, HRS) }\end{array}$ & $\begin{array}{c}100-240{ }^{\circ} \mathrm{C} \\
15 \mathrm{~min}, \\
4-9 \mathrm{Mpa} \\
200 \mathrm{rpm}\end{array}$ & HPLC, UV & & [23] \\
\hline $\begin{array}{c}\text { Helicteres isora } \\
\text { L. }\end{array}$ & & $\begin{array}{c}\text { hexadecanoic } \\
\text { acid, octadecnoic } \\
\text { acid, heptadecen- } \\
\text { 8-carbonic acid } \\
\text { etc. }\end{array}$ & $\begin{array}{l}\text { antibiofilm, } \\
\text { antioxidant, } \\
\text { antimicrobial, } \\
\text { antienzymatic }\end{array}$ & $\begin{array}{c}160^{\circ} \mathrm{C}, 30 \mathrm{~min}, \\
1 \mathrm{Mpa}, 1: \\
30 \mathrm{~g} / \mathrm{mL}\end{array}$ & GC-MS, UV & & [27] \\
\hline $\begin{array}{l}\text { XiLan olive } \\
\text { fruit }\end{array}$ & olive dreg & $\begin{array}{l}\mathrm{TP} \text {, chlorogenic } \\
\text { acid, gallic acid, } \\
\text { syringic acid, etc. }\end{array}$ & $\begin{array}{l}\text { antioxidant } \\
\text { (ABTS, DPPH, } \\
\text { reducing } \\
\text { power) }\end{array}$ & $\begin{array}{c}100-180{ }^{\circ} \mathrm{C}, \\
5-60 \mathrm{~min}, \\
1: 20-1: 60 \mathrm{~g} / \mathrm{mL}\end{array}$ & $\begin{array}{c}\text { LC-MS-IT-TOF, } \\
\text { UV }\end{array}$ & $\begin{array}{c}\text { convention } \\
\text { (methanol 3.2\%; } \\
\text { ethanol } 0.6 \% \text {; DMK } \\
0.9 \% \text { ) }\end{array}$ & {$[33]$} \\
\hline $\begin{array}{c}\text { Camellia oleifera } \\
\text { Abel. }\end{array}$ & seeds & $\begin{array}{l}\text { free fatty acids } \\
\text { (palmitic acid, } \\
\text { stearate, oleic } \\
\text { acid, etc.), tea } \\
\text { saponin }\end{array}$ & $\begin{array}{l}\text { antioxidant } \\
\text { (DPPH) }\end{array}$ & $\begin{array}{c}60-160{ }^{\circ} \mathrm{C}, \\
2-7 \mathrm{Mpa}, \\
5-60 \mathrm{~min}, \\
1: 3-1: 25 \mathrm{~g} / \mathrm{mL}\end{array}$ & GC-MS, FT-IR & $\begin{array}{l}\text { Soxhlet (petroleum } \\
\text { ether } 100 \%), \text { cold } \\
\text { pressed }(100 \%)\end{array}$ & [37] \\
\hline $\begin{array}{c}\text { sunflower } \\
\text { seeds (Natura) }\end{array}$ & dehulled seeds & $\begin{array}{l}\text { total proteins, } \\
\text { total } \\
\text { carbohydrates, } \\
\text { TP }\end{array}$ & $\begin{array}{l}\text { antioxidant } \\
\text { capacities }\end{array}$ & $\begin{array}{c}\text { 60-160 }{ }^{\circ} \mathrm{C}, \\
5-120 \mathrm{~min}, \\
3 \mathrm{Mpa}, \\
1: 10-1: 30 \mathrm{~g} / \mathrm{mL}\end{array}$ & $\begin{array}{c}\text { GC-FID, } \\
\text { UV-Vis, HPLC }\end{array}$ & $\begin{array}{c}\text { Soxhlet (hexane } \\
67.3 \%)\end{array}$ & [38] \\
\hline $\begin{array}{l}\text { cottonseed } \\
\text { (Egypt) }\end{array}$ & cottonseed & $\begin{array}{l}\text { linoleic acid, } \\
\text { palmatic acid, } \\
\text { oleic acid, } \\
\text { myristic acid }\end{array}$ & & $\begin{array}{c}180-280{ }^{\circ} \mathrm{C} \\
5-60 \mathrm{~min} \\
1: 2-2: 1 \mathrm{~g} / \mathrm{mL}\end{array}$ & GC-FID & $\begin{array}{l}\text { heating (hexane } \\
89.5 \%)\end{array}$ & [39] \\
\hline $\begin{array}{l}\text { green coffee } \\
\text { (Robusta } \\
\text { Uganda) }\end{array}$ & beans & chlorogenic acid & & $\begin{array}{c}130-170{ }^{\circ} \mathrm{C}, \\
40-90 \mathrm{~min}, \\
0-30 \% \text { ethanol }\end{array}$ & HPLC & $\begin{array}{c}\text { convention (ethanol } \\
66.7 \% \text { ) }\end{array}$ & [45] \\
\hline $\begin{array}{l}\text { Nannochloropsis } \\
\text { gaditana }\end{array}$ & & $\begin{array}{c}\text { fatty acids, } \\
\text { omega-3, } \\
\text { omega-6, lipid }\end{array}$ & & $\begin{array}{c}156.1-273.9^{\circ} \mathrm{C}, \\
6.6-23.4 \mathrm{~min}, \\
33-117 \mathrm{~g} / \mathrm{L}\end{array}$ & GC-FID, SEM & $\begin{array}{c}\text { Soxhlet (n-hexane } \\
100 \%)\end{array}$ & [51] \\
\hline $\begin{array}{l}\text { Saccharina } \\
\text { japonica }\end{array}$ & & $\begin{array}{l}\text { gallic, caffeic, } \\
\text { vanillic, syringic, } \\
\text { chlorogenic, p- } \\
\text { hydroxybenzoic } \\
\text { acids, etc. }\end{array}$ & $\begin{array}{c}\text { antioxidant } \\
\text { (DPPH, ABTS, } \\
\text { total } \\
\text { antioxidant } \\
\text { (FRAP) }\end{array}$ & $\begin{array}{c}100-250^{\circ} \mathrm{C} \\
5 \mathrm{~min}, 5 \mathrm{Mpa}, \\
0.25-1.00 \mathrm{M} \mathrm{ILs}\end{array}$ & HPLC, UV & $\begin{array}{c}\text { convention (DMK } \\
0.2 \% ; \text { DCM 0.3\%; } \\
\text { Et }_{2} \mathrm{O} 0.8 \% ; \text { IL } 1.6 \% \text { ) }\end{array}$ & [52] \\
\hline $\begin{array}{c}\text { Haematococcus } \\
\text { pluvialis }\end{array}$ & & $\begin{array}{l}\text { p- } \\
\text { hydroxybenzoic } \\
\text { acid, gallic acid, } \\
\text { siringic acid, } \\
\text { vanillic acid, etc. }\end{array}$ & $\begin{array}{c}\text { antioxidant } \\
\text { (ABTS, TEAC), } \\
\text { antimicrobial } \\
\text { activity }\end{array}$ & $\begin{array}{l}50-200^{\circ} \mathrm{C}, \\
20 \mathrm{~min}, \\
10 \mathrm{Mpa}\end{array}$ & $\begin{array}{l}\text { HPLC-DAD- } \\
\text { MS, SEM, } \\
\text { GC-MS }\end{array}$ & & [53] \\
\hline $\begin{array}{l}\text { Momordica } \\
\text { charantia }\end{array}$ & fruits & $\begin{array}{l}\mathrm{TP}, \text { gallic acid, } \\
\text { gentisic acid, } \\
\text { chlorogenic acid }\end{array}$ & $\begin{array}{l}\text { antioxidant } \\
\text { (ABTS) }\end{array}$ & $\begin{array}{c}130-200{ }^{\circ} \mathrm{C} \\
10 \mathrm{Mpa} \\
2-5 \mathrm{~mL} / \mathrm{min}\end{array}$ & HPLC, UV & $\begin{array}{c}\text { Soxhlet (methanol } \\
4.9 \% \text { ), UAE } \\
\text { (methanol } 4.0 \% \text { ) }\end{array}$ & [132] \\
\hline $\begin{array}{l}\text { Morus nigra L., } \\
\text { Teucrium } \\
\text { chamaedrys L., } \\
\text { Geranium } \\
\text { macrorrhizum } \\
\text { L., Symphytum } \\
\text { officinale L. }\end{array}$ & leaves, flowers & $\begin{array}{l}\mathrm{TP} \text {, chlorogenic } \\
\text { acid, gallic acid, } \\
\text { vanillic acid, etc. }\end{array}$ & $\begin{array}{l}\text { antioxidant, } \\
\text { antifungal, } \\
\text { antibacterial, } \\
\text { cytotoxic }\end{array}$ & $\begin{array}{c}60-200^{\circ} \mathrm{C}, \\
30 \mathrm{~min}, 1 \mathrm{Mpa}, \\
1: 40 \mathrm{~g} / \mathrm{mL}\end{array}$ & $\begin{array}{l}\text { HPLC- } \\
\text { DADUV }\end{array}$ & & [133] \\
\hline $\begin{array}{l}\text { Prunus avium } \\
\text { L., Prunus } \\
\text { cerasus L. }\end{array}$ & stems & $\begin{array}{l}3 \text { alcohols, } 10 \\
\text { organic acids, } \\
\text { etc. }\end{array}$ & $\begin{array}{l}\text { antioxidant, an- } \\
\text { tiproliferative }\end{array}$ & $\begin{array}{c}150{ }^{\circ} \mathrm{C}, 30 \mathrm{~min}, \\
2 \mathrm{Mpa}\end{array}$ & GC-MS, UV & & [134] \\
\hline Castanea sativa & nuts & $\begin{array}{l}\text { ellagic acid, feru } \\
\text { lic acid, gallic } \\
\text { acid, etc. }\end{array}$ & antioxidant & $\begin{array}{l}120-135^{\circ} \mathrm{C} \\
15-60 \mathrm{~min}\end{array}$ & HPLC & & [135] \\
\hline
\end{tabular}


Table 3. Cont.

\begin{tabular}{|c|c|c|c|c|c|c|c|}
\hline Samples & $\begin{array}{l}\text { Medicinal } \\
\text { Parts }\end{array}$ & $\begin{array}{l}\text { Compounds } \\
\text { Extracted }\end{array}$ & $\begin{array}{l}\text { Extracts } \\
\text { Activity }\end{array}$ & $\begin{array}{l}\text { Extraction } \\
\text { Conditions }\end{array}$ & $\begin{array}{l}\text { Analytical } \\
\text { Methods }\end{array}$ & $\begin{array}{l}\text { Other Extraction } \\
\text { Methods (Solvent, } \\
\text { Ratios of Yields) }\end{array}$ & Ref. \\
\hline $\begin{array}{l}\text { Solanum } \\
\text { tuberosum }\end{array}$ & potato peel & $\begin{array}{l}\text { TP, gallic acid, } \\
\text { caffeic acid, } \\
\text { chlorogenic acid, } \\
\text { protocatechuic } \\
\text { acid, etc. }\end{array}$ & & $\begin{array}{l}100-240{ }^{\circ} \mathrm{C}, \\
30-120 \mathrm{~min}, \\
6 \mathrm{Mpa}\end{array}$ & HPLC, UV & $\begin{array}{l}\text { convention } \\
\text { (methanol 1.6\%; } \\
\text { ethanol } 2.0 \% \text { ) }\end{array}$ & [136] \\
\hline $\begin{array}{l}\text { Actinidia } \\
\text { deliciosa }\end{array}$ & pomace & $\begin{array}{l}\text { TP, chlorogenic } \\
\text { acid, } \\
\text { protocatechuic } \\
\text { acid, etc. }\end{array}$ & $\begin{array}{c}\text { antioxidant } \\
\text { (DPPH, FRAP, } \\
\text { ABTS) }\end{array}$ & $\begin{array}{l}170-225{ }^{\circ} \mathrm{C} \\
10-180 \mathrm{~min} \\
5 \mathrm{Mpa}\end{array}$ & UV, HPLC, pH & & [137] \\
\hline $\begin{array}{c}\text { hypnea } \\
\text { musciformis }\end{array}$ & & $\begin{array}{c}\text { chlorogenic, } \\
\text { protocatechuic, } \\
\text { and gallic acids, } \\
\mathrm{TP}, \mathrm{TF} \text {, etc. }\end{array}$ & $\begin{array}{c}\text { antioxidant } \\
\text { (DPPH, ABTS), } \\
\text { emulsify }\end{array}$ & $\begin{array}{c}120-270{ }^{\circ} \mathrm{C} \\
10 \mathrm{~min} \\
1: 50- \\
1: 150 \mathrm{~g} / \mathrm{mL}\end{array}$ & $\mathrm{pH}, \mathrm{UV}, \mathrm{HPLC}$ & & [138] \\
\hline Carica papaya L. & $\begin{array}{l}\text { papaya } \\
\text { seeds }\end{array}$ & $\begin{array}{l}\mathrm{TP}, 18 \text { pHenolic } \\
\text { acids, } 20 \\
\text { flavonoids, } 1 \\
\text { stilbene, etc. }\end{array}$ & $\begin{array}{l}\text { antioxidant } \\
\text { (DPPH, } \\
\beta \text {-carotene } \\
\text { bleaching) }\end{array}$ & $\begin{array}{l}70-150^{\circ} \mathrm{C}, \\
10 \mathrm{Mpa}, \\
1-40 \mathrm{~min}, \\
4 \mathrm{~mL} / \mathrm{min}\end{array}$ & $\begin{array}{l}\text { LC-ESI- } \\
\text { MS/MS, } \\
\text { UV }\end{array}$ & Soxhlet (water $37.1 \%$ ) & [139] \\
\hline $\begin{array}{l}\text { Zingiber } \\
\text { officinale }\end{array}$ & $\begin{array}{l}\text { ginger } \\
\text { rhizome }\end{array}$ & $\begin{array}{l}12 \text { sugars, } 8 \text { diols, } \\
4 \text { pHenolic acids, } \\
\text { etc. }\end{array}$ & $\begin{array}{c}\text { antimicrobial, } \\
\text { cytotoxic }\end{array}$ & $\begin{array}{l}150{ }^{\circ} \mathrm{C}, 1 \mathrm{~h}, \\
1: 10 \mathrm{~g} / \mathrm{mL}\end{array}$ & $\begin{array}{l}\text { HPLC-ESI- } \\
\text { TOFMS }\end{array}$ & heating (water) & [140] \\
\hline $\begin{array}{l}\text { Chlorella sp. } \\
\text { microalgae }\end{array}$ & & $\begin{array}{l}\text { TP, caffeic acid, } \\
\text { ferulic acid, } \\
\text { p-coumaric acid }\end{array}$ & $\begin{array}{l}\text { antioxidant } \\
\text { (DPPH) }\end{array}$ & $\begin{array}{l}100-250{ }^{\circ} \mathrm{C} \\
5-20 \mathrm{~min}\end{array}$ & $\begin{array}{l}\text { UV, SEM, } \\
\text { HPLC }\end{array}$ & & [141] \\
\hline Vitis vinifera & vine-canes & $\begin{array}{c}\mathrm{TP}, \\
\text { flavonoids, pHe- } \\
\text { nolic acids, } \\
\text { flavonols }\end{array}$ & $\begin{array}{l}\text { antioxidant, } \\
\text { antiradical }\end{array}$ & $\begin{array}{l}125-250^{\circ} \mathrm{C} \\
50 \mathrm{~min}\end{array}$ & HPLC, UV & & [142] \\
\hline $\begin{array}{l}\text { Cinnamomum } \\
\text { Cassia Blume }\end{array}$ & cinnamon & $\begin{array}{c}\text { coumarin, } \\
\text { cinnamic acid, } \\
\text { cinnamaldehyde, } \\
\text { cinnamyl alcohol, } \\
\text { etc. }\end{array}$ & & $\begin{array}{c}110-130{ }^{\circ} \mathrm{C}, \\
20-60 \mathrm{~min}, \\
2-4 \mathrm{Mpa} \\
1: 10 \mathrm{~g} / \mathrm{mL}\end{array}$ & HPLC & & {$[143]$} \\
\hline
\end{tabular}

The use of SBWE was explored for the extraction of gallic acid, chlorogenic acid, caffeic acid, ferulic acid, vanillic acid, and coumaric acid from various matrices. Inevitably, some other active components such as pHenolics [23], flavonoids [108], proteins [23], lipids, peptides, amino acids, and other organic compounds were often coextracted. Švarc-Gajić et al. [134] have used SBWE for the extraction of alcohols, organic acids, sugars, and other organic compounds from both sweet and sour cherry stems, finding the chemical compositions of the two samples similar. Harun et al. [51] reported lipid extraction with a relatively high content of eicosapentaenoic acid from Nannochloropsis gaditana, finding $237^{\circ} \mathrm{C}$ and $14 \mathrm{~min}$ to be the optimum extraction conditions.

Chun et al. [52] explored the power of the ionic liquid (IL)-assisted SBWE method in obtaining different pHenolic compounds from the brown seaweed Saccharina japonica and found the imidazolium-based IL 1-butyl-3-methylimidazolium tetrafluoroborate acted as a catalyst. Co Mpared with SBWE, SBWE + IL provided a progressive enhancement in extracting pHenolics. At $175^{\circ} \mathrm{C}$, the contents of gallic, chlorogenic, gentisic, protocatechuic, caffeic, and syringic acids in extracting products were approximately 1.18-, 4.68-, 4.66-, 7.67-, 5.12-, and 5.08-fold higher than in SBWE. However, $p$-hydroxybenzoic acid had a slight deficiency $\left(0.85\right.$-fold at $\left.175^{\circ} \mathrm{C}\right)$ and vanillic acid easily decomposed in SBWE + ILs.

Ravber et al. [38] have applied SBWE to simultaneous removal of oil extracts and water-soluble extracts. The optimal extraction yield of oil was obtained at $130{ }^{\circ} \mathrm{C}$ and mass/sample of 1:20 g/mL after $30 \mathrm{~min}$. Hydrolysis of ester and glycoside bonded antioxidants occurred, which produced oil with much higher antioxidant capacities than oil extracted using the Soxhlet ( $n$-hexane) method. In addition, Abdelmoez and coworkers [39] 
have also co Mpared the efficiency of water extracts and $n$-hexane extracts from cottonseed. The optimum oil extraction conditions are $270{ }^{\circ} \mathrm{C}, 30 \mathrm{~min}$, and the particle size is less than $0.5 \mathrm{~mm}$.

The optimal SBWE extraction temperature and time are shown in Figure 8. One can see that the optimal extraction temperature of organic acids is mainly between 130 and $240{ }^{\circ} \mathrm{C}$, and the time is between 10 and $50 \mathrm{~min}$.

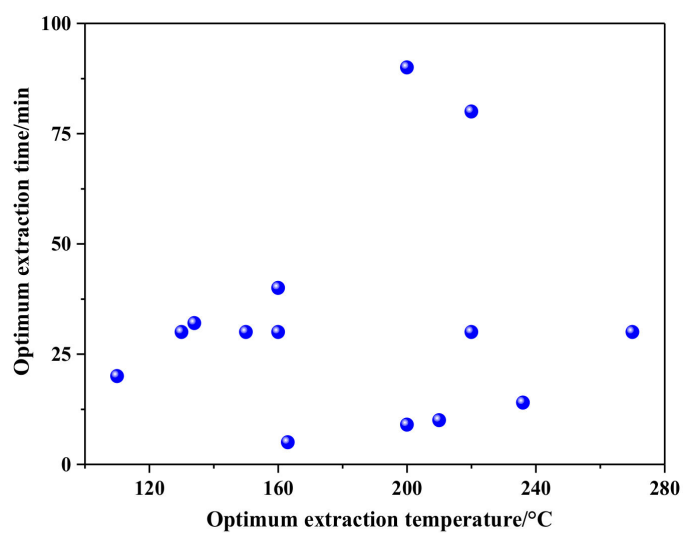

Figure 8. Optimum extraction conditions for organic acids.

\subsection{Glycosides}

Glycosides are compounds in which sugars or sugar derivatives are bound to another type of non-sugar substance (also called aglycones, ligands or substituents). Glycosides are linked by an O- N-, S-, or C-glycosidic bond between a sugar and a non-sugar component, which are widely found in the root, stems, leaves, flowers, and fruits of plants. Most glycosides are colored crystals, and generally a little bitter.

Glycosides extracted by SBWE have been proven to have antioxidant activities and tyrosinase inhibitory activity, as shown in Table 4 [36,144-152]. Gao et al. [144] has performed SBWE of pHenolic compounds from pomegranate seed residues at $80-280^{\circ} \mathrm{C}$. The results showed that TP increased with the rise of extraction temperature from $80^{\circ} \mathrm{C}$ to $220^{\circ} \mathrm{C}$ and decreased from $220^{\circ} \mathrm{C}$ to $280^{\circ} \mathrm{C}$. At $80-220^{\circ} \mathrm{C}$, the breakage of the bonds led to the increase of TP, however, a higher temperature caused the pHenolics to degrade. In addition, they co Mpared SBWE with leaching and UAE using water (room temperature) and organic solvents namely methanol, ethanol, and acetone. TP and antioxidant capacities of SBWE $\left(120^{\circ} \mathrm{C}\right)$ were not as high as organic solvents; however, with respect to the extraction time ( $2 \mathrm{~h}$ for leaching vs. $30 \mathrm{~min}$ for SBWE) and toxicity, subcritical water is more acceptable. Meng and Cheng [149] have studied 13 pHenolic compounds and 20 inorganic elements of Erigeron breviscapus. They also have found similar results, as the glycosides are not stable at a high temperature and with a long extraction time. For example, scutellarein and apigenine are the aglycones of corresponding acutellarin and apigenin 7-glucuronide, when at high temperature glycosidic bonds become unstable and begin to decompose to its glycone and aglycone. Haznedaroglu et al. [147] have optimized the parameters such as temperature, extraction time, and flow rate. Temperature and extraction time were found as the most effective parameters for TP and total flavonoids while extraction time and flow rate for anthocyanin contents. In addition, temperature and time were the leading parameters for the effectiveness of extracts on tyrosinase inhibition.

The optimal extraction temperature and time for glycosides in SBWE are shown in Figure 7. The optimal extraction temperature of organic acids is between 100 and $160{ }^{\circ} \mathrm{C}$, and the time is between 20 and $60 \mathrm{~min}$. 
Table 4. Subcritical water extraction of glycosides.

\begin{tabular}{|c|c|c|c|c|c|c|c|}
\hline Samples & $\begin{array}{l}\text { Medicinal } \\
\text { Parts }\end{array}$ & $\begin{array}{l}\text { Compounds } \\
\text { Extracted }\end{array}$ & $\begin{array}{l}\text { Extracts } \\
\text { Activity }\end{array}$ & $\begin{array}{l}\text { Extraction } \\
\text { Conditions }\end{array}$ & $\begin{array}{l}\text { Analytical } \\
\text { Methods }\end{array}$ & $\begin{array}{l}\text { Other Extraction } \\
\text { Methods (Solvent, } \\
\text { Ratios of Yields) }\end{array}$ & Ref. \\
\hline $\begin{array}{c}\text { Phaleria } \\
\text { macrocarpa }\end{array}$ & fruits & mangiferin & & $\begin{array}{c}323-423 \mathrm{~K} \\
1-7 \mathrm{~h} \\
0.7-4.0 \mathrm{Mpa}\end{array}$ & HPLC, LC-MS & $\begin{array}{c}\text { convention (water } \\
69.6 \% \text {; ethanol } 34.1 \% \text {; } \\
\text { methanol } 108 \% \text { ), HRE } \\
\text { (water } 85.7 \% \text {; ethanol } \\
60.8 \% \text {; methanol } 115 \% \text { ), } \\
\text { Soxhlet (water } 86.1 \% \text {; } \\
\text { ethanol } 55.8 \% \text {; } \\
\text { methanol } 113 \% \\
\text { methanol) }\end{array}$ & [36] \\
\hline $\begin{array}{l}\text { Punica } \\
\text { granatum } \mathrm{L} .\end{array}$ & $\begin{array}{l}\text { pomegranate } \\
\text { seed }\end{array}$ & $\begin{array}{l}\text { TP, kaempferol } \\
\text {-3-O-rutinoside }\end{array}$ & $\begin{array}{c}\text { antioxidant } \\
\text { (DPPH, ABTS) }\end{array}$ & $\begin{array}{l}80-280{ }^{\circ} \mathrm{C}, \\
5-120 \mathrm{~min} \\
1: 10- \\
1: 50 \mathrm{~g} / \mathrm{mL} \\
6.0 \mathrm{Mpa}\end{array}$ & $\begin{array}{c}\text { HPLC-DAD, } \\
\text { UV, } \\
\text { HPLC-ABTS }^{+}\end{array}$ & $\begin{array}{c}\text { leaching (water } 40.6 \% \text {; } \\
\text { methanol } 79.7 \% \text {; } \\
\text { ethanol } 41.7 \% \text {; acetone } \\
45.5 \% \text { ), UAE } \\
\text { (water } 11.3 \% \text {; methanol } \\
\text { 20.6\%; ethanol } 18.9 \% \text {; } \\
\text { acetone } 15.2 \% \text { ), Soxhlet } \\
\text { (methanol } 71.4 \% \text {; } \\
\text { acetone } 39.7 \% \text { ) }\end{array}$ & [144] \\
\hline $\begin{array}{c}\text { Teucrium } \\
\text { montanum L. }\end{array}$ & aerial parts & $\begin{array}{c}\text { rutin, naringin, } \\
\text { epicatechin, } \\
\text { etc. }\end{array}$ & $\begin{array}{c}\text { antioxidant } \\
\text { (DPPH, FRAP) }\end{array}$ & $\begin{array}{l}60-200{ }^{\circ} \mathrm{C}, \\
30 \mathrm{~min}, \\
1-10 \mathrm{Mpa}, \\
1: 10 \mathrm{~g} / \mathrm{mL}\end{array}$ & $\begin{array}{l}\text { HPLC-PDA, } \\
\text { UV }\end{array}$ & & [145] \\
\hline $\begin{array}{l}\text { Paeonia } \\
\text { lactiflora }\end{array}$ & root & $\begin{array}{c}\text { albiflorin, } \\
\text { paeoniflorin }\end{array}$ & & $\begin{array}{c}100-260{ }^{\circ} \mathrm{C} \\
10-60 \mathrm{~min}, 10- \\
40 \mathrm{~mL} / \mathrm{g}\end{array}$ & HPLC & $\begin{array}{l}\text { reflux (water 83.5\%), } \\
\text { UAE (ethanol } 77.8 \% \text { ) }\end{array}$ & [146] \\
\hline Morus nigra L. & fruits & $\begin{array}{c}\mathrm{TP}, \mathrm{TF} \\
\text { cyanidin } \\
\text { 3-glucoside, } \\
\text { etc. }\end{array}$ & $\begin{array}{l}\text { tyrosinase } \\
\text { inhibitory } \\
\text { activity }\end{array}$ & $\begin{array}{c}40-80{ }^{\circ} \mathrm{C}, \\
20-60 \mathrm{~min}, \\
2-6 \mathrm{~mL} / \mathrm{min}, \\
15 \mathrm{Mpa}\end{array}$ & $\begin{array}{l}\text { UPLC-DAD- } \\
\text { ESI-MS/MS }\end{array}$ & $\begin{array}{c}\text { shaker (ethanol:water } \\
116 \% \text { ), UAE } \\
\text { (ethanol:water:TFA } \\
134 \% \text { ) }\end{array}$ & [147] \\
\hline $\begin{array}{l}\text { Stevia } \\
\text { rebaudiana }\end{array}$ & leaves & $\begin{array}{r}\mathrm{TP}, \text { stevioside, } \\
\text { rebaudioside A }\end{array}$ & $\begin{array}{l}\text { antioxidants } \\
\text { (DPPH) }\end{array}$ & $\begin{array}{c}100-150{ }^{\circ} \mathrm{C}, \\
30-60 \mathrm{~min}, \\
23 \mathrm{Mpa}, \\
1: 10 \mathrm{~g} / \mathrm{mL}\end{array}$ & HPLC-UV, UV & & [148] \\
\hline $\begin{array}{c}\text { Erigeron } \\
\text { breviscapus }\end{array}$ & whole parts & $\begin{array}{l}\text { scutellarin, } 20 \\
\text { inorganic } \\
\text { elements, etc. }\end{array}$ & $\begin{array}{l}\text { antioxidant } \\
\text { (DPPH) }\end{array}$ & $\begin{array}{c}120-140{ }^{\circ} \mathrm{C}, \\
5-15 \mathrm{~min}, \\
150-420 \mathrm{um}\end{array}$ & $\begin{array}{c}\text { HPLC, } \\
\text { HPLC-MS }\end{array}$ & $\begin{array}{l}\text { reflux (methanol } 86.1 \% \\
\text { ethanol } 84.8 \% \text { ) }\end{array}$ & [149] \\
\hline $\begin{array}{l}\text { Mangifera } \\
\text { indica L. }\end{array}$ & leaves & $\begin{array}{l}\text { quercetin3-d- } \\
\text { glucoside, } \\
\text { mangiferin }\end{array}$ & $\begin{array}{l}\text { antioxidant } \\
\text { (DPPH) }\end{array}$ & $\begin{array}{l}100{ }^{\circ} \mathrm{C}, 4 \mathrm{Mpa}, \\
10 \mathrm{~g} / \mathrm{min}, 3 \mathrm{~h}\end{array}$ & UV, HPLC & $\begin{array}{c}\mathrm{SCCO}_{2}(20 \% \text { methanol } \\
18.7 \%)\end{array}$ & [150] \\
\hline $\begin{array}{c}\text { Crocus satious } \\
\text { L. }\end{array}$ & stigmas & $\begin{array}{l}\text { picrocrocin, } \\
\text { safranal, crocin }\end{array}$ & & $\begin{array}{l}5-15 \mathrm{~min}, \\
105-125^{\circ} \mathrm{C}\end{array}$ & $\begin{array}{l}\text { GC-MS, UV, } \\
\text { HPLC }\end{array}$ & & [151] \\
\hline $\begin{array}{c}\text { Glycyrrhiza } \\
\text { uralensis Fisch }\end{array}$ & $\begin{array}{l}\text { licorice } \\
\text { root }\end{array}$ & $\begin{array}{l}\text { TP, } \\
\text { glycyrrhetic } \\
\text { acid, } \\
\text { glycyrrhizin, } \\
\text { liquiritin }\end{array}$ & $\begin{array}{l}\text { antioxidant } \\
\text { (DPPH, } \\
\text { reducing } \\
\text { power) }\end{array}$ & $\begin{array}{c}50-300{ }^{\circ} \mathrm{C}, \\
10-60 \mathrm{~min}, \\
0.002-5 \mathrm{Mpa}\end{array}$ & HPLC, UV-Vis & & [152] \\
\hline
\end{tabular}

\subsection{Carbohydrates}

Carbohydrates is a very common term that include sugars, starch, and cellulose, which are an important class of organic compounds widely distributed in nature. The saccharides are divided into four groups: monosaccharides, disaccharides, oligosaccharides, and polysaccharides. As shown in Table 5, carbohydrates extracted by SBWE possess antioxidants [153], antimitotic [154], and growth inhibitory effects [55]. 
Table 5. Subcritical water extraction of carbohydrates.

\begin{tabular}{|c|c|c|c|c|c|c|c|}
\hline Samples & $\begin{array}{l}\text { Medicinal } \\
\text { Parts }\end{array}$ & $\begin{array}{l}\text { Compounds } \\
\text { Extracted }\end{array}$ & Activity/Mixtures & $\begin{array}{l}\text { Extraction } \\
\text { Conditions }\end{array}$ & $\begin{array}{l}\text { Analytical } \\
\text { Methods }\end{array}$ & $\begin{array}{l}\text { Other Extraction } \\
\text { Methods (Solvent, } \\
\text { Ratios of Yields) }\end{array}$ & Ref. \\
\hline $\begin{array}{l}\text { Lycium } \\
\text { barbarum }\end{array}$ & berries & $\begin{array}{l}\text { total sugar } \\
\text { content }\end{array}$ & $\begin{array}{c}\text { antioxidant } \\
\text { (FRAP, TEAC), } \\
\text { immunomodu- } \\
\text { latory }\end{array}$ & $\begin{array}{c}1: 30 \mathrm{~g} / \mathrm{mL}, \\
110{ }^{\circ} \mathrm{C}, 5 \mathrm{Mpa}\end{array}$ & HPGPC & $\begin{array}{l}\text { HWE (water } 71.5 \% \text { ), } \\
\text { UAE (water } 89.9 \% \text { ), } \\
\text { UWE (water } 132 \% \text { ) }\end{array}$ & [11] \\
\hline sunflower & $\begin{array}{l}\text { sunflower } \\
\text { heads }\end{array}$ & $\begin{array}{l}\text { galacturonic } \\
\text { acid, pectin }\end{array}$ & & $\begin{array}{c}10-50 \mathrm{~min}, \\
2-8 \mathrm{~mL} / \mathrm{g}, \\
100-140{ }^{\circ} \mathrm{C} \\
0.2-1 \mathrm{Mpa}\end{array}$ & $\begin{array}{l}\text { TG/TGA, DSC, } \\
\text { UV-vis, FTIR, } \\
\text { HPSEC, NMR }\end{array}$ & & [40] \\
\hline $\begin{array}{c}\text { Aronia } \\
\text { melanocarpa }\end{array}$ & $\begin{array}{l}\text { chokeberry } \\
\text { stems }\end{array}$ & $\begin{array}{c}1 \text { amino acid, } 8 \\
\text { alcohols, } 11 \\
\text { sugars, } 2 \text { fatty } \\
\text { acids, etc. }\end{array}$ & $\begin{array}{l}\text { antioxidant } \\
\text { (DPPH), } \\
\text { enzyme } \\
\text { inhibitory } \\
\text { activity }\end{array}$ & $\begin{array}{c}130{ }^{\circ} \mathrm{C}, \\
3.5 \mathrm{Mpa}, \\
20 \mathrm{~min}, \\
1: 20 \mathrm{~g} / \mathrm{mL}\end{array}$ & GC-MS & & [49] \\
\hline Lentinus edodes & fruit bodies & $\begin{array}{c}\text { hetero- } \\
\text { polysaccharides, } \\
\text { xylose, } \\
\text { mannose, etc. }\end{array}$ & $\begin{array}{c}\text { antioxidant } \\
\text { (OH., DPPH, } \\
\text { ABTS) }\end{array}$ & $\begin{array}{c}120-160{ }^{\circ} \mathrm{C}, \\
30-50 \mathrm{~min}, \\
0.033-0.05 \mathrm{~g} / \mathrm{mL}\end{array}$ & $\begin{array}{l}\text { UV-vis, SEM, } \\
\text { GC, GPC, } \\
\text { FT-IR }\end{array}$ & & {$[54]$} \\
\hline Lentinus edodes & fruit bodies & $\begin{array}{c}\text { 1-rhamnose, } \\
\text { d-arabinose, } \\
\text { d-xylose, } \\
\text { d-mannose }\end{array}$ & $\begin{array}{l}\text { antioxidant } \\
\text { (ABTS), } \\
\text { growth } \\
\text { inhibitory } \\
\text { effect }\end{array}$ & $\begin{array}{l}100-150{ }^{\circ} \mathrm{C}, \\
10-30 \mathrm{~min}, \\
5 \mathrm{Mpa}\end{array}$ & $\begin{array}{l}\text { FT-IR, UV-Vis, } \\
\text { AFM, GC, HP } \\
\text { SEC-MALLS }\end{array}$ & & [55] \\
\hline Lentinus edodes & fruit bodies & $\begin{array}{l}\text { polysaccharides, } \\
\text { rhamnose, } \\
\text { arabinose, } \\
\text { xylose, etc. }\end{array}$ & $\begin{array}{l}\text { antioxidant } \\
\text { (DPPH, } \\
\text { reducing } \\
\text { power) }\end{array}$ & $\begin{array}{c}140{ }^{\circ} \mathrm{C}, 40 \mathrm{~min}, \\
1: 25 \mathrm{~g} / \mathrm{mL}, \\
5 \mathrm{Mpa}\end{array}$ & $\begin{array}{l}\text { GC, FT-IR, } \\
\text { AFM, SEM }\end{array}$ & & [56] \\
\hline Lentinula edodes & fruit bodies & $\begin{array}{l}\text { TCC, total } \\
\beta \text {-glucan, } \\
\text { chitin }\end{array}$ & $\begin{array}{l}\text { HMGCR, } \\
\text { immuno- } \\
\text { modulatory }\end{array}$ & $\begin{array}{c}200{ }^{\circ} \mathrm{C} \\
11.7 \mathrm{Mpa}, \\
15-60 \mathrm{~min}\end{array}$ & $\begin{array}{c}\text { GC-MS, } \\
\text { HPSEC, NMR }\end{array}$ & $\begin{array}{l}\text { UAE (water } 65.2 \% \text { ), } \\
\text { HWE (water } 32.3 \% \text { ), } \\
\text { SPE (water } 33.0 \% \text { ) }\end{array}$ & [57] \\
\hline Grifola frondosa & fruit bodies & $\begin{array}{l}\text { total polysac- } \\
\text { charide, total } \\
\text { protein }\end{array}$ & $\begin{array}{l}\text { antioxidant } \\
\text { (DPPH, } \\
\text { reducing } \\
\text { power) }\end{array}$ & $\begin{array}{c}100-230{ }^{\circ} \mathrm{C}, \\
2-4 \mathrm{~min}, \\
20-100 \mathrm{mesh}, \\
5 \mathrm{Mpa}\end{array}$ & FT-IR, SEM & HWE (water 87.8\%) & [58] \\
\hline $\begin{array}{c}\text { Sagittaria } \\
\text { sagittifolia } \mathrm{L} .\end{array}$ & fruit bodies & polysaccharides & $\begin{array}{c}\text { antioxidant } \\
\text { (DPPH, ABTS, } \\
\text { reducing } \\
\text { power,) }\end{array}$ & $\begin{array}{c}150-190{ }^{\circ} \mathrm{C}, \\
12-20 \mathrm{~min}, \\
1: 20- \\
1: 40 \mathrm{~g} / \mathrm{mL}, \\
\mathrm{pH} 7-9\end{array}$ & $\begin{array}{l}\text { FT-IR, } 1 \mathrm{H} \text { and } \\
13 \mathrm{C} \text { NMR, UV }\end{array}$ & HWE (water 55.8\%) & [59] \\
\hline $\begin{array}{c}\text { Sagittaria } \\
\text { sagittifolia } \mathrm{L} .\end{array}$ & fruit bodies & $\begin{array}{c}\text { 1-rhamnose, } \\
\text { d-arabinose, } \\
\text { d-xylose, } \\
\text { d-mannose }\end{array}$ & $\begin{array}{l}\text { antioxidant, } \\
\text { immuno- } \\
\text { modulatory }\end{array}$ & $170^{\circ} \mathrm{C}, 16 \mathrm{~min}$, & $\begin{array}{c}\text { HPLC, GC, } \\
\text { SEM, IR, AFM, } \\
\text { HPSEC- } \\
\text { MALLS }\end{array}$ & $\begin{array}{l}\text { HWE (water } 75.6 \% \text { ); } \\
\text { UAE (water } 96.1 \% \text { ) }\end{array}$ & [60] \\
\hline $\begin{array}{c}\text { Sagittaria } \\
\text { sagittifolia } \mathrm{L} .\end{array}$ & fruit bodies & $\begin{array}{c}\alpha \text {-pyranose } \\
\text { polysaccha- } \\
\text { ride, } \\
\beta \text {-pyranose } \\
\text { polysaccharide }\end{array}$ & $\begin{array}{l}\text { immuno- } \\
\text { stimulatory }\end{array}$ & $\begin{array}{c}1 \mathrm{Mpa}, \\
\mathrm{pH} 7,170^{\circ} \mathrm{C}, \\
16 \mathrm{~min}, \\
30: 1 \mathrm{~mL} / \mathrm{g}\end{array}$ & $\begin{array}{l}\text { IR, GC-FID, } \\
\text { UV, HPSEC, } \\
\text { AFM }\end{array}$ & & [61] \\
\hline $\begin{array}{l}\text { Cordyceps } \\
\text { militaris }\end{array}$ & fruit bodies & $\begin{array}{l}\text { total sugars, } \\
\text { protein and } \\
\text { uronic acid }\end{array}$ & & $\begin{array}{c}180{ }^{\circ} \mathrm{C}, 13 \mathrm{~min}, \\
\mathrm{pH}=8, \\
21 \mathrm{~mL} / \mathrm{g}\end{array}$ & $\begin{array}{l}\text { IR, GC, AFM, } \\
\text { GPC-MALLS }\end{array}$ & & [62] \\
\hline wheat & bran & $\begin{array}{l}\text { monosaccharide, } \\
\text { etc. }\end{array}$ & $\begin{array}{l}\text { antioxidants } \\
\text { (DPPH) }\end{array}$ & $\begin{array}{l}160-180{ }^{\circ} \mathrm{C} \\
5-60 \mathrm{~min}\end{array}$ & $\begin{array}{l}\text { HPAEC-PAD, } \\
\text { SEC }\end{array}$ & & {$[153]$} \\
\hline $\begin{array}{l}\text { Saccharina } \\
\text { japonica }\end{array}$ & & $\begin{array}{c}\text { fucoidan, } \\
\text { fucose, glucose, } \\
\text { galactose, } \\
\text { mannose, etc. }\end{array}$ & $\begin{array}{l}\text { antioxidant, } \\
\text { antimitotic } \\
\text { anti- } \\
\text { proliferative }\end{array}$ & $\begin{array}{l}100-180{ }^{\circ} \mathrm{C} \\
5-15 \mathrm{~min} \\
\text { 2-8 Mpa }\end{array}$ & $\begin{array}{l}\text { FTIR, TGA, } \\
\text { UV-Vis }\end{array}$ & $\begin{array}{c}\text { convention }(0.05 \mathrm{M} \\
\mathrm{HCl} 100 \%)\end{array}$ & {$[154]$} \\
\hline $\begin{array}{c}\text { Citrus grandis } \\
\text { L. }\end{array}$ & pomelo peel & pectin & & $\begin{array}{l}90-120{ }^{\circ} \mathrm{C} \\
3-10 \mathrm{Mpa}\end{array}$ & $\begin{array}{l}\text { HPSEC- } \\
\text { MALLS }\end{array}$ & & {$[155]$} \\
\hline
\end{tabular}


Table 5. Cont.

\begin{tabular}{|c|c|c|c|c|c|c|c|}
\hline Samples & $\begin{array}{l}\text { Medicinal } \\
\text { Parts }\end{array}$ & $\begin{array}{l}\text { Compounds } \\
\text { Extracted }\end{array}$ & Activity/Mixtures & $\begin{array}{c}\text { Extraction } \\
\text { Conditions }\end{array}$ & $\begin{array}{l}\text { Analytical } \\
\text { Methods }\end{array}$ & $\begin{array}{l}\text { Other Extraction } \\
\text { Methods (Solvent, } \\
\text { Ratios of Yields) }\end{array}$ & Ref. \\
\hline $\begin{array}{c}\text { Theobroma cacao } \\
\text { L. }\end{array}$ & $\begin{array}{l}\text { cacao pod } \\
\text { husks }\end{array}$ & $\begin{array}{c}\text { xylose, } \\
\text { arabinose, etc. }\end{array}$ & & $\begin{array}{c}121^{\circ} \mathrm{C}, 30 \mathrm{~min}, \\
10.3 \mathrm{Mpa}\end{array}$ & $\begin{array}{l}\text { FT-IR, GC-FID, } \\
\text { SEM }\end{array}$ & $\begin{array}{c}\text { convention (4\% citric } \\
\text { acid } 76.1 \%)\end{array}$ & [156] \\
\hline $\begin{array}{l}\text { Kappaphycus } \\
\text { alvarezii. A }\end{array}$ & & $\begin{array}{l}\text { k-carrageenan, } \\
\text { glucose, 3,6- } \\
\text { anhydrogalactose, } \\
\text { etc. }\end{array}$ & $\begin{array}{c}\text { antioxidant } \\
\text { (DPPH, ABTS) }\end{array}$ & $\begin{array}{c}60-180^{\circ} \mathrm{C} \\
5 \mathrm{Mpa}, 5 \mathrm{~min}\end{array}$ & $\begin{array}{l}\text { FTIR, TGA, } \\
\text { XRD }\end{array}$ & $\begin{array}{c}\text { convention (water } \\
94.3 \% \text {; water with IL } \\
101 \% \text { ) }\end{array}$ & [157] \\
\hline $\begin{array}{l}\text { Pseuderanthemum } \\
\text { palatiferum }\end{array}$ & leaves & $\begin{array}{l}\text { TCC, monosac- } \\
\text { charides }\end{array}$ & $\begin{array}{l}\text { anticoagulant, } \\
\text { antioxidant }\end{array}$ & $\begin{array}{c}150-200{ }^{\circ} \mathrm{C} \\
5-10 \mathrm{~mL} / \mathrm{min}\end{array}$ & $\begin{array}{l}\text { HPLC, GPC, } \\
\text { NMR, UV }\end{array}$ & $\begin{array}{c}\text { convention }(0.1 \mathrm{M} \\
\mathrm{NaOH} 48.8 \%)\end{array}$ & [158] \\
\hline wheat & bran & $\begin{array}{l}\text { TCC, reducing } \\
\text { sugar, } \\
\text { arabinose, } \\
\text { xylose, etc. } \\
\end{array}$ & $\begin{array}{l}\text { antioxidant, } \\
\alpha \text {-amylase } \\
\text { inhibitory }\end{array}$ & $\begin{array}{c}140^{\circ} \mathrm{C}, 5 \mathrm{Mpa}, \\
30 \mathrm{~min}\end{array}$ & $\begin{array}{l}\text { SEC-MALLS, } \\
\text { FT-IR, DLS, } \\
\text { DSC, UV }\end{array}$ & $\begin{array}{c}\text { SBWE (water with } \\
\text { citric acid 97.6\%); UWE } \\
\text { (water with citric acid } \\
103 \% \text { ) }\end{array}$ & [159] \\
\hline $\begin{array}{l}\text { Lycium } \\
\text { barbarum } \mathrm{L} \text {. }\end{array}$ & fruits & polysaccharides & $\begin{array}{l}\text { antioxidant } \\
(\mathrm{O} 2 \cdot, \mathrm{OH} \cdot, \\
\mathrm{DPPH})\end{array}$ & $\begin{array}{c}5 \mathrm{Mpa}, \\
25 \mathrm{~mL} / \mathrm{g} \\
110^{\circ} \mathrm{C}, 1 \mathrm{~h}\end{array}$ & UV & $\begin{array}{l}\text { HWE (water } 86.2 \% \text { ); } \\
\text { UAE (water74.9\%); } \\
\text { UWE (water } 111 \% \text { ) }\end{array}$ & [160] \\
\hline $\begin{array}{c}\text { Cocos nucifera } \\
\text { L. }\end{array}$ & $\begin{array}{l}\text { defatted } \\
\text { coconut }\end{array}$ & $\begin{array}{c}\text { mannose, } \\
\text { galactosamine, } \\
\text { xylose, } \\
\text { rhamnose, etc. }\end{array}$ & $\begin{array}{l}\text { antioxidant, } \\
\text { hypogly- } \\
\text { caemic, } \\
\text { adsorption }\end{array}$ & $\begin{array}{c}1: 10- \\
1: 50 \mathrm{~g} / \mathrm{mL}, \\
10-50 \mathrm{~min}, \\
120-200^{\circ} \mathrm{C} \\
20-100 \mathrm{mesh}\end{array}$ & $\begin{array}{l}\text { HPLC, XRD, } \\
\text { TGA, DTGA, } \\
\text { SEM, FT-IR }\end{array}$ & & [161] \\
\hline okara & & $\begin{array}{l}\text { polysaccharides, } \\
\text { TP, TF }\end{array}$ & $\begin{array}{c}\text { antioxidant } \\
\text { (ABTS, DPPH) }\end{array}$ & $\begin{array}{c}1: 30 \mathrm{~g} / \mathrm{mL} \\
160-230{ }^{\circ} \mathrm{C} \\
10 \mathrm{~min}\end{array}$ & UV & & [162] \\
\hline $\begin{array}{l}\text { Saccharina } \\
\text { japonica }\end{array}$ & & $\begin{array}{l}\text { polysaccharide, } \\
\text { fucoidan, } \\
\text { alginate }\end{array}$ & $\begin{array}{c}\text { antioxidant } \\
\text { (ABTS, DPPH, } \\
\text { FRAP) }\end{array}$ & $\begin{array}{c}100-150{ }^{\circ} \mathrm{C} \\
1-5 \mathrm{Mpa} \\
1: 30-1: 50 \mathrm{~g} / \mathrm{mL}\end{array}$ & $\begin{array}{l}\text { IR, DSC, TGA, } \\
{ }^{1} \text { HNMR, } \\
\text { HPLC, } \\
\text { HPSEC-ELSD }\end{array}$ & & [163] \\
\hline Passiflora edulis & fruit peel & $\begin{array}{l}\text { pectic polysac- } \\
\text { charide, } \\
\text { mannose, } \\
\text { glucose, etc. }\end{array}$ & $\begin{array}{l}\text { antioxidant } \\
\text { (DPPH) }\end{array}$ & $\begin{array}{c}100-160{ }^{\circ} \mathrm{C} \\
5.64-7.94 \mathrm{~min}, \\
10-30 \% \text { ethanol }\end{array}$ & $\begin{array}{l}\text { HPLC, UV, } \\
\text { viscometer }\end{array}$ & & [164] \\
\hline $\begin{array}{l}\text { Chlorella } \\
\text { vulgaris, } \\
\text { Sargassum } \\
\text { vulgare, } \\
\text { Sargassum } \\
\text { muticum, } \\
\text { Porphyra spp., } \\
\text { Cystoseira } \\
\text { abies-marina, } \\
\text { Undaria } \\
\text { pinnatifida and } \\
\text { Halopitys } \\
\text { incurvus, } \\
\text { Rosmarinus } \\
\text { officinalis L., } \\
\text { Thymus } \\
\text { vulgaris, } \\
\text { Verbena } \\
\text { officinalis }\end{array}$ & $\begin{array}{l}\text { microalgae, } \\
\text { algae, leaves }\end{array}$ & $\begin{array}{l}\text { sugar, TP, } \\
\text { melanoidins }\end{array}$ & $\begin{array}{l}\text { antioxidant } \\
\left(\mathrm{ABTS}, \mathrm{O}_{2}^{-}\right)\end{array}$ & $\begin{array}{c}100-200^{\circ} \mathrm{C} \\
20 \mathrm{~min}, 10 \mathrm{Mpa}\end{array}$ & UV & & [165] \\
\hline rice bran & bran & $\begin{array}{l}\text { protein, TCC, } \\
\text { TP }\end{array}$ & $\begin{array}{l}\text { antioxidant } \\
\text { (DPPH) }\end{array}$ & $\begin{array}{c}120-250^{\circ} \mathrm{C}, 0.5- \\
5 \mathrm{~mL} / \mathrm{min}\end{array}$ & $\mathrm{UV}$, UV-Vis & & [166] \\
\hline $\begin{array}{c}\text { Nizamuddinia } \\
\text { zanardinii }\end{array}$ & & $\begin{array}{l}\text { TCC, } \\
\text { rhamnose, } \\
\text { xylose, } \\
\text { arabinose, } \\
\text { fucoidan, } \\
\text { fucose }\end{array}$ & $\begin{array}{l}\text { antioxidant, } \\
\text { anticancer, } \\
\text { macrophage, } \\
\text { etc. }\end{array}$ & $\begin{array}{c}425 \mathrm{rpm}, \\
10-30 \mathrm{~min}, \\
90-150^{\circ} \mathrm{C}, \\
0-40 \mathrm{~mL} / \mathrm{g}, \\
0.75 \mathrm{Mpa}, 1500 \\
\text { W }\end{array}$ & $\begin{array}{l}\text { FT-IR, GC-MS, } \\
\text { SEM, UV, } \\
\text { HPSEC- } \\
\text { MALLS-RI }\end{array}$ & & [167] \\
\hline
\end{tabular}


Table 5. Cont.

\begin{tabular}{|c|c|c|c|c|c|c|c|}
\hline Samples & $\begin{array}{l}\text { Medicinal } \\
\text { Parts }\end{array}$ & $\begin{array}{l}\text { Compounds } \\
\text { Extracted }\end{array}$ & Activity/Mixtures & $\begin{array}{l}\text { Extraction } \\
\text { Conditions }\end{array}$ & $\begin{array}{l}\text { Analytical } \\
\text { Methods }\end{array}$ & $\begin{array}{l}\text { Other Extraction } \\
\text { Methods (Solvent, } \\
\text { Ratios of Yields) }\end{array}$ & Ref. \\
\hline $\begin{array}{l}\text { Dendrobiumnobile } \\
\text { Lindl. }\end{array}$ & stems & $\begin{array}{c}\text { polysaccharide, } \\
\text { arabinose, } \\
\text { galactose, } \\
\text { glucose, etc. }\end{array}$ & $\begin{array}{c}\text { antioxidant } \\
(\mathrm{OH} \cdot, \text { ABTS) }\end{array}$ & $\begin{array}{l}0.5-1.5 \mathrm{Mpa}, \\
5-20 \mathrm{~min} \\
120-160{ }^{\circ} \mathrm{C} \\
1: 25 \mathrm{~g} / \mathrm{mL}\end{array}$ & $\begin{array}{l}\text { UV-vis, GPC, } \\
\text { HPLC, HPAEC }\end{array}$ & & [168] \\
\hline $\begin{array}{l}\text { Ecklonia } \\
\text { maxima }\end{array}$ & & $\begin{array}{l}\text { TP, polysaccha- } \\
\text { ride, sulphate, } \\
\text { and alginate }\end{array}$ & $\begin{array}{l}\text { antioxidant } \\
\text { (ABTS) }\end{array}$ & $\begin{array}{c}100-180{ }^{\circ} \mathrm{C} \\
\text { 5-30 min, } \\
10-50 \mathrm{~mL} / \mathrm{g} \\
4 \mathrm{Mpa}\end{array}$ & $\begin{array}{l}\text { UV, elemental } \\
\text { analysis, } \\
\text { ICP-MS }\end{array}$ & $\begin{array}{c}\text { convention }(70 \% \\
\text { ethanol } 0 \% ; 0.05 \mathrm{M} \mathrm{HCl} \\
20.1 \%)\end{array}$ & [169] \\
\hline Vitis vinifera & grape pomace & $\begin{array}{c}\text { glucose, } \\
\text { fructose, } \\
\text { galactose, } \\
\text { arabinose, } \\
\text { mannose, etc. }\end{array}$ & $\begin{array}{l}\text { antimicrobial, } \\
\text { antioxidant } \\
(\mathrm{DPPH})\end{array}$ & $\begin{array}{c}170-210^{\circ} \mathrm{C}, \\
10 \mathrm{Mpa}, \\
5-10 \mathrm{~mL} / \mathrm{min}\end{array}$ & HPLC, UV & & [170] \\
\hline $\begin{array}{l}\text { green coffee } \\
\text { beans }\end{array}$ & $\begin{array}{l}\text { spent coffee } \\
\text { grounds }\end{array}$ & $\begin{array}{l}\text { carbohydrates, } \mathrm{pH} \\
\text { nolics }\end{array}$ & $\begin{array}{l}\text { He- antioxidant, } \\
\text { antibacterial }\end{array}$ & $\begin{array}{c}150-220{ }^{\circ} \mathrm{C}, \\
7 \mathrm{Mpa}, \\
10 \mathrm{~mL} / \mathrm{min}\end{array}$ & HPLC, UV & & [171] \\
\hline $\begin{array}{l}\text { Tamarindus } \\
\text { indica }\end{array}$ & seed & TP, xyloglucan & $\begin{array}{l}\text { antioxidant } \\
\text { (DPPH) }\end{array}$ & $\begin{array}{l}100-200^{\circ} \mathrm{C}, \\
1: 20 \mathrm{~g} / \mathrm{mL}\end{array}$ & SEC, UV & $\begin{array}{c}\text { convention (water } \\
74.6 \% \text { ) }\end{array}$ & [172] \\
\hline Mentha arvensis & leaves & $\begin{array}{l}\text { carbohydrates, } \\
\text { apocynin }\end{array}$ & $\begin{array}{l}\text { antioxidant } \\
(\mathrm{DPPH})\end{array}$ & $\begin{array}{l}180-260{ }^{\circ} \mathrm{C}, \\
1: 20 \mathrm{~g} / \mathrm{mL}, \\
5 \mathrm{~min}\end{array}$ & $\begin{array}{l}\text { HPLC, GC-MS, } \\
\text { UV, }\end{array}$ & & [173] \\
\hline
\end{tabular}

Traditional extraction solvents often utilize alkaline solution [154], acidic solution [156], and water as extraction solvents [157]. Chun et al. [154] carried out SBWE from Pseuderanthemum palatiferum Radlk and co Mpared it with a conventional technique using $0.1 \mathrm{M}$ sodium hydroxide. Conjugates obtained from SBWE at $150{ }^{\circ} \mathrm{C}$ exhibited better anticoagulant activity than those found at $200^{\circ} \mathrm{C}$ and were co Mparable to that of the conventional technique. Villamiel et al. [156] and Zhang et al. [159] used citric acid solution to adjust the $\mathrm{pH}$ to 3.0, 5.0, respectively. Co Mpared to the conventional extraction with citric acid, SBWE can obtain higher pectin yield, higher galacturonic acid content and a higher degree of methyl esterification. Similarly, Chun et al. [158] and Goosen et al. [169] carried out SBWE and co Mpared it with conventional extraction using $0.05 \mathrm{M} \mathrm{HCl}$. They all found that SBWE efficiency was significantly higher co Mpared to the conventional methods.

SBWE and other three innovative technologies UAE, MAE, and supercritical fluid extraction (SFE) has been co Mpared or coupled. Duan et al. [56] have used UAE to successfully extract the chief ingredient polysaccharides from Lentinus edodes. A Box-Behnken design (BBD) was applied to optimize the UAE condition including extraction temperature, extraction time, liquid-to-solid ratio, and ultrasonic power. It was demonstrated that the UAE sample has better antioxidant activities co Mpared with other extraction methods (SBWE, UAE). Morales et al. [57] have found UAE or SBWE $\left(200{ }^{\circ} \mathrm{C}, 11.7 \mathrm{Mpa}\right)$ were more effective to obtain $\beta$-glucan enriched fractions from shiitake mushrooms. They revealed that a combination of UAE + SBWE extracts showed larger glucose levels and lower mannose and galactose residues than the other extractions, suggesting certain extraction specificity towards $\beta$-glucans. Moreover, the extracts obtained after combination of technologies partially retained their immunomodulatory properties, but they showed high hypocholesterolemic activities according to in vitro studies.

Sagittaria sagittifolia $\mathrm{L}$. is a healthy food source and a restorative for the adjuvant therapy of tuberculosis, night blindness, pancreatitis, diabetes, tracheitis, and urinary tract infections. Ma and his team [35] have conducted continuous work on the characterization, antioxidant, and immunological activities of SBWE extracts from S. sagittifolia $\mathrm{L}$. The extracts' structural features were elucidated using high performance liquid chromatography (HPLC), gas chromatography (GC), scanning electron microscopy (SEM), infrared spectroscopy (IR), atomic force microscopy (AFM), zeta potential, and Congo red methods. 
Extracted polysaccharides exhibited stronger antioxidant activity in vitro and more potent immunomodulatory activity. Therefore, the polysaccharides extracted from $S$. sagittifolia L. with SBWE have the potential to be used as immunoreactive agent in medicine and functional foods. Plaza et al. have found the Maillard, caramelization and thermoxidation, and Folin reaction from microalgae, macroalgae, and rosemary samples. It is the first time the neoformation of antioxidants during SWE extraction of different natural products is verified [165]. Sometimes the high operation temperatures may generate new bioactive compounds, and SBWE is a very promising technique for obtaining bioactive compounds from natural products. As shown in Figure 9, the optimal extraction temperature of organic acids is between 120 and $180{ }^{\circ} \mathrm{C}$, and the time is between 10 and $40 \mathrm{~min}$.

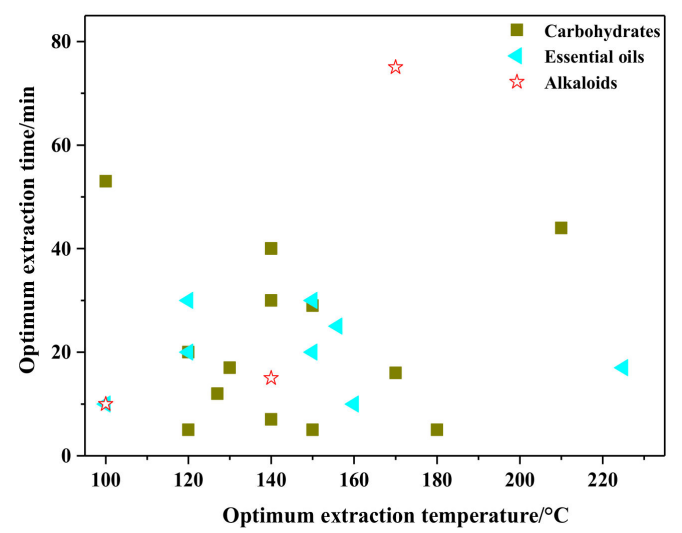

Figure 9. Optimum extraction conditions for carbohydrates, essential oils, and alkaloids.

\subsection{Essential Oils}

Essential oils, also called volatile oils or ethereal oils, refer to the volatile chemical compounds derived from plants. Essential oils often consist of the parts of the flowers, leaves, wood, bark, root, seeds, or peel. Ethereal oils are usually lipophilic and easily soluble in oils, which enables them to easily penetrate the skin and enter the body through the rich capillaries under the subcutaneous fat. Essential oils have been used in folk medicine in ancient China, Egypt, Arabia, and Greece throughout centuries. Therefore, some researches have attempted to utilize SBWE as a green solvent to extract essential oils from plants, and recent researches are illustrated in Table $6[12,22,28,174-206]$. The most common essential oils are extracted by distillation [174,176,180,181], $n$-hexane [174] or supercritical carbon dioxide [181], or dichloromethane [181].

Coriander (Coriandrum sativum L.) seeds contain an essential oil (up to 1\%) and are increasingly used as condiment in the food industry. Eikani et al. [174] and Zeković et al. $[175,181]$ have extracted and isolated the essential oil by SBWE. Extraction temperatures $\left(100,125,150\right.$, and $\left.175^{\circ} \mathrm{C}\right)$, mean particle sizes $(0.25,0.50$, and $1 \mathrm{~mm})$, and water flow rates $(1,2$, and $4 \mathrm{~mL} / \mathrm{min})$ were investigated by Eikani et al. Separation and identification of the components were carried out by GC-FID and GC-MS. They concluded that hydro distillation and Soxhlet extraction showed higher extraction efficiency, but the SBWE resulted in the essential oils more being concentrated in valuable oxygenated components. Zoran et al. also concluded that the SBWE process would have advantage in terms of time consumption since $20 \mathrm{~min}$ for this process was significantly lower than the $2 \mathrm{~h}, 4-5 \mathrm{~h}$, and $4 \mathrm{~h}$ required for herbal decoction, Soxhlet, and supercritical fluid extraction, respectively.

Most essential oils extracted by SBWE have no research on the activities, except for Ma et al. [176], who extracted essential oils from K. galangal using ultrasound-enhanced SBWE and investigated the antioxidant ability of the essential oils. The effects of temperature, extraction time, pressure, ultrasonic power density, and frequency on the extraction yield were investigated. The antioxidant activity of the essential oils was evaluated by the assays of the 2,2-diphenyl-1-picryl-hydrazyl (DPPH) scavenging ability and the superoxide anion radical scavenging activity. The result demonstrated that antioxidant effects of UAE 
extracted essential oil were better than that of herbal decoction and SBWE. As shown in Figure 9, the optimal extraction temperature of essential oils is between 120 and $160{ }^{\circ} \mathrm{C}$, and the time is between 10 and $35 \mathrm{~min}$.

Table 6. SBWE of essential oils, alkaloids, quinones, terpenes, lignans, and steroids.

\begin{tabular}{|c|c|c|c|c|c|c|}
\hline Samples & $\begin{array}{l}\text { Medicinal } \\
\text { Parts }\end{array}$ & Compounds Extracted & $\begin{array}{l}\text { Extraction } \\
\text { Conditions }\end{array}$ & Methods & $\begin{array}{l}\text { Other Extraction } \\
\text { Methods (Solvent, } \\
\text { Ratios of Yields) }\end{array}$ & Ref. \\
\hline \multicolumn{7}{|c|}{ Essential oils } \\
\hline $\begin{array}{c}\text { Thymbra spicata } \\
\text { L. }\end{array}$ & leaves & $\begin{array}{l}\alpha \text {-thujene, } \alpha \text {-pinene, } \\
\text { terpinen-4-ol, } \\
\text { p-cymene, } \gamma \text {-terpinene, } \\
\text { 1-carvone, thymol, } \\
\text { carvacrol, etc. }\end{array}$ & $\begin{array}{l}100-175^{\circ} \mathrm{C}, \\
1-3 \mathrm{~mL} / \mathrm{min} \\
2-9 \mathrm{Mpa} \\
30 \mathrm{~min}\end{array}$ & $\begin{array}{l}\text { GC-TOF/MS, } \\
\text { GC-FID }\end{array}$ & & [12] \\
\hline $\begin{array}{l}\text { Aquilaria } \\
\text { malaccensis }\end{array}$ & leaves & $\begin{array}{c}\text { butanal, } \\
\text { cyclopentanone, } \\
\text { acetoxyacetone, } \\
\text { benzaldehyde, } \\
\text { acetophenone, creosol, } \\
\text { etc. }\end{array}$ & $\begin{array}{c}100-271^{\circ} \mathrm{C}, \\
1-34 \mathrm{~min}, \\
0.08-0.22 \mathrm{~g} / \mathrm{mL}\end{array}$ & $\begin{array}{l}\text { GC-MS, SEM, } \\
\text { FT-IR }\end{array}$ & $\operatorname{HD}(95.4 \%)$ & [22] \\
\hline $\begin{array}{c}\text { Mentha piperita } \\
\text { L. }\end{array}$ & $\begin{array}{l}\text { peppermint } \\
\text { leaves }\end{array}$ & $\begin{array}{l}\mathrm{TP}, \text { menthone, menthol, } \\
\text { eriocitrin, etc. }\end{array}$ & $\begin{array}{l}40-160{ }^{\circ} \mathrm{C} \\
10.3 \mathrm{Mpa}, \\
1-30 \mathrm{~min}\end{array}$ & $\begin{array}{l}\text { GC-MS, FID, } \\
\text { HPLC }\end{array}$ & $\begin{array}{c}\text { convention } \\
\text { (methanol 53.2\%) }\end{array}$ & [28] \\
\hline $\begin{array}{l}\text { Coriandrum } \\
\text { satioum L. }\end{array}$ & $\begin{array}{l}\text { coriander } \\
\text { seeds }\end{array}$ & $\begin{array}{l}\text { thujene, sabinene, } \\
\text { pinene, myrcene, } \\
\text { cymene, limonene, } \\
\text { ocimene, terpinene, } \\
\text { terpinolene, etc. }\end{array}$ & $\begin{array}{c}100-175^{\circ} \mathrm{C}, \\
1-4 \mathrm{~mL} / \mathrm{min}, \\
0.25-1 \mathrm{~mm}, \\
2 \mathrm{Mpa}, 20 \mathrm{~min}\end{array}$ & $\begin{array}{l}\text { GC-FID, } \\
\text { GC-MS }\end{array}$ & $\begin{array}{l}\text { HD (1.54-fold), } \\
\text { Soxhlet (hexane } \\
\text { 1.4-fold) }\end{array}$ & [174] \\
\hline $\begin{array}{l}\text { Coriandrum } \\
\text { satioum L. }\end{array}$ & $\begin{array}{l}\text { coriander } \\
\text { seeds }\end{array}$ & $\begin{array}{l}3,4- \\
\text { dimethoxycinnamic } \\
\text { acid, coumaric acid, } \\
\text { sinapic acid, cis-and } \\
\text { trans-linalooloxides, } \\
\text { linalool, etc. }\end{array}$ & $\begin{array}{c}100-200{ }^{\circ} \mathrm{C}, \\
\text { 10-30 min, } \\
\text { 3-9 Mpa }\end{array}$ & $\begin{array}{l}\text { HPLC-MS/MS, } \\
\text { GC-MS }\end{array}$ & & [175] \\
\hline $\begin{array}{l}\text { Kaempferia } \\
\text { galangal L. }\end{array}$ & rhizome & $\begin{array}{c}\text { ethyl-p- } \\
\text { methoxycinnamate, } \\
\text { d-limonene, eucalyptol, } \\
\text { tridecane, camphor, } \\
\text { borneol, tetradecane, } \\
\text { etc. }\end{array}$ & $\begin{array}{c}120{ }^{\circ} \mathrm{C}, 10 \mathrm{Mpa}, \\
30 \mathrm{~min}\end{array}$ & GC-MS & $\begin{array}{c}\mathrm{HD}(82.3 \%), \mathrm{UWE} \\
(100 \%)\end{array}$ & [176] \\
\hline Piper betle & leaves & $\begin{array}{l}\text { 4-allyl resorcinol, } \\
\text { chavibetol }\end{array}$ & $\begin{array}{c}2 \mathrm{Mpa}, \\
10-90 \mathrm{~min}, \\
50-250{ }^{\circ} \mathrm{C}, \\
0.25-1 \mathrm{~mm}, \\
1-4 \mathrm{~mL} / \mathrm{min}\end{array}$ & HPLC-UV & $\begin{array}{c}\text { convention (water } \\
\text { 92.2-111\%; } \\
\text { methanol } \\
96.6-110 \%)\end{array}$ & [177] \\
\hline $\begin{array}{l}\text { Aquilaria } \\
\text { malaccensis }\end{array}$ & leaves & $\begin{array}{c}\text { nonacosane, } \\
\text { triacontane, } \\
\text { pentadecanal, } \\
\text { 9-octadecenal, }(\mathrm{Z})-\text {-, } \\
\text { tetradecanal, } \\
\text { tetrapentacontane, } \\
\text { guaiacol }\end{array}$ & $\begin{array}{l}100-271^{\circ} \mathrm{C}, \\
1-34 \mathrm{~min}\end{array}$ & $\begin{array}{c}\text { GC/MS, SEM, } \\
\text { BET }\end{array}$ & & [178] \\
\hline
\end{tabular}


Table 6. Cont

\begin{tabular}{|c|c|c|c|c|c|c|}
\hline Samples & $\begin{array}{l}\text { Medicinal } \\
\text { Parts }\end{array}$ & $\begin{array}{l}\text { Compounds } \\
\text { Extracted }\end{array}$ & $\begin{array}{l}\text { Extraction } \\
\text { Conditions }\end{array}$ & Methods & $\begin{array}{l}\text { Other Extraction } \\
\text { Methods (Solvent, } \\
\text { Ratios of Yields) }\end{array}$ & Ref. \\
\hline laurel & leaves & $\begin{array}{c}\alpha \text {-phellandrene, } \\
\beta \text {-pinene, 1,8-cineole, } \\
\text { borneol, } \\
\text { nona-3,7-dienol, } \\
\text { isobornyl acetate, } \\
\gamma \text {-terpineol, etc. }\end{array}$ & $\begin{array}{c}15 \mathrm{~min}, \\
50-200^{\circ} \mathrm{C}, \\
1.5-15 \mathrm{Mpa}, \\
0.5-5.0 \mathrm{~mL} / \mathrm{min}\end{array}$ & $\begin{array}{l}\text { GC-MS, } \\
\text { GC-FID }\end{array}$ & & [179] \\
\hline Citrus hystrix & leaves & $\begin{array}{l}\text { linalool, isopulegol, } \\
\text { neoisopulegol, } \\
\text { citronellal, } \\
\text { 4-terpineol, } \\
\text { citronellol, geraniol, } \\
\text { menthoglycol, etc. }\end{array}$ & $\begin{array}{l}120-180{ }^{\circ} \mathrm{C} \\
5-20 \mathrm{~g} / \mathrm{mL} \\
5-30 \mathrm{~min}\end{array}$ & GC-MS & $\operatorname{HD}(28.2 \%)$ & [180] \\
\hline $\begin{array}{l}\text { Coriandrum } \\
\text { sativum L. }\end{array}$ & $\begin{array}{l}\text { coriander } \\
\text { seeds }\end{array}$ & $\begin{array}{c}\alpha \text {-pinene, } \beta \text {-pinene, } \\
\text { camphor, } \\
\text { methylchavicol, } \\
\gamma \text {-terpinene, linalool, } \\
\text { geraniol, carvacrol, } \\
\text { etc. }\end{array}$ & $\begin{array}{c}100-200{ }^{\circ} \mathrm{C} \\
1: 10 \mathrm{~g} / \mathrm{mL} \\
2 \mathrm{Mpa}, 20 \mathrm{~min}\end{array}$ & $\begin{array}{l}\text { GC-MS, } \\
\text { GC-FID }\end{array}$ & $\begin{array}{c}\mathrm{HD}(27.0 \%) \\
\text { Soxhlet }(\mathrm{DCM} \\
6.5 \text {-fold), } \mathrm{SCCO}_{2} \\
\text { (4-fold) }\end{array}$ & [181] \\
\hline Lavandula L. & $\begin{array}{l}\text { lavender } \\
\text { flowers }\end{array}$ & $\begin{array}{l}\text { a-thujene, a-pinene, } \\
\text { camphene, sabinene, } \\
\text { pinene, myrcene, } \\
\text { hexylacetate, } \\
\text { terpinene, limonene, } \\
\text { etc. }\end{array}$ & $\begin{array}{c}125^{\circ} \mathrm{C}, 3 \mathrm{Mpa}, \\
30 \mathrm{~min}\end{array}$ & GC-MS, FID & $\begin{array}{c}\text { HD (1.2-fold), } \\
\text { US-HD (1.3-fold), } \\
\text { NaCl-HD (1.3-fold) }\end{array}$ & [182] \\
\hline \multicolumn{7}{|c|}{ Alkaloids } \\
\hline $\begin{array}{c}\text { Sophora } \\
\text { flavescens Ait. }\end{array}$ & root & $\begin{array}{l}\text { cytisine, matrine, } \\
\text { sophoridine, } \\
\text { sophocarpine, } \\
\text { oxymatrine }\end{array}$ & $\begin{array}{c}70-190{ }^{\circ} \mathrm{C}, \\
5-14 \mathrm{~min}, \\
4.0-13.8 \mathrm{Mpa}\end{array}$ & CE & $\begin{array}{c}\text { ASE (ethanol } \\
78.1 \% \text { ) }\end{array}$ & [16] \\
\hline black tea brick & leaves & $\begin{array}{l}\text { theophylline, } \\
\text { epicatechin gallate, } \\
\text { caffeine, etc. }\end{array}$ & $\begin{array}{c}120-180{ }^{\circ} \mathrm{C}, \\
7-42 \mathrm{~min}, \\
6-18 \mathrm{~mL} / \mathrm{min}\end{array}$ & HPLC & & [46] \\
\hline $\begin{array}{l}\text { Symphytum } \\
\text { officinale L. }\end{array}$ & root & $\begin{array}{l}\text { lycopsamine, } \\
\text { echimidine, } \\
\text { lasiocarpine, } \\
\text { symviridine }\end{array}$ & $\begin{array}{l}60-120^{\circ} \mathrm{C} \\
40 \mathrm{~min}\end{array}$ & $\begin{array}{c}\text { HPLC, LC-MS, } \\
\mathrm{MS}^{\mathrm{n}}\end{array}$ & $\begin{array}{l}\text { HRE (methanol } \\
\text { 2.8-fold) }\end{array}$ & [183] \\
\hline $\begin{array}{l}\text { hydrastis } \\
\text { canadensis }\end{array}$ & root & hydrastine, berberine & $\begin{array}{c}100-160{ }^{\circ} \mathrm{C}, 1- \\
10 \mathrm{Mpa}, \\
5-60 \mathrm{~min}, \\
0.5-1.5 \mathrm{~mL} / \mathrm{min}\end{array}$ & HPLC-DAD & $\begin{array}{l}\text { reflux (methanol } \\
90.8 \% \text { ), UAE } \\
\text { (methanol 106\%) }\end{array}$ & [184] \\
\hline cocoa & shells & $\begin{array}{l}\mathrm{TP} \text {, theobromine, } \\
\text { theophylline, } \\
\text { caffeine, epicatechin, } \\
\text { etc. }\end{array}$ & $\begin{array}{c}120-220{ }^{\circ} \mathrm{C}, \\
15-75 \mathrm{~min}, \\
1: 10-1: 30 \mathrm{~g} / \mathrm{mL}\end{array}$ & HPLC, UV & & [185] \\
\hline $\begin{array}{c}\text { Musaceae, Beta } \\
\text { vulgaris }\end{array}$ & peels & $\begin{array}{l}\text { dopamine, total } \\
\text { betacyanin, } \\
\text { betaxanthin }\end{array}$ & $\begin{array}{c}150{ }^{\circ} \mathrm{C}, 5 \mathrm{~min} \\
3 \mathrm{Mpa} \\
1: 20 \mathrm{~g} / \mathrm{mL}\end{array}$ & HPLC, UV-Vis & $\begin{array}{l}\text { infusion }(100 \%), \\
\text { decoction }(1.2 \text {-fold), } \\
\text { maceration }(97.4 \%) \text {, } \\
\text { UAE }(101 \%), \text { MAE } \\
(50.3 \%)\end{array}$ & [186] \\
\hline
\end{tabular}


Table 6. Cont.

\begin{tabular}{|c|c|c|c|c|c|c|}
\hline Samples & $\begin{array}{l}\text { Medicinal } \\
\text { Parts }\end{array}$ & Compounds Extracted & $\begin{array}{l}\text { Extraction } \\
\text { Conditions }\end{array}$ & Methods & $\begin{array}{l}\text { Other Extraction } \\
\text { Methods (Solvent, } \\
\text { Ratios of Yields) }\end{array}$ & Ref. \\
\hline $\begin{array}{l}\text { Rheum } \\
\text { tanguticum }\end{array}$ & root & damnacanthal & $\begin{array}{c}33-67 \mathrm{~min}, \\
100-200{ }^{\circ} \mathrm{C}, 1.4- \\
4.6 \mathrm{~mL} / \mathrm{min}\end{array}$ & $\begin{array}{l}\text { HPLC, NMR, } \\
\text { HSCCC }\end{array}$ & & {$[17]$} \\
\hline $\begin{array}{l}\text { Garcinia } \\
\text { mangostana } \\
\text { Linn }\end{array}$ & $\begin{array}{l}\text { mangosteen } \\
\text { pericarps }\end{array}$ & $\mathrm{TP}$, xanthone & $\begin{array}{l}120-160{ }^{\circ} \mathrm{C}, \\
1-10 \mathrm{Mpa}, \\
5-60 \mathrm{~min}, \\
10-30 \% \text { DES }\end{array}$ & $\begin{array}{l}\text { UV-vis, FT-IR, } \\
\text { SEM }\end{array}$ & & [188] \\
\hline $\begin{array}{l}\text { Phaleria } \\
\text { macrocarpa }\end{array}$ & $\begin{array}{c}\text { mahkota } \\
\text { dewa fruits }\end{array}$ & mangiferin & $\begin{array}{l}4.0 \mathrm{Mpa}, 5 \mathrm{~h}, \\
50-150{ }^{\circ} \mathrm{C}\end{array}$ & HPLC & & [189] \\
\hline $\begin{array}{l}\text { Lithospermum } \\
\text { erythrorhizon }\end{array}$ & root & $\begin{array}{c}\text { shikonin, } \\
\text { acetylshikonin, } \beta \text { - } \\
\text { dimethylacrylshikonin, } \\
\text { etc. }\end{array}$ & $\begin{array}{c}40-60 \text { mesh, } \\
120{ }^{\circ} \mathrm{C}, 5 \mathrm{Mpa}\end{array}$ & $\begin{array}{c}\text { UV, } \\
\text { HPLC-ELSD }\end{array}$ & $\begin{array}{l}\mathrm{SCCO}_{2}(86.3 \%), \\
\text { Soxhlet (ethyl } \\
\text { acetate } 95.4 \%), \\
\text { UWE (1.4-fold) }\end{array}$ & [190] \\
\hline $\begin{array}{l}\text { Morinda } \\
\text { citrifolia }\end{array}$ & root & alizarin & $\begin{array}{c}4 \mathrm{Mpa}, 150 \text { and } \\
220{ }^{\circ} \mathrm{C} \\
1.6-4 \mathrm{~mL} / \mathrm{min}\end{array}$ & RP-HPLC-UV & & [191] \\
\hline $\begin{array}{l}\text { Morinda } \\
\text { citrifolia }\end{array}$ & root & $\begin{array}{c}1,2- \\
\text { dihydroxyanthraquinone, } \\
\text { alizarin }\end{array}$ & $\begin{array}{r}110-220{ }^{\circ} \mathrm{C} \\
2-6 \mathrm{~mL} / \mathrm{min}\end{array}$ & UV-Vis & ethanol (3d) & [192] \\
\hline $\begin{array}{l}\text { Morinda } \\
\text { citrifolia }\end{array}$ & root & & $\begin{array}{c}4 \mathrm{Mpa}, \\
150-200{ }^{\circ} \mathrm{C}, \\
2-6 \mathrm{~mL} / \mathrm{min}\end{array}$ & UV-Vis & $\begin{array}{c}\text { convention } \\
\text { (ethanol 81.16\%), } \\
\text { Soxhlet (ethanol } \\
\text { 97.94\%), UAE } \\
\text { (ethanol } 79.62 \% \text { ) } \\
\text { SWBE }(96.41 \% \text { ) }\end{array}$ & [193] \\
\hline \multicolumn{7}{|c|}{ Terpenes } \\
\hline $\begin{array}{l}\text { Hedyotis diffusa } \\
\text { Willd. }\end{array}$ & $\begin{array}{l}\text { whole } \\
\text { plants }\end{array}$ & ursolic acid & $\begin{array}{c}120-200{ }^{\circ} \mathrm{C}, \\
10-50 \mathrm{~min}, \\
20-40 \mathrm{~mL} / \mathrm{g} \\
0.6-3.0 \mathrm{Mpa}\end{array}$ & $\begin{array}{c}\text { HPLC-ESI-TOF- } \\
\text { MS }\end{array}$ & $\begin{array}{c}\text { maceration } \\
\text { (ethanol } 58.8 \% \text { ), } \\
\text { HRE (ethanol } \\
78.4 \% \text { ), UAE } \\
\text { (ethanol } 90.4 \% \text { ), } \\
\text { MAE (ethanol } \\
74.9 \% \text { ) }\end{array}$ & [13] \\
\hline Centella asiatica & $\begin{array}{l}\text { whole } \\
\text { plants }\end{array}$ & $\begin{array}{l}\text { asiatic acid, } \\
\text { asiaticoside }\end{array}$ & $\begin{array}{c}100-250{ }^{\circ} \mathrm{C}, \\
10-40 \mathrm{Mpa}, 5 \mathrm{~h}\end{array}$ & HPLC, DLS & & {$[14]$} \\
\hline basil, oregano & leaves & $\begin{array}{l}\text { limonene, citronellol, } \\
\text { etc. }\end{array}$ & $\begin{array}{l}100 \text { and } 150{ }^{\circ} \mathrm{C}, \\
10 \mathrm{~min}\end{array}$ & GC-FID & & {$[48]$} \\
\hline $\begin{array}{l}\text { Ganoderma } \\
\text { lucidum }\end{array}$ & fruits & $\begin{array}{c}\text { ganodermanon-triol, } \\
\text { ganoderic acids, } \\
\text { lucidumol }\end{array}$ & $\begin{array}{l}100-200{ }^{\circ} \mathrm{C} \\
5-10 \mathrm{Mpa} \\
5-60 \mathrm{~min}\end{array}$ & $\begin{array}{l}\text { HPLC, SEC-UV, } \\
\text { SEM, } \\
\text { MALDI-TOF }\end{array}$ & & [194] \\
\hline $\begin{array}{l}\text { Orostachys } \\
\text { japonicus }\end{array}$ & $\begin{array}{l}\text { stems, } \\
\text { leaves }\end{array}$ & $\begin{array}{c}\text { triterpene, camellia, } \\
\text { etc. }\end{array}$ & $\begin{array}{l}110-260{ }^{\circ} \mathrm{C} \\
5-20 \mathrm{~min} \\
10 \mathrm{Mpa}\end{array}$ & HPLC-MS & & [195] \\
\hline Betula pendula & birch bark & betulinic acid & $\begin{array}{l}160-200{ }^{\circ} \mathrm{C}, \\
10-30 \mathrm{~min}, \\
10 \mathrm{Mpa}\end{array}$ & HPLC & & [196] \\
\hline Inula racemose & plants & $\begin{array}{l}\text { igalan, soalantolactone, } \\
\text { alantolactone }\end{array}$ & $\begin{array}{c}23.2-56.8 \mathrm{~min}, \\
1.3-4.7 \\
\mathrm{~mL} / \mathrm{min}, \\
129.5-230.5^{\circ} \mathrm{C}\end{array}$ & $\begin{array}{c}\text { HPLC, } \\
{ }^{1} \mathrm{H}-\mathrm{NMR} \\
{ }^{13} \mathrm{C}-\mathrm{NMR}, \mathrm{MS}\end{array}$ & $\begin{array}{l}\text { Soxhlet (ethanol } \\
100 \%), \text { UAE } \\
\text { (ethanol 70.36\%), } \\
\mathrm{SCCO}_{2}(76.06 \%)\end{array}$ & [197] \\
\hline
\end{tabular}


Table 6. Cont.

\begin{tabular}{|c|c|c|c|c|c|c|}
\hline Samples & $\begin{array}{l}\text { Medicinal } \\
\text { Parts }\end{array}$ & Compounds Extracted & $\begin{array}{l}\text { Extraction } \\
\text { Conditions }\end{array}$ & Methods & $\begin{array}{l}\text { Other Extraction } \\
\text { Methods (Solvent, } \\
\text { Ratios of Yields) }\end{array}$ & Ref. \\
\hline $\begin{array}{l}\text { Semen } \\
\text { richonsanthis }\end{array}$ & seeds & $\begin{array}{c}\text { 3,29- } \\
\text { dibenzoylkarounidiol, } \\
\text { polysaccharides }\end{array}$ & $\begin{array}{c}80-160{ }^{\circ} \mathrm{C}, \\
5.0-30.0 \mathrm{~min}\end{array}$ & $\begin{array}{l}\text { HPLC, UV, } \\
\text { SEM }\end{array}$ & & [198] \\
\hline Cucurbita pepo & $\begin{array}{l}\text { pumpkin } \\
\text { peel }\end{array}$ & $\begin{array}{l}14 \text { carotenoid } \\
\text { compounds }\end{array}$ & $\begin{array}{c}120^{\circ} \mathrm{C}, 3 \mathrm{~h}, \\
5 \mathrm{Mpa}\end{array}$ & UV, HPLC & $\mathrm{SCCO}_{2}(75.4 \%)$ & [199] \\
\hline Betula pendula & birch bark & $\begin{array}{l}\text { sesquiterpenes, } \\
\text { steroids }\end{array}$ & $\begin{array}{c}10 \mathrm{~min}, \\
100-200{ }^{\circ} \mathrm{C}\end{array}$ & $\begin{array}{l}\text { LC, GC/MS, } \\
\text { NMR }\end{array}$ & & [200] \\
\hline S. rebaudiana & $\begin{array}{l}\text { Bertoni } \\
\text { leaves }\end{array}$ & $\begin{array}{l}\text { steviol glycosides, } \\
\text { tannins, chlorophyll A }\end{array}$ & $\begin{array}{c}100-160{ }^{\circ} \mathrm{C}, \\
5-10 \mathrm{~min} \\
10.34 \mathrm{Mpa}, \\
1: 3 \mathrm{~g} / \mathrm{mL}\end{array}$ & $\begin{array}{l}\text { HPLC, UV, } \\
\text { UV/Vis }\end{array}$ & & [201] \\
\hline \multicolumn{7}{|c|}{ Lignans } \\
\hline $\begin{array}{l}\text { Linum } \\
\text { usitatissimum L. }\end{array}$ & flaxseed & $\begin{array}{l}\text { SDG lignan, pHenolics, } \\
\text { flavonoids }\end{array}$ & $\begin{array}{l}160-180{ }^{\circ} \mathrm{C}, \\
5-60 \mathrm{~min} \\
10 \mathrm{Mpa}\end{array}$ & $\begin{array}{c}\text { HPLC-MS/MS, } \\
\text { UV }\end{array}$ & & [41] \\
\hline $\begin{array}{l}\text { Sesamum } \\
\text { indicum L. }\end{array}$ & $\begin{array}{l}\text { sesame } \\
\text { seeds }\end{array}$ & $\begin{array}{l}\text { lignans, } \mathrm{TP}, \text { flavonoids, } \\
\text { flavonols }\end{array}$ & $\begin{array}{c}140-220{ }^{\circ} \mathrm{C} \\
8-14 \mathrm{Mpa} \\
0-95 \% \text { ethanol, } \\
0-75 \mathrm{~min}\end{array}$ & UV & & [42] \\
\hline $\begin{array}{l}\text { Linum } \\
\text { usitatissimum L. }\end{array}$ & flaxseed & $\begin{array}{c}\text { total fat content, SDG } \\
\text { lignan }\end{array}$ & $\begin{array}{c}120-180{ }^{\circ} \mathrm{C}, \\
15-90 \mathrm{~min}, \\
10-13.8 \mathrm{Mpa}\end{array}$ & $\begin{array}{c}\text { HPLC-MS/MS, } \\
\text { UV }\end{array}$ & & [43] \\
\hline $\begin{array}{l}\text { Sinopodophyllum } \\
\text { hexandrum }\end{array}$ & root & podophyllotoxin & $\begin{array}{c}12 \mathrm{~mL} / \mathrm{g} \\
3 \mathrm{Mpa} \\
2 \mathrm{ml} / \mathrm{min}, \\
120-240^{\circ} \mathrm{C}\end{array}$ & HPLC & & [202] \\
\hline \multicolumn{7}{|c|}{ Steroids } \\
\hline $\begin{array}{l}\text { Pfaffia glomerata, } \\
\text { Amaranthaceae }\end{array}$ & ginseng root & $\begin{array}{c}\text { sugar, } \\
\text { fructooligosaccharides, } \\
\text { beta-ecdysone }\end{array}$ & $\begin{array}{l}80-180{ }^{\circ} \mathrm{C} \\
5-15 \mathrm{~min} \\
2-12 \mathrm{Mpa}\end{array}$ & $\begin{array}{l}\text { HPLC-ELSD, } \\
\text { HPLC }\end{array}$ & & [24] \\
\hline $\begin{array}{l}\text { Panax ginseng } \\
\text { C.A. Meyer }\end{array}$ & ginseng root & $\begin{array}{l}\mathrm{TP}, \text { maltol, panaxadiol, } \\
\text { panaxatriol }\end{array}$ & $\begin{array}{l}150-200{ }^{\circ} \mathrm{C}, \\
5-30 \mathrm{~min} \\
100 \mathrm{Mpa}\end{array}$ & HPLC, UV & $\begin{array}{c}\text { convention (water } \\
32.6 \% \text {; methanol } \\
24.1 \% \text {; ethanol } \\
18.7 \% \text { ) }\end{array}$ & [25] \\
\hline $\begin{array}{l}\text { Panax ginseng } \\
\text { C.A. Meyer }\end{array}$ & ginseng root & $\begin{array}{l}\text { total ginsenosides, total } \\
\text { sugar, } \\
\text { 1-oleanane } \\
\text { ginsenosides, etc. }\end{array}$ & $\begin{array}{c}120-200{ }^{\circ} \mathrm{C}, \\
20 \mathrm{~min} \\
1: 20 \mathrm{~g} / \mathrm{mL} \\
6.0 \mathrm{Mpa}\end{array}$ & $\begin{array}{c}\text { FT-IR, UV, } \\
\text { UFLC-MS/MS }\end{array}$ & $\begin{array}{l}\text { heating (water, } \\
30.9 \% \text {; ethanol } \\
94.4 \% \text { ) }\end{array}$ & [26] \\
\hline grapevine & $\begin{array}{c}\text { root, } \\
\text { wood, cane }\end{array}$ & $\begin{array}{l}\text { E-piceid, E-piceatannol, } \\
\text { E-resveratrol, } \\
\text { E-parthenocissin, etc. }\end{array}$ & $\begin{array}{c}100-190{ }^{\circ} \mathrm{C} \\
\text { 5-30 min, } \\
10 \mathrm{Mpa}\end{array}$ & $\begin{array}{c}\text { LC-DAD/ESI- } \\
\text { IT, Q-TOF, } \\
\text { NMR }\end{array}$ & $\begin{array}{c}\text { ASE (116\% for cane; } \\
103 \% \text { for wood; } \\
1.5 \text {-fold for root) }\end{array}$ & [203] \\
\hline
\end{tabular}

\subsection{Alkaloids}

Alkaloids are a class of basic nitrogen-containing organic compounds with a great structure diversity, most of which are heterocyclic compounds, and the nitrogen atom is in the heterocyclic ring. Most alkaloids are alkaline and have therapeutic and recreational activities. Alkaloid-containing plants have been used in folk medicine for centuries. Therefore, many researches are paid attention to the extraction and separation of alkaloids from plants. Traditional extractions include organic solvents, such as methanol $[183,184]$, 
ethanol [183], or an acidic solution [187]. Recently, SBWE was successfully applied to the extraction or separation of alkaloids from plants or animals, as illustrated in Table 6.

Due to the diversity and relatively poor thermal stability of alkaloids, the optimal extraction methods depend on the pHysio-chemical characteristics of alkaloids. Liu et al. [16] used SBWE and capillary electrophoresis (CE) to extract and determine cytisine, sophocarpine, matrine, sophoridine, and oxymatrine in Sophora flavescens Ait., which is a traditional Chinese medicine. The extraction yields obtained using SBWE, ASE, UAE, and chloroform soaking extraction methods were co Mpared. SBWE needs a short extraction time, as there is no need for organic solvent consumption and it exhibited the highest extraction efficiency for the total alkaloid yield. Similarly, Torto et al. [184] concluded that both SBWE and conventional methods achieved co Mparable extraction yields, while reflux and UAE were slower (over $6 \mathrm{~h}$ ) and employed large quantities of organic solvents. Therefore, the SBWE method was simple and relatively fast for extraction. However, Liu et al. [183] showed that LC-ESI-obitrap MS provides a powerful method for the identification and determination of hepatotoxic pyrrolizidine alkaloids, and reflux showed a higher extraction efficiency co Mpared with SBWE. Komes and his collaborators [186] investigated conventional and innovative extraction techniques (SBWE, UAE). They found both extracts of banana and beetroot peels obtained by THD $\left(100{ }^{\circ} \mathrm{C}, 20 \mathrm{~min}\right)$ exhibited the highest total pHenolic content and antioxidant capacity. Extraction by infusion $\left(80^{\circ} \mathrm{C}\right.$, $30 \mathrm{~min}$ ) yielded a beetroot peel extract with the highest total betacyanin content. The optimal extraction temperature and time for alkaloids is also listed in Figure 9.

\subsection{Quinones}

The quinones are a kind of organic components that have quinone structures, which can mainly be divided into four types: benzoquinone, naphthoquinone, pHenanthraquinone, and anthraquinone. Anthraquinone and its derivatives widely exist in plants and can be obtained from many plants, especially conifers. Some quinones have desirable pHarmacological properties, such as purgative, antimicrobial and antiparasitic, anti-cardiovascular roles, etc. Extraction of quinones from natural products has utilized ethyl acetate [190], SFE [190], ethanol [191,193], or water as extraction solvents, shown in Table 6.

Machmudah et al. [188] performed extraction of pHenolic compounds from pericarps of mangosteen by subcritical water treatment at temperatures and pressures of 120 to $160{ }^{\circ} \mathrm{C}$ and 1 to $10 \mathrm{Mpa}$ in batch and semi-batch systems. They added 10 to $30 \%$ deep eutectic solvent (DES) to subcritical water, and the results showed that with $30 \%$ DES, the yields of xanthone and pHenolic compounds content were $24.87 \mathrm{mg} / \mathrm{g}$ dried sample and $179.54 \mathrm{mg}$ of gallic acid equivalent/g dried sample at extraction temperatures of 160 and $120^{\circ} \mathrm{C}$ in the batch system, respectively. The addition of DES in SBWE process could accelerate hydrolysis reaction to extract plant biomass components matrix.

Morinda citrifolia (Noni), planted in tropical Asia, has been used in folk remedies to treat various kinds of diseases and symptoms. Shotipruk and coworkers have conducted a series of experiments on this plant [191-193]. They used a continuous flow system to extract damnacanthal, alizarin, and 1,2-dihydroxyanthraquinone. They found that pressure had no significant effect on the results for the range $110-220^{\circ} \mathrm{C}$. Co Mpared with conventional extraction, SBWE and Soxhlet resulted in extracts that have the highest antioxidant activity. The data were fitted with mathematic models to determine the extraction mechanism. The results suggested that the overall extraction mechanism was influenced by solute partitioning equilibrium with external mass transfer through liquid film.

The optimal extraction temperature and time for quinones in SBWE are shown in Figure 10. The optimal extraction temperature of quinones is between 160 to $170{ }^{\circ} \mathrm{C}$ and the time is between about 60 to $120 \mathrm{~min}$. 


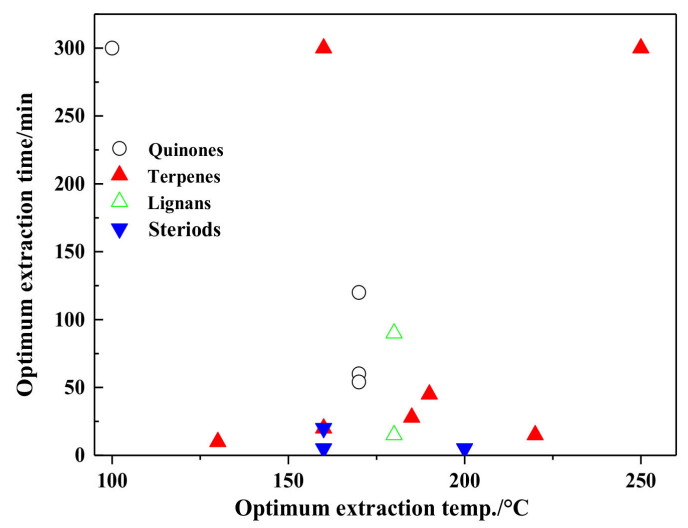

Figure 10. Optimum extraction conditions for quinones, terpenes, lignans, and steroids.

\subsection{Terpenes}

Terpenes are a large and diverse class of organic compounds using isoprene as the basic structure unit, which widely exists in plants and some insects, and can be obtained from many plants, especially conifers. Terpenes have wide varieties and may be classified by the number of isoprene units in the molecule, such as monoterpenes, hemiterpenes, sesquiterpenes, diterpenes, triterpenes, tetraterpenes, and polyterpenes. Terpenes are of importance for the use in food, cosmetics, and pHarmaceutical industries. However, the extraction of terpenes and terpenoids from natural products is often problematic, illustrated in Table 6 . Consequently, they are mainly produced by industrial synthesis, usually from petrochemicals.

Chen et al. [197] employed SBWE for the efficient extraction sesquiterpene lactones from I. racemose. Extraction time (23.2-56.8 $\mathrm{min})$, temperature $\left(129.5-230.5{ }^{\circ} \mathrm{C}\right)$ and flow rate $(1.3-4.7 \mathrm{~mL} / \mathrm{min})$ were investigated. A co Mparison of SBWE with traditional extraction technologies (Soxhlet, UAE, and SFE) showed that subcritical water could be a green and efficient substitution for the extraction of sesquiterpene lactones from I. racemose. Xiao et al. [13] developed SBWE for extraction of ursolic acid from Hedyotis diffusa. The RSM model proved to predict the experimental results very well and demonstrated that UA yield was mainly dependent on solvent/solid ratio, followed by particle size and temperature. Four extraction methods (UAE and SBWE) were co Mparatively analyzed, which indicated that SBWE was a time-saving, cost-saving, and environment-friendly extraction technology for the extraction of UA from Hedyotis fusa. Other researchers achieved the same experimental results, except for Falev et al. [200], who found that subcritical water is a poor solvent for pentacyclic triterpenes. Extraction with subcritical solvents (aliphatic alcohols, acetonitrile, and chloroform) is the most rapid and efficient way to isolate pentacyclic triterpenes from plant raw materials.

The optimal extraction temperature and time for terpenes in SBWE are summarized in Figure 10. The optimal extraction temperature of quinones is between 130 and $225^{\circ} \mathrm{C}$, and the time is between about 10 and $50 \mathrm{~min}$.

\subsection{Lignans}

Lignans are a kind of natural compound formed by the polymerization of two molecules of pHenylpropanine derivatives (i.e., $\mathrm{C}_{3}-\mathrm{C}_{6}$ monomers), which exist in plants and belong to pHytoestrogens. The monomers composing of lignans include cinnamic acid, cinnamyl alcohol, acryl benzene, allylbenzene, and so on. Lignans are reported to have potential antitumor, anti-inflammatory, or antioxidant activity in the laboratory models of human diseases. Most lignans are lipophilic, and easily soluble in organic solvents. Therefore, extraction of lignans from plants often utilized ethanol, ether, or acetone as extraction solvents. Researches about SBWE of lignans are only a few papers, as listed in Table 6. 
Evrim $[48,50]$ has conducted a detailed investigation of the material shape (flaxseed, ground flaxseed meal, and flaxseed meal sticks), temperature, extraction time, pressure, fresh water, and sample amount on the effect of secoisolariciresinol diglucoside lignan content using accelerated solvent extractor. The highest amount $(12.94 \mathrm{mg} / \mathrm{g})$ and extraction yield $\left(72.57 \%\right.$ ) were obtained at $180^{\circ} \mathrm{C}$ for $15 \mathrm{~min}, 10.3 \mathrm{Mpa}$, and $40 \%$ fresh water using $5 \mathrm{~g}$ of flaxseed meal sticks. Bodoira et al. [42] used water and ethanol under sub-critical conditions to extracted bioactive compounds from sesame (Sesamum indicum L.) defatted seeds. At $220^{\circ} \mathrm{C}, 8 \mathrm{Mpa}$ and $63.5 \%$ ethanol as co-solvent condition, the yields of lignans, $\mathrm{TP}$, flavonoids compounds were maximized, and the antioxidants were similar to those reached by using synthetic antioxidants. Kinetic studies showed a high extraction rate of pHenolic compounds until the first 50 min of extraction, and it was in parallel with the highest scavenging capacity. SBWE could selectively extract different kinds of bioactive compounds only by changing process conditions. The optimal extraction temperature for lignans is about $180^{\circ} \mathrm{C}$, as shown in Figure 10.

\subsection{Steroids}

A steroid is a class of natural chemical components widely existing in nature, including pHytosterin, bile acids, $\mathrm{C}_{21}$ steroids, insect allergic hormones, cardiac glycosides, steroidal saponins, steroidal alkaloids, bufogenin, etc. The steroid core structure has the basic skeleton structure of cyclopentano-perhydrophenthrene, bonded in four "fused" rings: three six-member cyclohexane rings and one five-member cyclopentane ring. Steroid compounds have various biological activities. They have a wide range of applications. The extraction methods of steroids from natural products include maceration, Soxhlet, and SBWE are illustrated in Table 6.

Ginseng is a well-known traditional Chinese medicine with numerous pHarmacological effects [24-26]. These bioactive components are mainly the ginsenosides, polyphenols, amino acids, and polysaccharides. Renata et al. [24] utilized SBWE to obtain fructooligosaccharides and beta-ecdysone from Brazilian ginseng root and aerial parts. Lee et al. [25] extracted red ginseng by varying the temperature $\left(150-200^{\circ} \mathrm{C}\right)$ and extraction times (5-30 min) in SBWE. Co Mpared to traditional heat extraction methods (ethanol, hot water, and methanol), extracts of red ginseng from SBWE had higher ginsenoside concentrations and antioxidative properties. Shivraj et al. [204] applied subcritical water to extract withanosides and withanolides from ashwagandha at varying temperatures (100$200{ }^{\circ} \mathrm{C}$ ) and extraction times (10-30 min). Various biological effects, including cytotoxicity, antioxidant, and enzyme inhibitory activities were quantified using HPLC. Withaferin A showed significant reduction in cell viability of cervical cancer cells, with $\mathrm{IC}_{50}$ values $10 \mathrm{mg} / \mathrm{mL}$ and $8.5 \mu \mathrm{M} / \mathrm{mL}$, respectively, but no cytotoxic effect for normal cells. Thus, SBWE could be used for extraction of pHarmacologically active fractions with therapeutic applications. The optimal extraction temperature for steroids is about $160^{\circ} \mathrm{C}$, as shown in Figure 10.

\section{Conclusions and Future Perspectives}

Due to its nontoxic, nonflammable, and widely available nature, SBWE of natural products has gained greater attention during the last decade. Our review of over 200 articles shows that SBWE is a promising technology in extracting natural products. A wide variety of plant-related materials have been extracted by subcritical water. Sample materials include medicinal herbs, seasoning herbs, vegetables, fruits, algae, shrubs, tea leaves, grains, and seeds. The following natural products have been extracted by SBWE: Alkaloids, carbohydrates, essential oil, flavonoids, glycosides, lignans, organic acids, polyphenolics, quinones, steroids, and terpenes. Both static and dynamic mode are employed in SBWE. In general, the static extraction efficiency is lower than that of the dynamic mode. Thus, most SBWE experiments were conducted using the static mode, and then, followed by the dynamic mode. SBWE system was also coupled with HPLC, subcritical water chromatography, and high-temperature liquid chromatography. Co-solvents such as ethanol, 
methanol, salts, and ionic liquids were also used to improve SBWE efficiency. It should be noted that temperature has the most significant effect on SBWE efficiency, and thus, it can be optimized. The optimal temperature ranges from 120 to $200{ }^{\circ} \mathrm{C}$ for extracting the natural products mentioned above.

A major advantage of SBWE of natural products is that water is nontoxic, and therefore it is more suitable for the extraction of herbs, vegetables, and fruits since the extracts can be safely consumed by human or animals. In addition, if no organic modifiers are used in SBWE, the liquid waste generated after SBWE does not require waste disposal. However, the high temperature used in SBWE may potentially cause analyte degradation. Thus, one must evaluate analyte stability under the temperature conditions to be used in SBWE to ensure that the analytes extracted do not undergo degradation during SBWE. SBWE employs high-pressure and high-temperature fluid, and great caution is required to ensure safe operation of the SBWE system. In addition, frequent pluming blockage may occur during dynamic SBWE process.

A vast majority of SBWE research reported is studied at the bench scale. The next level of SBWE should be scaling up to the industrial level. Results of some pilot scale studies have demonstrated the potential development of large scale SBWE processes.

Author Contributions: Y.C. and Y.Y. designed the project; Y.C. drafted and edited the manuscript; Y.Y. helped draft the manuscript, provided guidance, rewrote sections, edited each draft of the manuscript; S.Y., F.X., S.D. carried out literature search and helped in writing manuscript. All authors have read and agreed to the published version of the manuscript.

Funding: The review was supported by the China Scholarship Council (201909370008), and by the Natural Science Foundation of Shandong Province (grant references: ZR2020QB177), and Shandong Key Research \& Development Plan (2020CXGC010506), and Demonstration Project of Integration of Science, Education and Industry in Qilu University of Technology (2020KJC-ZD09).

Institutional Review Board Statement: Not applicable.

Informed Consent Statement: Not applicable.

Data Availability Statement: Data sharing not applicable. No new data were created or analyzed in this study.

Acknowledgments: The authors extend their appreciation to the China Scholarship Council and East Carolina University.

Conflicts of Interest: The authors declare no conflict of interest.

\section{References}

1. Colegate, S.M.; Molyneux, R.J. Bioactive Natural Products; CRC Press: Boca Raton, FL, USA, 1993.

2. Fernández, D.P.; Goodwin, A.R.H.; Lemmon, E.W.; Sengers, J.M.H.L.; Williams, R.C. A formulation for the static permittivity of water and steam at temperatures from $238 \mathrm{~K}$ to $873 \mathrm{~K}$ at pressures up to $1200 \mathrm{Mpa}$, including derivatives and Debye-Hückel coefficients. J. pHys. Chem. Ref. Data 1997, 26, 1125-1166. [CrossRef]

3. Yang, Y. Subcritical water chromatography: A green approach to high-temperature liquid chromatography. J. Sep. Sci. 2007, 30, 1131-1140. [CrossRef]

4. Zhang, J.; Wen, C.; Zhang, H.; Duan, Y.; Ma, H. Recent advances in the extraction of bioactive compounds with subcritical water: A review. Trends Food Sci. Technol. 2020, 95, 183-195. [CrossRef]

5. Yang, Y.; Hawthorne, S.B.; Miller, D.J. Class-selective extraction of polar, moderately polar, and nonpolar organics from hydrocarbon wastes using subcritical water. Environ. Sci. Technol. 1997, 31, 430-437. [CrossRef]

6. Sushkova, S.N.; Vasilyeva, G.K.; minkina, T.M.; Mandzhieva, S.S.; Tjurina, I.G.; Kolesnikov, S.I.; Kizilkaya, R.; Askin, T. New method for benzo [a]pyrene analysis in plant material using subcritical water extraction. J. Geochem. Explor. 2014, 144, 267-272. [CrossRef]

7. Plaza, M.; Amigo-Benavent, M.; del Castillo, M.D.; Ibáñez, E.; Herrero, M. Neoformation of antioxidants in glycation model systems treated under subcritical water extraction conditions. Food Res. Int. 2010, 43, 1123-1129. [CrossRef]

8. Islam, M.N.; Jung, H.Y.; Park, J.H. Subcritical water treatment of explosive and heavy metals co-contaminated soil: Removal of the explosive, and immobilization and risk assessment of heavy metals. J. Environ. Manag. 2015, 163, 262-269. [CrossRef]

9. Chemat, F.; Vian, M.A.; Cravotto, G. Green extraction of natural products: Concept and principles. Int. J. Mol. Sci. 2012, 13, 8615-8627. [CrossRef] [PubMed] 
10. Gbashi, S.; Adebo, O.A.; Piater, L.; Madala, N.E.; Njobeh, P.B. Subcritical water extraction of biological materials. Sep. Purif. Technol. 2016, 46, 21-34. [CrossRef]

11. Yang, R.F.; Zhao, C.; Chen, X.; Chan, S.W.; Wu, J.Y. Chemical properties and bioactivities of Goji (Lycium barbarum) polysaccharides extracted by different methods. J. Funct. Foods 2015, 17, 903-909. [CrossRef]

12. Ozel, M.Z.; Gogus, F.; Lewis, A.C. Subcritical water extraction of essential oils from Thymbra spicata. Food Chem. 2003, 82, 381-386. [CrossRef]

13. Xiao, S.; Xi, X.; Tang, F.; Dai, J.; Liu, J.; Lei, J.; Wang, L. Subcritical water extraction of ursolic acid from Hedyotis diffusa. Appl. Sci. 2017, 7, 187. [CrossRef]

14. Kim, W.J.; Kim, J.; Veriansyah, B.; Kim, J.D.; Lee, Y.W.; Tjandrawinata, R.R. Extraction of bioactive components from Centella asiatica using subcritical water. J. Supercrit. Fluids 2009, 48, 211-216. [CrossRef]

15. Lee, K.A.; Kim, W.J.; Kim, H.J.; Kim, K.T.; Paik, H.D. Antibacterial activity of Ginseng (Panax ginseng C. A. Meyer) stems-leaves extract produced by subcritical water extraction. Int. J. Food Sci. Technol. 2013, 48, 947-953. [CrossRef]

16. Wang, H.; Lu, Y.; Chen, J.; Li, J.; Liu, S. Subcritical water extraction of alkaloids in Sophora flavescens Ait. and determination by capillary electrophoresis with field-amplified sample stacking. J. pHarmaceut. Biomed. 2012, 58, 146-151. [CrossRef]

17. Zhang, G.; Chi, X. A green strategy for obtaining anthraquinones from Rheum tanguticum by subcritical water. Open Chem. 2020, 18, 702-710. [CrossRef]

18. Cvetanović, A.; Švarc-Gajić, J.; Gašić, U.; Tešić, Ž.; Zengin, G.; Zeković, Z.; Đurović, S. Isolation of apigenin from subcritical water extracts: Optimization of the process. J. Supercrit. Fluids 2017, 120, 32-42. [CrossRef]

19. Kim, S.W.; Ko, M.J.; Chung, M.S. Extraction of the flavonol quercetin from onion waste by combined treatment with intense pulsed light and subcritical water extraction. J. Clean. Prod. 2019, 231, 1192-1199. [CrossRef]

20. Turner, C.; Turner, P.; Jacobson, G.; Almgren, K.; Waldebäck, M.; Sjöberg, P.; Karlsson, E.N.; Markides, K.E. Subcritical water extraction and $\beta$-glucosidase-catalyzed hyrolysis of quercetin glycosides in onion waste. Green Chem. 2006, 8, 949-959. [CrossRef]

21. Esmaeelian, M.; Jahani, M.; Einafshar, S.; Feizy, J. Optimization of experimental parameters in subcritical water extraction of bioactive constituents from the saffron (Crocus sativus L.) corm based on response surface methodology. J. Food Meas. Charact. 2020, 14, 1822-1832.

22. Samadi, M.; Zainal Abidin, Z.; Yoshida, H.; Yunus, R.; Biak, D.R.A. Towards higher oil yield and quality of essential oil extracted from Aquilaria malaccensis wood via the subcritical technique. Molecules 2020, 25, 3872. [CrossRef]

23. Cho, Y.N.; Saravana, P.S.; David, N.; Chun, B.S. Biofunctional properties of wild cultivated and cultivated Ginseng (Panax ginseng Meyer) extracts obtained using subcritical water extraction. Sep. Sci. Technol. 2021, 56, 1370-1382. [CrossRef]

24. Vardanega, R.; Carvalho, P.I.N.; Santos, D.T.; Meireles, M.A.A. Obtaining prebiotic carbohydrates and beta-ecdysone from Brazilian ginseng by subcritical water extraction. Innov. Food Sci. Emerg. Technol. 2017, 42, 73-82. [CrossRef]

25. Lee, J.H.; Ko, M.J.; Chung, M.S. Subcritical water extraction of bioactive components from red ginseng (Panax ginseng C.A. Meyer). J. Supercrit. Fluids 2018, 133, 177-183. [CrossRef]

26. Zhang, Y.; Zhang, Y.; Taha, A.A.; Ying, Y.; Li, X.; Chen, X.; Ma, C. Subcritical water extraction of bioactive components from ginseng root (Panax ginseng C.A. Mey). Ind. Crop. Prod. 2018, 117, 118-127. [CrossRef]

27. Didar, Z. Co Mparative in vitro study of the biological activity and chemical composition extracts of Helicteres isora L. obtained by water and subcritical water extraction. Food Qual. Saf. 2020, 4, 101-106. [CrossRef]

28. Çam, M.; Yüksel, E.; Alaşalvar, H.; Başyiğit, B.; Şen, H.; Yilmaztekin, M.; Ahhmed, A.; Sağdiç, O. Simultaneous extraction of pHenolics and essential oil from peppermint by pressurized hot water extraction. J. Food Sci. Technol. 2019, 56, 200-207. [CrossRef]

29. Yulianto, M.; Paramita, V.; Hartati, I.; Amalia, R. Response surface methodology of pressurized liquid water extraction of curcumin from curcuma domestica val. Rasayan J. Chem. 2018, 11, 1564-1571. [CrossRef]

30. Ko, M.J.; Cheigh, C.I.; Chung, M.S. Relationship analysis between flavonoids structure and subcritical water extraction (SBWE). Food Chem. 2014, 143, 147-155. [CrossRef] [PubMed]

31. Tomšik, A.; Pavlić, B.; Vladić, J.; Cindrić, M.; Jovanov, P.; Sakač, M.; Mandić, A.; Vidović, S. Subcritical water extraction of wild garlic (Allium ursinum L.) and process optimization by response surface methodology. J. Supercrit. Fluids 2017, 128, 79-88. [CrossRef]

32. Cvetanović, A.; Zengin, G.; Zeković, Z.; Švarc-Gajić, J.; Ražić, S.; Damjanović, A.; Maškoviš, P.; Mitić, M. Co Mparative in vitro studies of the biological potential and chemical composition of stems, leaves and berries Aronia melanocarpa's extracts obtained by subcritical water extraction. Food Chem. Toxicol. 2018, 121, 458-466. [CrossRef]

33. Yu, X.M.; Zhu, P.; Zhong, Q.P.; Li, M.Y.; Ma, H.R. Subcritical water extraction of antioxidant pHenolic compounds from XiLan olive fruit dreg. J. Food Sci. Technol. 2015, 52, 5012-5020. [CrossRef]

34. Rangsriwong, P.; Rangkadilok, N.; Satayavivad, J.; Goto, M.; Shotipruk, A. Subcritical water extraction of polyphenolic compounds from Terminalia chebula Retz. fruits. Sep. Purif. Technol. 2009, 66, 51-56. [CrossRef]

35. Wang, Y.; Luan, G.; Zhou, W.; Meng, J.; Wang, H.; Hu, N.; Suo, Y. Subcritical water extraction, UPLC-Triple-TOF/MS analysis and antioxidant activity of anthocyanins from Lycium ruthenicum Murr. Food Chem. 2018, 249, 119-126. [CrossRef]

36. Kim, W.J.; Veriansyah, B.; Lee, Y.W.; Kim, J.; Kim, J.D. Extraction of mangiferin from Mahkota Dewa (Phaleria macrocarpa) using subcritical water. J. Ind. Eng. Chem. 2010, 16, 425-430. [CrossRef] 
37. Wu, H.; Li, C.; Li, Z.; Liu, R.; Zhang, A.; Xiao, Z.; Ma, L.; Li, J.; Deng, S. Simultaneous extraction of oil and tea saponin from Camellia oleifera Abel. seeds under subcritical water conditions. Fuel Process. Technol. 2018, 174, 88-94. [CrossRef]

38. Ravber, M.; Knez, Z.; Škerget, M. Simultaneous extraction of oil- and water-soluble pHase from sunflower seeds with subcritical water. Food Chem. 2015, 166, 316-323. [CrossRef] [PubMed]

39. Abdelmoez, W.; Abdelfatah, R.; Tayeb, A.; Yoshida, H. Extraction of cottonseed oil using subcritical water technology. AIChE J. 2011, 57, 2353-2359. [CrossRef]

40. Ma, X.; Jing, J.; Wang, J.; Xu, J.; Hu, Z. Extraction of low methoxyl pectin from fresh sunflower heads by subcritical water extraction. ACS Omega 2020, 5, 15095-15104. [CrossRef]

41. Kanmaz, E.Ö. Subcritical water extraction of pHenolic compounds from flaxseed meal sticks using accelerated solvent extractor (ASE). Eur. Food Res. Technol. 2013, 238, 85-91. [CrossRef]

42. Bodoira, R.; Velez, A.; Andreatta, A.E.; Martinez, M. Extraction of bioactive compounds from sesame (Sesamum indicum L.) defatted seeds using water and ethanol under sub-critical conditions. Food Chem. 2017, 237, 114-120. [CrossRef]

43. Özkaynak, E.; Ova, G. The effective parameters for subcritical water extraction of SDG lignan from flaxseed (Linum usitatissimum L.) using accelerated solvent extractor. Eur. Food Res. Technol. 2013, 237, 159-166. [CrossRef]

44. Hiep, N.T.; Duong, H.T.; Anh, D.T.; Nguyen, N.H.; Thai, D.Q.; Linh, D.; Anh, V.T.H.; Khoi, N.M. Subcritical water extraction of epigallocatechin gallate from Camellia sinensis and optimization study using response surface methodology. Processes 2020, 8, 1028. [CrossRef]

45. Lekar, A.V.; Filonova, O.V.; Borisenko, S.N.; Maksimenko, E.V.; Vetrova, E.V.; Borisenko, N.I.; minkin, V.I. Subcritical water extraction of chlorogenic acid from green coffee beans. Russ. J. pHys. Chem. B 2016, 9, 1043-1047. [CrossRef]

46. He, C.; Du, H.; Tan, C.; Chen, Z.; Chen, Z.; Yin, F.; Xu, Y.; Liu, X. Semi-continuous pressurized hot water extraction of black tea. J. Food Eng. 2018, 227, 30-41. [CrossRef]

47. Rodríguez-Meizoso, I.; Marin, F.R.; Herrero, M.; Señorans, F.J.; Reglero, G.; Cifuentes, A.; Ibáñez, E. Subcritical water extraction of nutraceuticals with antioxidant activity from oregano. chemical and functional characterization. J. pHarm. Biomed. Anal. 2006, 41, 1560-1565. [CrossRef] [PubMed]

48. Yang, Y.; Kayan, B.; Bozer, N.; Pate, B.; Baker, C.; Gizir, A.M. Terpene degradation and extraction from basil and oregano leaves using subcritical water. J. Chromatogr. A 2007, 1152, 262-267. [CrossRef] [PubMed]

49. Švarc-Gajić, J.; Cerdà, V.; Clavijo, S.; Suárez, R.; Zengin, G.; Cvetanoviá, A. Chemical and bioactivity screening of subcritical water extracts of chokeberry (Aronia melanocarpa) stems. J. pHarm. Biomed. Anal. 2019, 164, 353-359. [CrossRef] [PubMed]

50. Lachos-Perez, D.; Baseggio, A.M.; Mayanga-Torres, P.C.; Maróstica, M.R.; Rostagno, M.A.; Martínez, J.; Forster-Carneiro, T. Subcritical water extraction of flavanones from defatted orange peel. J. Supercrit. Fluids 2018, 138, 7-16. [CrossRef]

51. Ho, B.C.H.; Kamal, S.M.M.; Danquah, M.K.; Harun, R. Optimization of subcritical water extraction (SBWE) of lipid and eicosapentaenoic acid (EPA) from nannochloropsis gaditana. BioMed Res. Int. 2018, 2018, 8273581. [CrossRef]

52. Vo Dinh, T.; Saravana, P.S.; Woo, H.C.; Chun, B.S. Ionic liquid-assisted subcritical water enhances the extraction of pHenolics from brown seaweed and its antioxidant activity. Sep. Purif. Technol. 2018, 196, 287-299. [CrossRef]

53. Rodríguez-Meizoso, I.; Jaime, L.; Santoyo, S.; Seíoráns, F.J.; Cifuentes, A.; Ibáñez, E. Subcritical water extraction and characterization of bioactive compounds from Haematococcus pluvialis microalga. J. pHarm. Biomed. Anal. 2010, 51, 456-463. [CrossRef] [PubMed]

54. Chikari, F.; Han, J.; Wang, Y.; Ao, W. Synergized subcritical-ultrasound assisted aqueous two-phase extraction, purification, and characterization of Lentinus edodes polysaccharides. Process Biochem. 2020, 95, 297-306. [CrossRef]

55. Zhang, J.; Wen, C.; Gu, J.; Ji, C.; Duan, Y.; Zhang, H. Effects of subcritical water extraction microenvironment on the structure and biological activities of polysaccharides from Lentinus edodes. Int. J. Biol. Macromol. 2019, 123, 1002-1011. [CrossRef] [PubMed]

56. Zhang, J.; Wen, C.; Qin, W.; Qin, P.; Zhang, H.; Duan, Y. Ultrasonic-enhanced subcritical water extraction of polysaccharides by two steps and its characterization from Lentinus edodes. Int. J. Biol. Macromol. 2018, 118, 2269-2277. [CrossRef]

57. Morales, D.; Smiderle, F.R.; Villalva, M.; Abreu, H.; Rico, C.; Santoyo, S.; Iacomini, M.; Soler-Rivas, C. Testing the effect of combining innovative extraction technologies on the biological activities of obtained $\beta$-glucan-enriched fractions from Lentinula edodes. J. Funct. Foods 2019, 60, 103446. [CrossRef]

58. Yang, L.; Qu, H.; Mao, G.; Zhao, T.; Li, F.; Zhu, B.; Zhang, B.; Wu, X. Optimization of subcritical water extraction of polysaccharides from Grifola frondosa using response surface methodology. Pharmacogn. Mag. 2013, 9, 120-129.

59. Zhang, J.; Wen, C.; Chen, M.; Gu, J.; Zhou, J.; Duan, Y.; Zhang, H.; Ma, H. Antioxidant activities of Sagittaria sagittifolia L. polysaccharides with subcritical water extraction. Int. J. Biol. Macromol. 2019, 134, 172-179. [CrossRef] [PubMed]

60. Gu, J.; Zhang, H.; Yao, H.; Zhou, J.; Duan, Y.; Ma, H. Co Mparison of characterization, antioxidant and immunological activities of three polysaccharides from Sagittaria sagittifolia L. Carbohydr. Polym. 2020, 235, 115939. [CrossRef]

61. Zhang, J.; Chen, M.; Wen, C.; Zhou, J.; Gu, J.; Duan, Y.; Zhang, H.; Ren, X.; Ma, H. Structural characterization and immunostimulatory activity of a novel polysaccharide isolated with subcritical water from Sagittaria sagittifolia L. Int. J. Biol. Macromol. 2019, 133, 11-20. [CrossRef] [PubMed]

62. Luo, X.; Duan, Y.; Yang, W.; Zhang, H.; Li, C.; Zhang, J. Structural elucidation and immunostimulatory activity of polysaccharide isolated by subcritical water extraction from Cordyceps militaris. Carbohydr. Polym. 2017, 157, 794-802. [CrossRef]

63. Deshpande, G.V.; Holder, G.D.; Bishop, A.A.; Gopal, J.; Wender, I. Extraction of coal using supercritical water. Fuel 1984, 63, 956-960. [CrossRef] 
64. Calderon, N.B.J. Extraction of oil sand bitumens with supercritical water. Fuel Process. Technol. 1990, $25,33-44$.

65. Helling, R.K.; Tester, J.W. Oxidation kinetics of carbon monoxide in supercritical water. Energy Fuels 1987, 1, 417-423. [CrossRef]

66. Hawthorne, S.B.; Yang, Y.; Miller, D.J. Extraction of organic pollutants from environmental solids with sub- and supercritical water. Anal. Chem. 1994, 66, 2912-2920. [CrossRef]

67. Yang, Y.; Bowadt, S.; Hawthorne, S.B.; Miller, D.J. Subcritical water extraction of polychlorinated biphenyls from soil and sediment. Anal. Chem. 1995, 67, 4571-4576. [CrossRef]

68. Li, B.; Yang, Y. Subcritical water extraction coupled to high-performance liquid chromatography. Anal. Chem. 1999, 71, 1491-1495.

69. Li, B.; Gan, Y.; Eaton, C.D.; He, P.; Jones, A.D. On-line coupling of subcritical water extraction with high-performance liquid chromatography via solid-phase trapping. J. Chromatogr. A 2000, 873, 175-184. [CrossRef]

70. Lamm, L.; Yang, Y. Off-line coupling of subcritical water extraction with subcritical water chromatography via a sorbent trap and thermal desorption. Anal. Chem. 2003, 75, 2237-2242. [CrossRef]

71. Chen, H.M.; Fu, X.; Luo, Z.G. Properties and extraction of pectin-enriched materials from sugar beet pulp by ultrasonic-assisted treatment combined with subcritical water. Food Chem. 2015, 168, 302-310. [CrossRef]

72. Yang, Z.; Uhler, B.; Lipkie, T. Microwave-assisted subcritical water extraction of steviol glycosides from stevia rebaudiana leaves. Nat. Prod. Commun. 2019, 14, 1-4. [CrossRef]

73. Espley, R.V.; Butts, C.A.; Laing, W.A.; Martell, S.; Smith, H.; McGhie, T.K.; Zhang, J.; Paturi, G.; Hedderley, D.; Bovy, A.G.; et al. Dietary flavonoids from modified apple reduce inflammation markers and modulate gut microbiota in mice. J. Nutr. 2014, 144, 146-154. [CrossRef]

74. Ko, M.J.; Kwon, H.L.; Chung, M.S. Pilot-scale subcritical water extraction of flavonoids from satsuma mandarin (Citrus unshiu Markovich) peel. Innov. Food Sci. Emerg. Technol. 2016, 38, 175-181. [CrossRef]

75. Lee, K.A.; Kim, K.T.; Kim, H.J.; Chung, M.S.; Chang, P.S.; Park, H.; Pai, H.D. Antioxidant activities of onion (Allium cepa L.) peel extracts produced by ethanol, hot water, and subcritical water extraction. Food Sci. Biotechnol. 2014, 23, 615-621. [CrossRef]

76. Kumar, M.S.; Dutta, R.; Prasad, D.; Misra, K. Subcritical water extraction of antioxidant compounds from Seabuckthorn (Hippophae rhamnoides) leaves for the co Mparative evaluation of antioxidant activity. Food Chem. 2011, 127, 1309-1316. [CrossRef] [PubMed]

77. Munir, M.T.; Kheirkhah, H.; Baroutian, S.; Quek, S.Y.; Young, B.R. Subcritical water extraction of bioactive compounds from waste onion skin. J. Clean. Prod. 2018, 183, 487-494. [CrossRef]

78. Vladić, J.; Jakovljević, M.; Molnar, M.; Vidović, S.; Tomić, M.; Drinić, Z.; Jokić, S. Valorization of yarrow (Achillea millefolium L.) by-product through application of subcritical water extraction. Molecules 2020, 25, 1878. [CrossRef]

79. Zabidi, N.A.; Ishak, N.A.; Hamid, M.; Ashari, S.E. Subcritical water extraction of antioxidants from Curculigo latifolia root. J. Chem. 2019, 2019, 1-10. [CrossRef]

80. Hwang, H.J.; Kim, H.J.; Ko, M.J.; Chung, M.S. Recovery of hesperidin and narirutin from waste citrus unshiu peel using subcritical water extraction aided by pulsed electric field treatment. Food Sci. Biotechnol. 2021, 30, 217-226. [CrossRef]

81. Nkurunziza, D.; Pendleton, P.; Chun, B.S. Optimization and kinetics modeling of okara isoflavones extraction using subcritical water. Food Chem. 2019, 295, 613-621. [CrossRef]

82. Ko, M.J.; Cheigh, C.I.; Cho, S.W.; Chung, M.S. Subcritical water extraction of flavonol quercetin from onion skin. J. Food Eng. 2011, 102, 327-333. [CrossRef]

83. Zhang, H.; Liu, S.; Li, H.; Xue, F.; Han, S.; Wang, L.; Cheng, Y.; Wang, X. Extraction of isoflavones from Puerariae lobata using subcritical water. RSC Adv. 2018, 8, 22652-22658. [CrossRef]

84. Zeković, Z.; Vidović, S.; Vladić, J.; Radosavljević, R.; Cvejin, A.; Elgndi, M.A.; Pavlić, B. Optimization of subcritical water extraction of antioxidants from Coriandrum sativum seeds by response surface methodology. J. Supercrit. Fluids 2014, 95, 560-566. [CrossRef]

85. Kim, D.S.; Lim, S.B. Kinetic study of subcritical water extraction of flavonoids from citrus unshiu peel. Sep. Purif. Technol. 2020, 250, 117259. [CrossRef]

86. Ko, M.J.; Lee, J.H.; Nam, H.H.; Chung, M.S. Subcritical water extraction of pHytochemicals from Phlomis umbrosa Turcz. Innov. Food Sci. Emerg. Technol. 2017, 42, 1-7. [CrossRef]

87. Guthrie, F.; Wang, Y.; Neeve, N.; Quek, S.Y.; Mohammadi, K.; Baroutian, S. Recovery of pHenolic antioxidants from green kiwifruit peel using subcritical water extraction. Food Bioprod. Process. 2020, 122, 136-144. [CrossRef]

88. Cheng, Y.; Qu, S.; Wang, Z.; Xue, F.; Li, F. Controlled extraction of flavonoids from Radix Scutellariae by subcritical water. Clean Soil Air Water 2016, 44, 299-303. [CrossRef]

89. Kim, J.W.; Nagaoka, T.; Ishida, Y.; Hasegawa, T.; Kitagawa, K.; Lee, S.C. Subcritical water extraction of nutraceutical compounds from citrus pomaces. Sep. Sci. Technol. 2009, 44, 2598-2608. [CrossRef]

90. Kim, D.S.; Lim, S.B. Semi-continuous subcritical water extraction of flavonoids from Citrus unshiu peel: Their antioxidant and enzyme inhibitory activities. Antioxidants 2020, 9, 360. [CrossRef]

91. Cheigh, C.I.; Chung, E.Y.; Chung, M.S. Enhanced extraction of flavanones hesperidin and narirutin from Citrus unshiu peel using subcritical water. J. Food Eng. 2012, 110, 472-477. [CrossRef]

92. Ho, T.C.; Chun, B.S. Extraction of bioactive compounds from pseuderanthemum palatiferum (nees) radlk. using subcritical water and conventional solvents: A co Mparison study. J. Food Sci. 2019, 84, 1201-1207. [CrossRef] 
93. Fan, R.; Xiang, J.; Li, N.; Jiang, X.; Gao, Y. I Mpact of extraction parameters on chemical composition and antioxidant activity of bioactive compounds from Chinese licorice (Glycyrrhiza uralensis Fisch.) by subcritical water. Sep. Sci. Technol. 2015, 51, 609-621. [CrossRef]

94. Xu, H.; Wang, W.; Jiang, J.; Yuan, F.; Gao, Y. Subcritical water extraction and antioxidant activity evaluation with on-line HPLC-ABTS(·+) assay of pHenolic compounds from marigold (Tagetes erecta L.) flower residues. J. Food Sci. Technol. 2015, 52, 3803-3811. [CrossRef]

95. Song, R.; Ismail, M.; Baroutian, S.; Farid, M. Effect of subcritical water on the extraction of bioactive compounds from carrot leaves. Food Bioprocess Technol. 2018, 11, 1895-1903. [CrossRef]

96. Cvetanović, A.; Švarc-Gajić, J.; Mašković, P.; Savić, S.; Nikolić, L. Antioxidant and biological activity of chamomile extracts obtained by different techniques: Perspective of using superheated water for isolation of biologically active compounds. Ind. Crop. Prod. 2015, 65, 582-591. [CrossRef]

97. Platonov, I.A.; Nikitchenko, N.V.; Onuchak, L.A.; Arutyunov, Y.I.; Kurkin, V.A.; Smirnov, P.V. Subcritical water extraction of biologically active substances from milk thistle seed (Silybum murianum L.). Russ. J. pHys. Chem. B 2011, 4, 1211-1216. [CrossRef]

98. Vidović, S.; Nastić, N.; Gavarić, A.; Cindrić, M.; Vladić, J. Development of green extraction process to produce antioxidant-rich extracts from purple coneflower. Sep. Sci. Technol. 2018, 54, 1174-1181. [CrossRef]

99. Gil-Ramírez, A.; Mendiola, J.A.; Arranz, E.; Ruíz-Rodríguez, A.; Reglero, G.; Ibáñez, E.; Marín, F.R. Highly isoxanthohumol enriched hop extract obtained by pressurized hot water extraction (PHWE). Chemical and functional characterization. Innov. Food Sci. Emerg. Technol. 2012, 16, 54-60. [CrossRef]

100. Essien, S.; Young, B.; Baroutian, S. Subcritical water extraction for selective recovery of pHenolic bioactives from kānuka leaves. J. Supercrit. Fluids 2020, 158, 104721. [CrossRef]

101. Shaddel, R.; Maskooki, A.; Haddad-Khodaparast, M.H.; Azadmard-Damirchi, S.; Mohamadi, M.; Fathi-Achachlouei, B. Optimization of extraction process of bioactive compounds from Bene hull using subcritical water. Food Sci. Biotechnol. 2014, 23, 1459-1468. [CrossRef]

102. Mašković, P.; Veličković, V.; Mitić, M.; Đurović, S.; Zeković, Z.; Radojković, M.; Cvetanović, A.; Švarc-Gajić, J.; Vujić, J. Summer savory extracts prepared by novel extraction methods resulted in enhanced biological activity. Ind. Crop. Prod. 2017, 109, 875-881. [CrossRef]

103. Zeković, Z.; Cvetanović, A.; Švarc-Gajić, J.; Gorjanović, S.; Sužnjević, D.; Mašković, P.; Savić, S.; Radojković, M.; Đurović, S. Chemical and biological screening of stinging nettle leaves extracts obtained by modern extraction techniques. Ind. Crop. Prod. 2017, 108, 423-430. [CrossRef]

104. Pavlova, L.V.; Platonov, I.A.; Kurkin, V.A.; Afanasyeva, P.V.; Novikova, E.A.; Mukhanova, I.M. Evaluation of the extraction efficiency of biologically active compounds from chamomile flowers (Chamomilla recutita R.) grown in the Samara region by extractants in the subcritical state. Russ. J. pHys. Chem. B 2019, 12, 1212-1224. [CrossRef]

105. Nkurunziza, D.; Pendleton, P.; Sivagnanam, S.P.; Park, J.S.; Chun, B.S. Subcritical water enhances hydrolytic conversions of isoflavones and recovery of pHenolic antioxidants from soybean byproducts (okara). J. Ind. Eng. Chem. 2019, 80, 696-703. [CrossRef]

106. Huaman-Castilla, N.L.; Mariotti-Celis, M.S.; Martinez-Cifuentes, M.; Perez-Correa, J.R. Glycerol as alternative co-solvent for water extraction of polyphenols from carmenere pomace: Hot pressurized liquid extraction and computational chemistry calculations. Biomolecules 2020, 10, 474. [CrossRef]

107. Švarc-Gajić, J.; Cvetanović, A.; Segura-Carretero, A.; Mašković, P.; Jakšić, A. Functional coffee substitute prepared from ginger by subcritical water. J. Supercrit. Fluids 2017, 128, 32-38. [CrossRef]

108. Khoza, B.S.; Dubery, I.A.; Byth-Illing, H.A.; Steenkamp, P.A.; Chimuka, L.; Madala, N.E. Optimization of pressurized hot water extraction of flavonoids from Momordica foetida using UHPLC-qTOF-MS and multivariate chemometric approaches. Food Anal. Methods 2015, 9, 1480-1489. [CrossRef]

109. Vladić, J.; Janković, T.; Živković, J.; Tomić, M.; Zdunić, G.; Šavikin, K.; Vidović, S. Co Mparative study of subcritical water and microwave-assisted extraction techniques i Mpact on the pHenolic compounds and 5-hydroxymethylfurfural content in pomegranate peel. Plant Foods Hum. Nutr. 2020, 75, 553-560. [CrossRef] [PubMed]

110. Pinto, D.; Vieira, E.F.; Peixoto, A.F.; Freire, C.; Freitas, V.; Costa, P.; Delerue-Matos, C.; Rodrigues, F. Optimizing the extraction of pHenolic antioxidants from chestnut shells by subcritical water extraction using response surface methodology. Food Chem. 2021, 334, 127521. [CrossRef] [PubMed]

111. Pavlić, B.; Vidović, S.; Vladić, J.; Radosavljević, R.; Cindrić, M.; Zeković, Z. Subcritical water extraction of sage (Salvia officinalis L.) by-products—Process optimization by response surface methodology. J. Supercrit. Fluids 2016, 116, 36-45. [CrossRef]

112. Ersan, S.; Ustundag, O.G.; Carle, R.; Schweiggert, R.M. Subcritical water extraction of pHenolic and antioxidant constituents from pistachio (Pistacia vera L.) hulls. Food Chem. 2018, 253, 46-54. [CrossRef]

113. Ko, M.J.; Nam, H.H.; Chung, M.S. Conversion of 6-gingerol to 6-shogaol in ginger (Zingiber officinale) pulp and peel during subcritical water extraction. Food Chem. 2019, 270, 149-155. [CrossRef]

114. Luo, X.; Cui, J.; Zhang, H.; Duan, Y. Subcritical water extraction of polyphenolic compounds from sorghum (Sorghum bicolor L.) bran and their biological activities. Food Chem. 2018, 262, 14-20. [CrossRef] [PubMed]

115. Yan, Z.; Luo, X.; Cong, J.; Zhang, H.; Ma, H.; Duan, Y. Subcritical water extraction, identification and antiproliferation ability on HepG2 of polyphenols from lotus seed epicarp. Ind. Crop. Prod. 2019, 129, 472-479. [CrossRef] 
116. Cvetanović, A.; Švarc-Gajić, J.; Zeković, Z.; Gašić, U.; Tešić, Z.; Zengin, G.; Mašković, P.; Mahomoodally, M.F.; Đurović, S. Subcritical water extraction as a cutting edge technology for the extraction of bioactive compounds from chamomile: Influence of pressure on chemical composition and bioactivity of extracts. Food Chem. 2018, 266, 389-396. [CrossRef]

117. Dzah, C.S.; Duan, Y.; Zhang, H.; Authur, D.A.; Ma, H. Ultrasound-, subcritical water- and ultrasound assisted subcritical water-derived Tartary buckwheat polyphenols show superior antioxidant activity and cytotoxicity in human liver carcinoma cells. Food Res. Int. 2020, 137, 109598. [CrossRef] [PubMed]

118. Naffati, A.; Vladić, J.; Pavlić, B.; Radosavljević, R.; Gavarić, A.; Vidović, S. Recycling of filter tea industry by-products: Application of subcritical water extraction for recovery of bioactive compounds from A. uva-ursi herbal dust. J. Supercrit. Fluids 2017, 121, 1-9. [CrossRef]

119. Gong, Y.; Zhang, X.; He, L.; Yan, Q.; Yuan, F.; Gao, Y. Optimization of subcritical water extraction parameters of antioxidant polyphenols from sea buckthorn (Hippophae rhamnoides L.) seed residue. J. Food Sci. Technol. 2015, 52, 1534-1542. [CrossRef] [PubMed]

120. Aliakbarian, B.; Fathi, A.; Perego, P.; Dehghani, F. Extraction of antioxidants from winery wastes using subcritical water. J. Supercrit. Fluids 2012, 65, 18-24. [CrossRef]

121. Cvetanović, A.; Švarc-Gajić, J.; Zeković, Z.; Jerković, J.; Zengin, G.; Gašić, U.; Tešić, Z.; Mašković, P.; Soares, C.; Barroso, M.F.; et al. The influence of the extraction temperature on polyphenolic profiles and bioactivity of chamomile (Matricaria chamomilla L.) subcritical water extracts. Food Chem. 2019, 271, 328-337. [CrossRef]

122. Yan, Z.; Zhang, H.; Dzah, C.S.; Zhang, J.; Diao, C.; Ma, H.; Duan, Y. Subcritical water extraction, identification, antioxidant and antiproliferative activity of polyphenols from lotus seedpod. Sep. Purif. Technol. 2020, 236, 116217. [CrossRef]

123. Loarce, L.; Oliver-Simancas, R.; Marchante, L.; Díaz-Maroto, M.C.; Alañòn, M.E. Implementation of subcritical water extraction with natural deep eutectic solvents for sustainable extraction of pHenolic compounds from winemaking by-products. Food Res. Int. 2020, 137, 109728. [CrossRef]

124. Gagić, T.; Knez, Z.; Škerget, M. Subcritical water extraction of chestnut bark and optimization of process parameters. Molecules 2020, 25, 2774. [CrossRef]

125. Vladic, J.; Nastic, N.; Stanojkovic, T.; Zizak, Z.; Cakarevic, J.; Popovic, L.; Vidovic, S. Subcritical water for recovery of polyphenols from comfrey root and biological activities of extracts. Acta Chim. Slov. 2019, 66, 473-783. [CrossRef] [PubMed]

126. Duba, K.S.; Casazza, A.A.; Mohamed, H.B.; Perego, P.; Fiori, L. Extraction of polyphenols from grape skins and defatted grape seeds using subcritical water: Experiments and modeling. Food Bioprod. Process 2015, 94, 29-38. [CrossRef]

127. Xu, H.; Wang, W.; Liu, X.; Yuan, F.; Gao, Y. Antioxidative pHenolics obtained from spent coffee grounds (Coffea arabica L.) by subcritical water extraction. Ind. Crop. Prod. 2015, 76, 946-954. [CrossRef]

128. Kiamahalleh, M.V.; Najafpour-Darzi, G.; Rahimnejad, M.; Moghadamnia, A.A.; Kiamahalleh, M.V. High performance curcumin subcritical water extraction from turmeric (Curcuma longa L.). J. Chromatogr. B Analyt. Technol. Biomed. Life Sci. 2016, 1022, 191-198. [CrossRef] [PubMed]

129. Mottahedin, P.; Asl, A.H.; Khajenoori, M. Extraction of curcumin and essential oil from Curcuma longa L. by subcritical water via response surface methodology. J. Food Process. Preserv. 2017, 41, e13095. [CrossRef]

130. Kwon, H.; Chung, M. Pilot-scale subcritical solvent extraction of curcuminoids from Curcuma long L. Food Chem. 2015, 185, 58-64. [CrossRef]

131. Euterpio, M.A.; Cavaliere, C.; Capriotti, A.L.; Crescenzi, C. Extending the applicability of pressurized hot water extraction to compounds exhibiting limited water solubility by $\mathrm{pH}$ control: Curcumin from the turmeric rhizome. Anal. Bioanal. Chem. 2011, 401, 2977-2985. [CrossRef]

132. Budrat, P.; Shotipruk, A. Enhanced recovery of pHenolic compounds from bitter melon (Momordica charantia) by subcritical water extraction. Sep. Purif. Technol. 2009, 66, 125-129. [CrossRef]

133. Nastić, N.; Švarc-Gajić, J.; Delerue-Matos, C.; Barroso, M.F.; Soares, C.; Moreira, M.M.; Morais, S.; Mašković, P.; Srček, V.G.; Slivac, I.; et al. Subcritical water extraction as an environmentally-friendly technique to recover bioactive compounds from traditional Serbian medicinal plants. Ind. Crop. Prod. 2018, 111, 579-589. [CrossRef]

134. Švarc-Gajić, J.; Cerdà, V.; Clavijo, S.; Suárez, R.; Mašković, P.; Cvetanović, A.; Delerue-Matos, C.; Carvalho, A.P.; Novakov, V. Bioactive compounds of sweet and sour cherry stems obtained by subcritical water extraction. J. Chem. Technol. Biotechnol. 2018, 93, 1627-1635. [CrossRef]

135. Gagić, T.; Knez, Ž.; Škerget, M. Hydrothermal hydrolysis of sweet chestnut (Castanea sativa) tannins. J. Serb. Chem. Soc. 2020, 85, 867-881. [CrossRef]

136. Singh, P.P.; Saldaña, M.D.A. Subcritical water extraction of pHenolic compounds from potato peel. Food Res. Int. 2011, 44, 2452-2458. [CrossRef]

137. Kheirkhah, H.; Baroutian, S.; Quek, S.Y. Evaluation of bioactive compounds extracted from Hayward kiwifruit pomace by subcritical water extraction. Food Bioprod. Process. 2019, 115, 143-153. [CrossRef]

138. Pangestuti, R.; Getachew, A.T.; Siahaan, E.A.; Chun, B.S. Characterization of functional materials derived from tropical red seaweed Hypnea musciformis produced by subcritical water extraction systems. J. Appl. Psychol. 2019, 31, 2517-2528. [CrossRef]

139. Rodrigues, L.G.G.; Mazzutti, S.; Vitali, L.; Micke, G.A.; Ferreira, S.R.S. Recovery of bioactive pHenolic compounds from papaya seeds agroindustrial residue using subcritical water extraction. Biocatal. Agric. Biotechnol. 2019, 22, 101367. [CrossRef] 
140. Švarc-Gajić, J.; Cvetanović, A.; Segura-Carretero, A.; Linares, I.B.; Mašković, P. Characterisation of ginger extracts obtained by subcritical water. J. Supercrit. Fluids 2017, 123, 92-100. [CrossRef]

141. Zakaria, S.M.; Kamal, S.M.M.; Harun, M.R.; Omar, R.; Siajam, S.I. Subcritical water technology for extraction of pHenolic compounds from Chlorella sp. microalgae and assessment on its antioxidant activity. Molecules 2017, 22, 1092. [CrossRef]

142. Dorosh, O.; Moreira, M.M.; Pinto, D.; Freire, C.; Costa, P.; Rodrigues, F.; Delerue-Matos, C. Evaluation of the extraction temperature influence on polyphenolic profiles of vine-canes (Vitis vinifera) subcritical water extracts. Foods 2020, 9, 872. [CrossRef]

143. Cha, J.; Kim, C.T.; Kim, T.E.; Cho, Y.J. Optimization of subcritical extraction process for cinnamon (Cinnamomum Cassia Blume) using response surface methodology. Food Sci. Biotechnol. 2019, 28, 1703-1711. [CrossRef] [PubMed]

144. He, L.; Zhang, X.; Xu, H.; Xu, C.; Yuan, F.; Knez, Ž.; Novak, Z.; Gao, Y. Subcritical water extraction of pHenolic compounds from pomegranate (Punica granatum L.) seed residues and investigation into their antioxidant activities with HPLC-ABTS+ assay. Food Bioprod. Process. 2012, 90, 215-223. [CrossRef]

145. Nastić, N.; Švarc-Gajić, J.; Delerue-Matos, C.; Morais, S.; Barroso, M.F.; Moreira, M.M. Subcritical water extraction of antioxidants from mountain germander (Teucrium montanum L.). J. Supercrit. Fluids 2018, 138, 200-206. [CrossRef]

146. Wu, Y.; Jiang, Y.; Zhang, L.; Zhou, J.; Yu, Y.; Zhang, S.; Zhou, Y. Green and efficient extraction of total glucosides from Paeonia lactiflora Pall. 'Zhongjiang' by subcritical water extraction combined with macroporous resin enrichment. Ind. Crop. Prod. 2019, 141, 111699. [CrossRef]

147. Koyu, H.; Kazan, A.; Ozturk, T.K.; Yesil-Celiktas, O.; Haznedaroglu, M.Z. Optimizing subcritical water extraction of Morus nigra L. fruits for maximization of tyrosinase inhibitory activity. J. Supercrit. Fluids 2017, 127, 15-22. [CrossRef]

148. Yildiz-Ozturk, E.; Tag, O.; Yesil-Celiktas, O. Subcritical water extraction of steviol glycosides from Stevia rebaudiana leaves and characterization of the raffinate pHase. J. Supercrit. Fluids 2014, 95, 422-430. [CrossRef]

149. Meng, F.; Cheng, Y. Subcritical water extraction of pHenolic compounds and analysis of inorganic elements from Erigeron breviscapus. ChemistrySelect 2019, 4, 7173-7180. [CrossRef]

150. Fernández-Ponce, M.T.; Casas, L.; Mantell, C.; Rodríguez, M.; de la Ossa, E.M. Extraction of antioxidant compounds from different varieties of Mangifera indica leaves using green technologies. J. Supercrit. Fluids 2012, 72, 168-175. [CrossRef]

151. Sarfarazi, M.; Jafari, S.M.; Rajabzadeh, G.; Feizi, J. Development of an environmentally-friendly solvent-free extraction of saffron bioactives using subcritical water. LWT 2019, 114, 108428. [CrossRef]

152. Baek, J.Y.; Lee, J.M.; Lee, S.C. Extraction of nutraceutical compounds from licorice root with subcritical water. Sep. Purif. Technol. 2008, 63, 661-664. [CrossRef]

153. Yilmaz-Turan, S.; Jimenez-Quero, A.; Moriana, R.; Arte, E.; Katina, K.; Vilaplana, F. Cascade extraction of proteins and feruloylated arabinoxylans from wheat bran. Food Chem. 2020, 333, 127491. [CrossRef] [PubMed]

154. Saravana, P.S.; Tilahun, A.; Gerenew, C.; Tri, V.D.; Kim, N.H.; Kim, G.D.; Woo, H.C.; Chun, B.S. Subcritical water extraction of fucoidan from Saccharina japonica: Optimization, characterization and biological studies. J. Appl. pHycol. 2017, 30, 79-590. [CrossRef]

155. Liew, S.Q.; Teoh, W.H.; Tan, C.K.; Yusoff, R.; Ngoh, G.C. Subcritical water extraction of low methoxyl pectin from pomelo (Citrus grandis (L.) Osbeck) peels. Int. J. Biol. Macromol. 2018, 116, 128-135. [CrossRef] [PubMed]

156. Munoz-Almagro, N.; Valadez-Carmona, L.; Mendiola, J.A.; Ibanez, E.; Villamiel, M. Structural characterisation of pectin obtained from cacao pod husk. co Mparison of conventional and subcritical water extraction. Carbohydr. Polym. 2019, 217, 69-78. [CrossRef]

157. Gereniu, C.R.N.; Saravana, P.S.; Chun, B.S. Recovery of carrageenan from Solomon Islands red seaweed using ionic liquid-assisted subcritical water extraction. Sep. Purif. Technol. 2018, 196, 309-317. [CrossRef]

158. Ho, T.C.; Kiddane, A.T.; Sivagnanam, S.P.; Park, J.S.; Cho, Y.J.; Getachew, A.; Nguyen, T.T.; Kim, G.D.; Chun, B.S. Green extraction of polyphenolic-polysaccharide conjugates from Pseuderanthemum palatiferum (Nees) Radlk.: Chemical profile and anticoagulant activity. Int. J. Biol. Macromol. 2020, 157, 484-493. [CrossRef]

159. Yan, J.K.; Wu, L.X.; Cai, W.D.; Xiao, G.S.; Duan, Y.; Zhang, H. Subcritical water extraction-based methods affect the pHysicochemical and functional properties of soluble dietary fibers from wheat bran. Food Chem. 2019, 298, 124987. [CrossRef]

160. Chao, Z.; Ri, Y.; Tai, Q. Ultrasound-enhanced subcritical water extraction of polysaccharides from Lycium barbarum L. Sep. Purif. Technol. 2013, 120, 141-147. [CrossRef]

161. Du, X.; Bai, X.; Gao, W.; Jiang, Z. Properties of soluble dietary fibre from defatted coconut flour obtained through subcritical water extraction. Int. J. Food Sci. Technol. 2019, 54, 1390-1404. [CrossRef]

162. Sun, H.; Yuan, X.; Zhang, Z.; Su, X.; Shi, M. Thermal processing effects on the chemical constituent and antioxidant activity of Okara extracts using subcritical water extraction. J. Chem. 2018, 2018, 1-8. [CrossRef]

163. Saravana, P.S.; Cho, Y.N.; Woo, H.C.; Chun, B.S. Green and efficient extraction of polysaccharides from brown seaweed by adding deep eutectic solvent in subcritical water hydrolysis. J. Clean. Prod. 2018, 198, 1474-1484. [CrossRef]

164. Klinchongkon, K.; Chanthong, N.; Ruchain, K.; Khuwijitjaru, P.; Adachi, S. Effect of ethanol addition on subcritical water extraction of pectic polysaccharides from Passion fruit peel. J. Food Process. Preserv. 2017, 41, e13138. [CrossRef]

165. Plaza, M.; Amigo-Benavent, M.; Castillo, M.D.; Ibáñez, E.; Herrero, M. Facts about the formation of new antioxidants in natural samples after subcritical water extraction. Food Res. Int. 2010, 43, 2341-2348. [CrossRef]

166. Viriya-Empikul, J.W.N.; Takashi, K.; Shuji, A. Effects of temperature and flow rate on subcritical-water extraction from defatted rice bran. Food Sci. Technol. Res. 2012, 18, 333-340. [CrossRef] 
167. Alboofetileh, M.; Rezaei, M.; Tabarsa, M.; You, S.; Mariatti, F.; Cravotto, G. Subcritical water extraction as an efficient technique to isolate biologically-active fucoidans from Nizamuddinia zanardinii. Int. J. Biol. Macromol. 2019, 128, 244-253. [CrossRef] [PubMed]

168. Liu, J.; Li, Y.; Liu, W.; Qi, Q.; Hu, X.; Li, S.; Lei, J.; Rong, L. Extraction of polysaccharide from dendrobium nobile Lindl. by subcritical water extraction. ACS Omega 2019, 4, 20586-20594. [CrossRef] [PubMed]

169. Bordoloi, A.; Goosen, N.J. A greener alternative using subcritical water extraction to valorize the brown macroalgae Ecklonia maxima for bioactive compounds. J. Appl. pHycol. 2020, 32, 2307-2319. [CrossRef]

170. Pedras, B.; Salema-Oom, M.; Sá-Nogueira, I.; Simões, P.; Paiva, A.; Barreiros, S. Valorization of white wine grape pomace through application of subcritical water: Analysis of extraction, hydrolysis, and biological activity of the extracts obtained. J. Supercrit. Fluids 2017, 128, 138-144. [CrossRef]

171. Pedras, B.M.; Nascimento, M.; Sá-Nogueira, I.; Simões, P.; Paiva, A.; Barreiros, S. Semi-continuous extraction/hydrolysis of spent coffee grounds with subcritical water. J. Ind. Eng. Chem. 2019, 72, 453-456. [CrossRef]

172. Limsangouan, N.; Milasing, N.; Thongngam, M.; Khuwijitjaru, P.; Jittanit, W. pHysical and chemical properties, antioxidant capacity, and total pHenolic content of xyloglucan component in tamarind (Tamarindus indica) seed extracted using subcritical water. J. Food Process. Preserv. 2019, 43, 1-10. [CrossRef]

173. Nomura, S.; Lee, W.J.; Konishi, M.; Saitoh, T.; Murata, M.; Ohtsu, N.; Shimotori, Y.; Kohari, Y.; Nagata, Y.; Chiou, T.Y. Characteristics of Japanese mint extracts obtained by subcritical-water treatment. Food Sci. Technol. Res. 2019, 25, 695-703. [CrossRef]

174. Eikani, M.H.; Golmohammad, F.; Rowshanzamir, S. Subcritical water extraction of essential oils from coriander seeds (Coriandrum sativum L.). J. Food Eng. 2007, 80, 735-740. [CrossRef]

175. Zeković, Z.; Kaplan, M.; Pavlić, B.; Olgun, E.O.; Vladić, J.; Canlı, O.; Vidović, S. Chemical characterization of polyphenols and volatile fraction of coriander (Coriandrum sativum L.) extracts obtained by subcritical water extraction. Ind. Crop. Prod. 2016, 87, 54-63. [CrossRef]

176. Ma, Q.; Fan, X.D.; Liu, X.C.; Qiu, T.Q.; Jiang, J.G. Ultrasound-enhanced subcritical water extraction of essential oils from Kaempferia galangal L. and their co Mparative antioxidant activities. Sep. Purif. Technol. 2015, 150, 73-79. [CrossRef]

177. Musa, T.A.; Sanagi, M.M.; Ibrahim, W.A.W.; Ahmad, F.; Aboul-Enein, H.Y. Determination of 4-allyl resorcinol and chavibetol from piper betle leaves by subcritical water extraction combined with high-performance liquid chromatography. Food Anal. Methods 2013, 7, 893-901. [CrossRef]

178. Samadi, M.; Abidin, Z.Z.; Yoshida, H.; Yunus, R.; Biak, D.R.A.; Lee, C.H.; Lok, E.H. Subcritical water extraction of essential oil from Aquilaria malaccensis leaves. Sep. Sci. Technol. 2019, 55, 2779-2798. [CrossRef]

179. Fernández-Pérez, V.; Jiménez-Carmona, M.M. An approach to the static-dynamic subcritical water extraction of laurel essential oil: Co Mparison with conventional techniques. Analyst 2000, 125, 481-485. [CrossRef]

180. Halim, N.A.A.; Abidin, Z.Z.; Siajam, S.I.; Hean, C.G.; Harun, M.R. Screening of factors influencing the yield of Citrus hystrix leaves essential oil extracted via pressurized hot water extraction based on resolution $\mathrm{V}$ fractional factorial design. J. Food Eng. 2020, 43, 1-13. [CrossRef]

181. Pavlić, B.; Vidović, S.; Vladić, J.; Radosavljević, R.; Zeković, Z. Isolation of coriander (Coriandrum sativum L.) essential oil by green extractions versus traditional techniques. J. Supercrit. Fluids 2015, 99, $23-28$.

182. Filly, A.; Fabiano-Tixier, A.S.; Louis, C.; Fernandez, X.; Chemat, F. Water as a green solvent combined with different techniques for extraction of essential oil from lavender flowers. Comptes Rendus Chim. 2016, 19, 707-717. [CrossRef]

183. Liu, F.; Wan, S.Y.; Jiang, Z.; Li, S.F.; Ong, E.S.; Osorio, J.C. Determination of pyrrolizidine alkaloids in comfrey by liquid chromatography-electrospray ionization mass spectrometry. Talanta 2009, 80, 916-923. [CrossRef]

184. Mokgadi, J.; Turner, C.; Torto, N. Pressurized hot water extraction of alkaloids in goldenseal. Am. J. Anal. Chem. 2013, 4, 398-403. [CrossRef]

185. Jokic, S.; Gagic, T.; Knez, Z.; Subaric, D.; Skerget, M. Separation of active compounds from food by-product (Cocoa shell) using subcritical water extraction. Molecules 2018, 23, 1408. [CrossRef]

186. Šeremet, D.; Durgo, K.; Jokić, S.; Huđek, A.; Cebin, A.V.; Mandura, A.; Jurasović, J.; Komes, D. Valorization of banana and red beetroot peels: Determination of basic macrocomponent composition, application of novel extraction methodology and assessment of biological activity in vitro. Sustainability 2020, 12, 4539. [CrossRef]

187. Narita, Y.; Inouye, K. High antioxidant activity of coffee silverskin extracts obtained by the treatment of coffee silverskin with subcritical water. Food Chem. 2012, 135, 943-949. [CrossRef]

188. Machmudah, S.; Lestari, S.D.; Widiyastuti; Wahyudiono; Kanda, H.; Winardi, S.; Goto, M. Subcritical water extraction enhancement by adding deep eutectic solvent for extracting xanthone from mangosteen pericarps. J. Supercrit. Fluids 2018, 133, 615-624. [CrossRef]

189. Hashim, N.A.; Mudalip, S.K.A.; Harun, N.; Man, R.C.; Sulaiman, S.Z.; Arshad, Z.I.M.; Shaarani, S.M.; Azmir, J. Mahkota dewa Subcritical water extraction process: Experimental and molecular dynamics simulation study. Chem. Eng. Technol. 2019, 42, 1747-1756. [CrossRef]

190. Yang, R.; Huang, P.; Qiu, T. Ultrasound-enhanced subcritical water extraction of naphthoquinone pigments from purple gromwell (Lithospermum erythrorhizon) to higher yield and bioactivity. Food Sci. Biotechnol. 2013, 22, 671-676. [CrossRef]

191. Anekpankul, T.; Goto, M.; Sasaki, M.; Pavasant, P.; Shotipruk, A. Extraction of anti-cancer damnacanthal from root of Morinda citrifolia by subcritical water. Sep. Purif. Technol. 2007, 55, 343-349. [CrossRef] 
192. Shotipruk, A.; Kiatsongserm, J.; Pavasant, P.; Goto, M.; Sasaki, M. Pressurized hot water extraction of anthraquinones from the root of Morinda citrifolia. Biotechnol. Prog. 2004, 20, 1872-1875. [CrossRef] [PubMed]

193. Pongnaravane, B.; Goto, M.; Sasaki, M.; Anekpankul, T.; Pavasant, P.; Shotipruk, A. Extraction of anthraquinones from root of Morinda citrifolia by pressurized hot water: Antioxidant activity of extracts. J. Supercrit. Fluids 2006, 37, 390-396. [CrossRef]

194. Uzel, R.A.; Yaman, Ü.R. A novel method for food particle production using subcritical water extraction: Ganoderma mushroom as a case example. J. Supercrit. Fluids 2016, 111, 74-82. [CrossRef]

195. Ko, M.J.; Nam, H.H.; Chung, M.S. Subcritical water extraction of bioactive compounds from Orostachys japonicus A. Berger (Crassulaceae). Sci. Rep. 2020, 10, 10890. [CrossRef] [PubMed]

196. Liu, J.; Chen, P.; Yao, W.; Wang, J.; Wang, L.; Deng, L.; He, J.; Zhang, G.; Lei, J. Subcritical water extraction of betulinic acid from birch bark. Ind. Crop. Prod. 2015, 74, 557-565. [CrossRef]

197. Chi, X.; Zhang, G.; Chen, S. Subcritical water extraction of sesquiterpene lactones from Inula racemose. ChemistrySelect 2020, 5, 488-494. [CrossRef]

198. Cheng, Y.; Liu, W.; Gao, Q.; Yu, S.; Xue, F. Enhanced extraction of bioactive components of 3,29-dibenzoylkarounidiol and polysaccharides from Semen richonsanthis using subcritical water technology. ChemistrySelect 2019, 4, 13689-13694. [CrossRef]

199. Salami, A.; Asefi, N.; Kenari, R.E.; Gharekhani, M. Extraction of pumpkin peel extract using supercritical $\mathrm{CO}_{2}$ and subcritical water technology: Enhancing oxidative stability of canola oil. J. Food Sci. Technol. 2020, 54, 2306-2315. [CrossRef]

200. Falev, D.I.; Shestakov, S.L. Subcritical extraction of birch bark pentacyclic triterpenes. Russ. Chem. B 2017, 66, 875-881. [CrossRef]

201. Kovacevic, D.B.; Barba, F.J.; Granato, D.; Galanakis, C.M.; Herceg, Z.; Dragovic-Uzelac, V.; Putnik, P. Pressurized hot water extraction (PHWE) for the green recovery of bioactive compounds and steviol glycosides from Stevia rebaudiana Bertoni leaves. Food Chem. 2018, 254, 150-157. [CrossRef]

202. Wang, Y.; Zhang, G.; Chi, X.; Chen, S. Green and efficient extraction of podophyllotoxin from Sinopodophyllum hexandrum by optimized subcritical water extraction combined with macroporous resin enrichment. Ind. Crop. Prod. 2018, 121, 267-276. [CrossRef]

203. Gabaston, J.; Leborgne, C.; Valls, J.; Renouf, E.; Richard, T.; Waffo-Teguo, P.; Mérillon, J.M. Subcritical water extraction of stilbenes from grapevine by-products: A new green chemistry approach. Ind. Crop. Prod. 2018, 126, 272-279. [CrossRef]

204. Nile, S.H.; Nile, A.; Gansukh, E.; Baskar, V.; Kai, G. Subcritical water extraction of withanosides and withanolides from ashwagandha (Withania somnifera L) and their biological activities. Food Chem. Toxicol. 2019, 132, 110659. [CrossRef]

205. Najjar-Tabrizi, R.; Javadi, A.; Sharifan, A.; Chew, K.W.; Lay, C.H.; Show, P.L.; Jafarizadeh-Malmiri, H.; Berenjian, A. Hydrothermally extraction of saponin from Acanthophyllum glandulosum root-Physico-chemical characteristics and antibacterial activity evaluation. Biotechnol. Rep. 2020, 27, e00507. [CrossRef]

206. Güçlü-Üstündağ, Ö.; Balsevich, J.; Mazza, G. Pressurized low polarity water extraction of saponins from cow cockle seed. J. Food Eng. 2007, 80, 619-630. [CrossRef] 